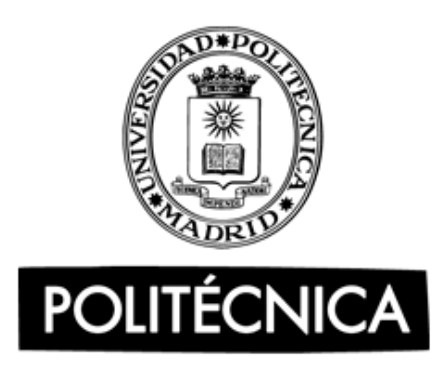

Universidad Politécnica de Madrid

ETSI Aeronáutica y del Espacio

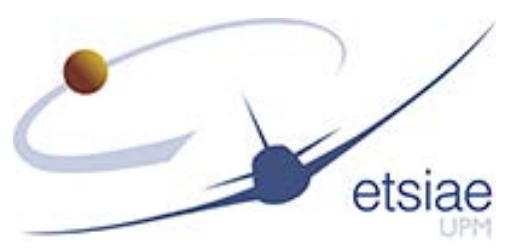

\title{
Single-axis Ultrasonic Anemometer in Rotational Motion
}

Doctoral Dissertation

Mohsen Ghaemi-Nasab

Madrid, 2018 



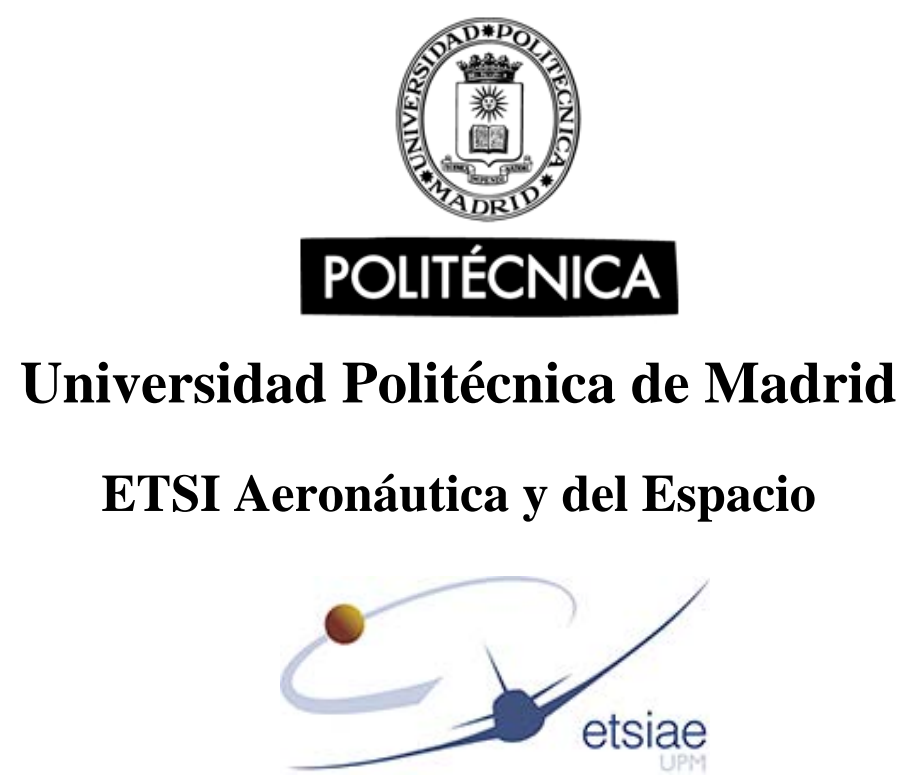

$\underline{\text { Doctoral Dissertation }}$

\section{Single-axis Ultrasonic Anemometer in Rotational Motion}

By:

Mohsen Ghaemi-Nasab

Advisors:

Dr. Sebastian Franchini

Dr. Felix Sorribes-Palmer

Madrid, March 2018 

Tribunal nombrado por el Sr. Rector Magfco. de la Universidad Politécnica de Madrid, el día................de..............................de 20....

Presidente:

Vocal:

Vocal:

Vocal:

Secretario:

Suplente:

Suplente:

Realizado el acto de defensa y lectura de la Tesis el día...........de........ de $20 \ldots .$.

en la E.T.S.I. /Facultad.................................................

Calificación

EL PRESIDENTE

LOS VOCALES

\section{EL SECRETARIO}





\section{Resumen}

Los anemómetros ultrasónicos son instrumentos modernos para la medida exacta de la velocidad del viento que se han utilizado extensamente en aplicaciones meteorológicas e industria de la energía eólica. A pesar de su extendida aplicación es cada vez mayor, ya se han prestado poca atención a los anemómetros ultrasónicos giratorios de eje único que pueden medir con precisión tanto la velocidad del viento como su dirección en un aparato único y robusto. Recientemente, se emplean en plataformas móviles tales como es el caso de los aerogeneradores. Esto requiere un proceso de calibración exacto y correcto para corregir las señales de salida del anemómetro durante la rotación. En esta tesis, se han realizado varias series de pruebas en túnel de viento para estudiar sus mediciones de velocidad en condiciones oscilatorias utilizando un anemómetro ultrasónico modificado de un eje giro.

Este estudio se ha llevado a cabo realizando medidas estáticas a diferentes ángulos de trayectoria de señal de los transductores con la dirección del viento, y con medidas dinámicas en las que el anemómetro está girando a diferentes velocidades de rotación. La velocidad medida por el anemómetro ultrasónico en los ensayos estáticos se calibra con datos de tubo pitot y la interpolación de los coeficientes de calibración obtenidos se utiliza para corregir la velocidad ultrasónica medida en ensayos dinámicos. Se observa que las mediciones de velocidad del viento con el anemómetro ultrasónico tras la calibración se ajustan adecuadamente a las velocidades de referencia en pruebas dinámicas. De acuerdo con los resultados, las mediciones de velocidad con el anemómetro ultrasónico en pruebas estáticas y dinámicas se ven afectadas por las distorsiones de la cabeza de los transductores y el desplazamiento en la trayectoria del pulso acústico debido al movimiento rotacional no afecta las mediciones del anemómetro en los casos dinámicos. Además, se obtiene un ángulo de fase en función de la orientación de la trayectoria de señal del anemómetro a partir de los ensayos estáticos que se debe al efecto de sombra del transductor y se utiliza para construir un modelo de calibración para corregir la velocidad del viento medida por anemómetro ultrasónico tanto estática como dinámicas.

Se observa que, en las pruebas dinámicas, el retardo o retraso entre el anemómetro ultrasónico y el sensor de posición angular junto con el efecto Doppler, imparten un desplazamiento de fase en las señales medidas por el anemómetro, que se debe añadir a los datos de posición para corregir el desalineamiento de guiñada. Los datos experimentales se utilizaron para construir un modelo teórico, basado en el Método de 
Superficie de Respuesta, para corregir el cambio de fase para varias velocidades del viento y velocidades de rotación del anemómetro. Este modelo ha demostrado corregir con éxito la velocidad indicada por el anemómetro giratorio para el cambio de fase debido a la rotación, y puede usarse fácilmente en el proceso de calibración para tales anemómetros.

Por otro lado, los datos experimentales han servido para validar un modelo teórico, basado en el Método de Superficie de Respuesta, y predecir la velocidad de salida del anemómetro para diferentes velocidades de viento y rotación como variables independientes. El modelo obtenido tiene una estructura simple y es fácil de usar, con una buena precisión para predecir los datos experimentales incluso en las zonas sombreadas del transductor. El modelo teórico propuesto puede usarse en la calibración de dichos anemómetros en condiciones de rotación y funciona para la condición estacionaria estableciendo que la velocidad angular en el modelo sea cero. El mismo modelo con diferentes coeficientes constantes aún se mantiene para cualquier otro anemómetro ultrasónico. 


\section{Abstract}

Ultrasonic anemometers are the modern instruments for accurate wind speed measurements that have been widely used in meteorological applications and wind energy industry. Despite their widespread and ever increasing applications, little attentions have already been paid to the spinning single-axis ultrasonic anemometers which can accurately measure both the wind speed and its direction in a single and robust apparatus. Recently, they are employed in dynamic spinning conditions on mobile platforms such as spinner anemometry on wind turbines. This necessitates an exact and proper calibration process to correct the anemometer output signals during the rotation. In this thesis, series of wind-tunnel tests have been performed on a modified single-axis ultrasonic anemometer to study its velocity measurements in oscillatory conditions.

This has been carried out with static measures at various stationary angles of transducers signal path with wind direction, and with dynamic measures in which the anemometer is rotating with various rotational speeds. The velocity measured by ultrasonic anemometer in static tests is calibrated with pitot-tube data, and the interpolation of obtained calibration coefficients is used to correct the ultrasonic velocity measured in dynamic tests. It is observed that the calibrated ultrasonic wind speed measurements in dynamic tests are in a good agreement with the reference velocities. According to the results, the ultrasonic velocity measurements in both static and dynamic tests are affected by transducers head distortions, and the shifting in acoustic pulse trajectory due to the rotational motion does not affect the anemometer outputs in dynamic cases. Besides, a phase angle as a function of the orientation of anemometer's signal path is obtained from the static tests which is due to transducer shadow effect and it is used to construct a calibration model for correcting the wind speed measured by ultrasonic anemometer in both static and dynamic conditions.

It is observed that in the spinning tests, the time lag or delay between ultrasonic anemometer and angular position sensor along with Doppler Effect, impart a phase shift in the signals measured by the anemometer, which should be added into the position data for correcting the yaw misalignment. The experimental data were used to construct a theoretical model, based on Response Surface Method, to correct the phase shift for various wind speeds and anemometer rotational velocities. This model has been shown to successfully correct the velocity indicated by the spinning anemometer for the phase 
shift due to the rotation, and can easily be used in the calibration process for such anemometers.

Additionally, the experimental data were used to construct a theoretical model, based on Response Surface Method, to predict the anemometer output velocity for various wind and rotational speeds as the independent variables. The obtained model has a simple structure and is easy to use, with a good accuracy to predict the experimental data even in the shadow zones of the transducer. The proposed theoretical model can be used in the calibration of such anemometers in rotational conditions and works for the stationary condition by setting the angular velocity in the model to be zero. The same model with different constant coefficients still holds for any other ultrasonic anemometers. 


\section{Acknowledgements}

I would like to acknowledge Universidad Politécnica de Madrid for providing the fund which allowed me to undertake this dissertation supervised by Dr. Sebastian Franchini who I must give him thanks and appreciations for his wisdom, support and encouragement during this research.

I would like to express my sincere thanks to Dr. Angel Sanz-Andres, the director of “Instituto Micrigravedad Ignacio Da Riva” (IDR/UPM), who granted me to be part of this research center.

All my work colleagues are also appreciated with particular mention to Dr. Felix Sorribes-Palmer for sharing his knowledge and experiences as the co-advisor of the thesis, Rafael and Enrique, for their expert advices on electrical design and data acquisition system, Carlos and Pedro for helping to make the mechanical structure at IDR's workshop.

Last but not least, I would like to thank to Dr. Alireza Davari whose hints have really improved the quality of this dissertation.

I am truly indebted and thankful to my parents, for their unconditional love and support who without their inspirations I would not have been able to complete this work. And very special thanks to my wife, Marjan, for her love and faithful support during this journey. 


\section{Contents}

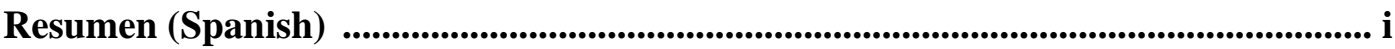

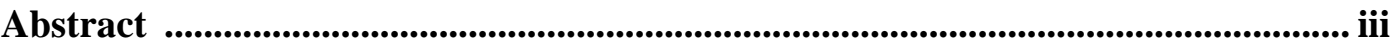

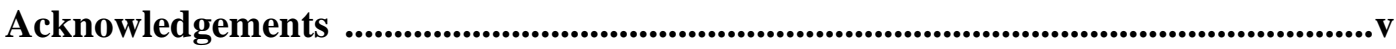

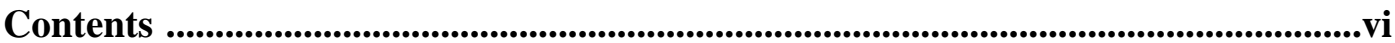

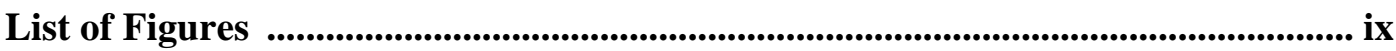

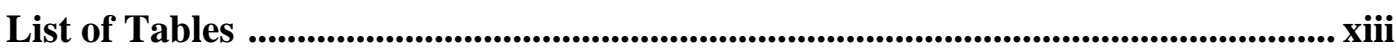

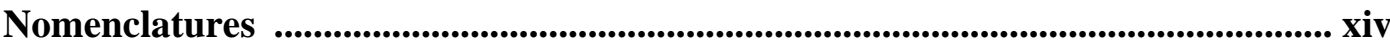

Chapter 1: Introduction ..............................................................................................................1

1.1. Chapters overview …................................................................................

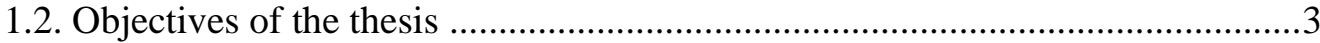

Chapter 2: Ultrasonic sensing technique and applications .........................................6

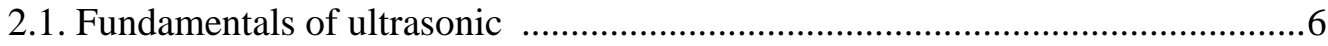

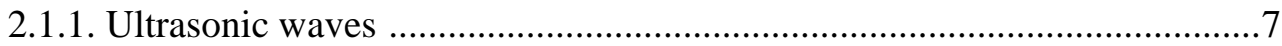

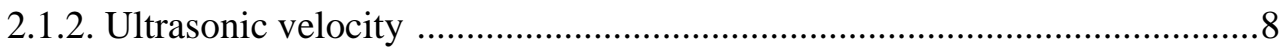

2.1.3. Ultrasonic generation and piezoelectric effect ........................................

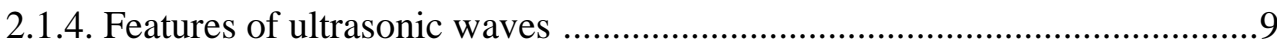

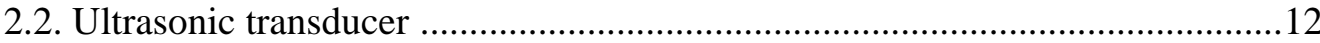

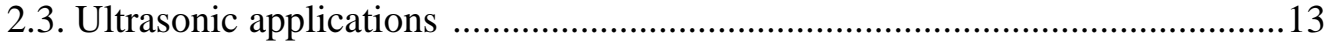

2.3.1. Ultrasonic flow metering …...............................................................14

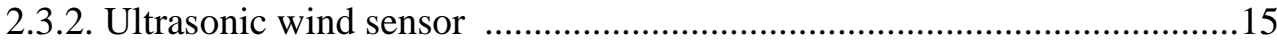

Chapter 3: Ultrasonic anemometer ............................................................................17

3.1. Ultrasonic transducer shadow effect ...........................................................19

3.2. Ultrasonic trajectory in rotational motion ...................................................21

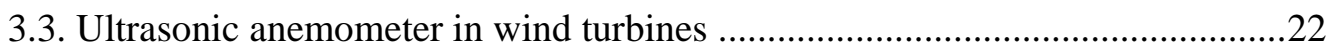

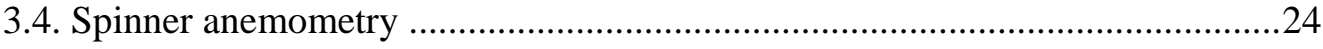

3.5. Calibration of spinner anemometry ……......................................................29

Chapter 4: K-style probe ultrasonic anemometer .....................................................33

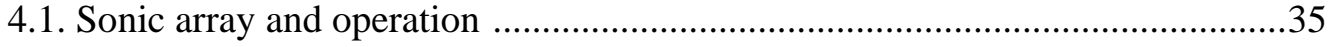

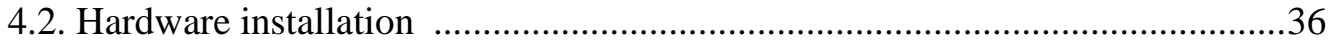

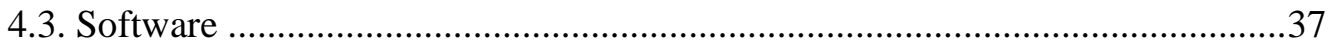




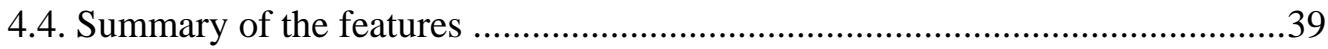

Chapter 5: Experimental setup and system design ......................................................40

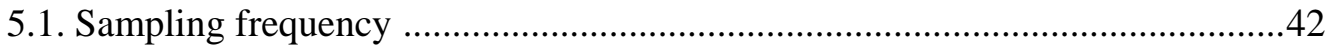

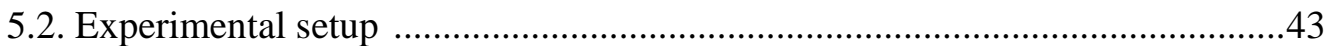

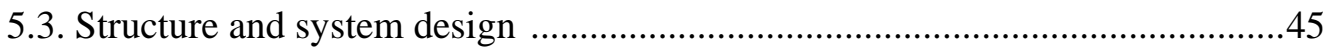

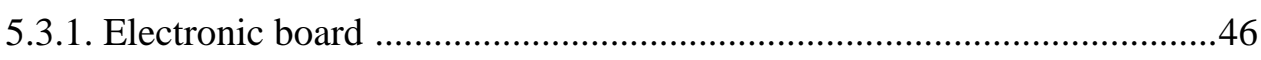

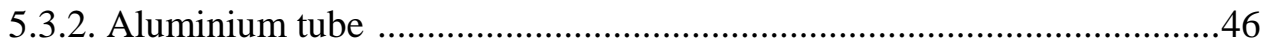

5.3.3. 3D upper connection ........................................................................47

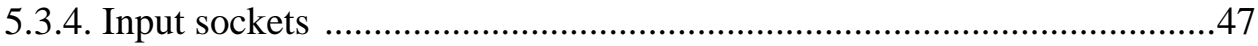

5.3.5. Sponge ..........................................................................................48

5.3.6. Slip-Ring-Capsule ..........................................................................48

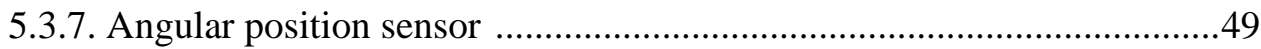

5.3.8. SCB-68 National instrument device ..................................................... 51

5.3.9. DC motor and controller ........................................................................51

5.3.10. Rectangular plate for holding the aluminium tube ..............................52

5.3.11. Triangular plate for holding the aluminium tube ................................52

5.3.12. Beams connection for structural rigidity ...........................................53

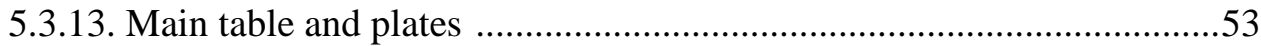

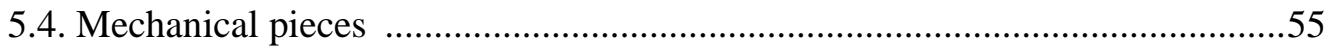

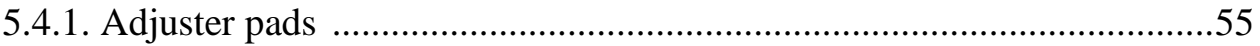

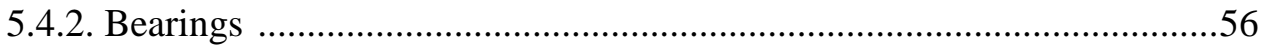

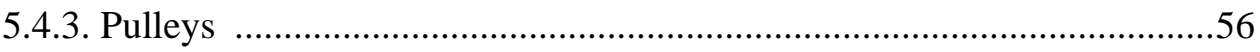

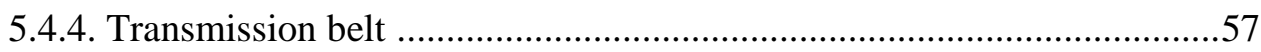

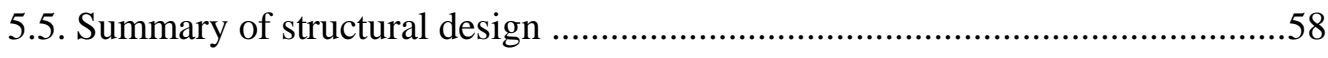

Chapter 6: Experiments and results ............................................................................60

6.1. Methodology and calibration process ........................................................60

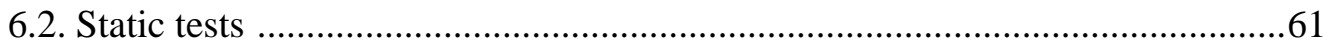

6.2.1. Calibration for static tests ...............................................................65

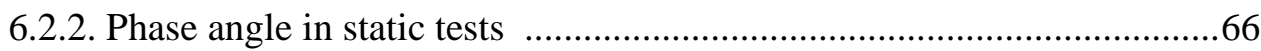

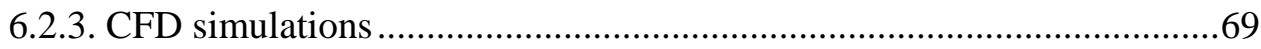

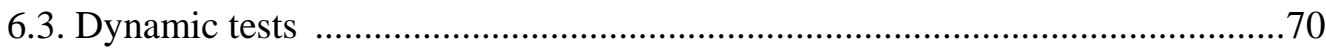

6.3.1. Accuracy of measurements ...............................................................71

6.3.2. Phase shift in dynamic tests .......................................................... 78

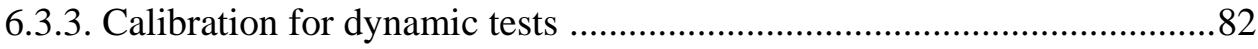




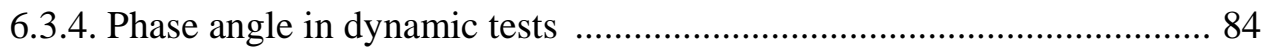

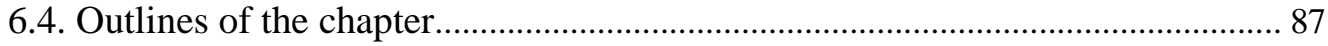

Chapter 7: Response Surface Methodology (RSM) ....................................................... 88

7.1. RSM to calibrate UA for yaw misalignment ....................................................... 91

7.2. RSM to calibrate UA for wind speed measurements ........................................ 99

7.3. Outlines of the chapter................................................................................. 105

Chapter 8: Conclusions and future work ......................................................................106

8.1. Calibration with least square regression …..................................................107

8.2. Calibration with phase angle ....................................................................... 108

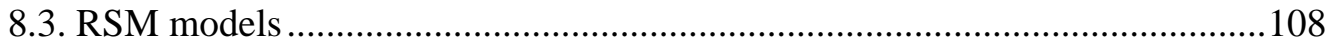

8.4. Calibration of UA in spinner anemometry …..............................................110

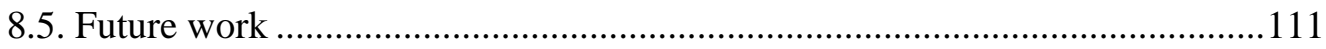

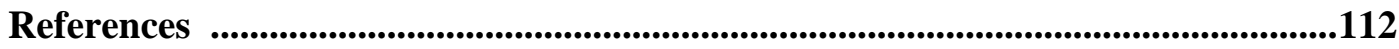

Appendix A: Specifications of belt and pulley ............................................................117

A.1. Belt and Pulley Pitch ..............................................................................117

A.2. Belt Length and Centre Distance ............................................................118

Appendix B: CFD simulations with Ansys-Fluent .....................................................120

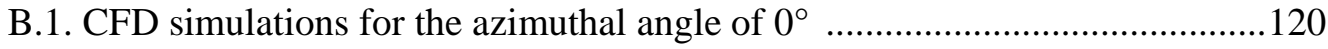

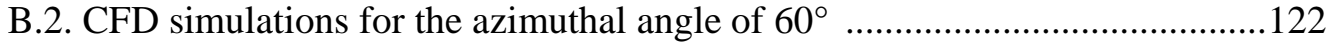

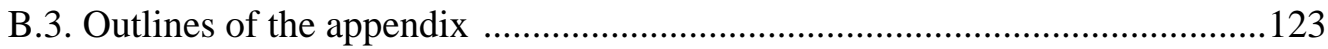




\section{List of Figures}

Figure 1.1: Ultrasonic anemometer used in spinner anemometry (right) and schematic view of a wind turbine spinner equipped with the spinner anemometry (left).................4

Figure 2.1: Normal reflection and transmission at an interface between two media ....11 Figure 2.2: Schematics definitions of wave reflection, refraction and mode conversion at an oblique interface

Figure 3.1: Basic configuration of an ultrasonic anemometer where $L$ is the acoustic path length, $D$ is the transducers diameter, $U_{\infty}$ is the uniform flow, and $c$ is the sound speed .18

Figure 3.2: Attenuation of flow speed along a single sonic path caused by the transducer shadow effect for BAO-type transducers (left graph) [53], and Kaijo-Denki type transducers (right graph) [55]

Figure 3.3: Top view of the one-axis UA, indicating: acoustic path length $(L)$, angle of attack between signal path and inflow direction ( $\theta$, azimuth angle), $U_{s}$ is the speed measured by UA along the signal path, and $U_{r}$ is the projection of inflow speed vector onto the transmitter-receiver axis.

Figure 3.4: Arrangement of wind sensing instruments on a fixed arm extending from the spinner of a wind turbine .24

Figure 3.5: A wind turbine hub equipped with the spinner anemometry; Top view of the wind turbine indicating the yaw misalignment $(\Gamma)$ in the horizontal plane of the spinner (a), and Side view of the wind turbine indicating the flow inclination angle $(\beta)$ in the vertical plane of the spinner (b) .25

Figure 3.6: Theoretical streamlines of the flowing around a sphere indicating the wind speed direction and tangential velocity at the point $(P)$ .26

Figure 3.7: Schematic view of a spinner anemometry indicating the spinner azimuthal position, $\varphi$, of flow stagnation point, and inflow angle, $\Theta$, in the horizontal and vertical plane of the wind turbine spinner with point $(P)$ in the middle of the acoustic measurement path which are identified as $\Theta_{h}$ and $\Theta_{v}$ respectively.... 27

Figure 4.1: K-style probe single-axis ultrasonic anemometer .34

Figure 5.1: Sketch of the S4 wind tunnel at the IDR/UPM: 1 = fans; 2 = plenum chamber; 3 = honeycomb and grids; 4 = contraction; 5 = test chamber; and $6=$ diffuser . .41 
Figure 5.2: Left: schematic side view of the experimental setup. Right: plan view, definition of ultrasonic path orientation, azimuthal angle, $\theta$, and the direction of the velocity vector measured by the ultrasonic anemometer, $U_{s}$

Figure 5.3: UA is installed in the test section of S4 wind-tunnel ready to be tested......45

Figure 5.4: Electronic Board of the tested UA .......................................................46

Figure 5.5: Aluminum Tube (Right) and its scheme draw (Left) .............................47

Figure 5.6: 3D upper connection made by injection printer.......................................47

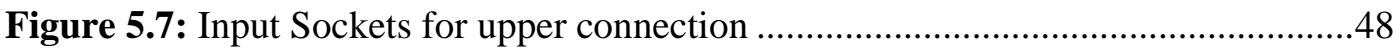

Figure 5.8: Sponge rolled around the electronic board .............................................48

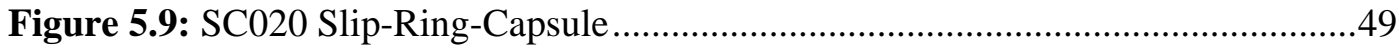

Figure 5.10: NRH280DP angular position sensor .....................................................49

Figure 5.11: Coupled system between motor's shaft and aluminum tube indicating the position of the angular position sensor and its magnetic bolt ..................................... 50

Figure 5.12: Output voltage of NRH280DP sensor based on the angle of rotation on

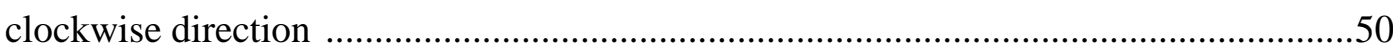

Figure 5.13: SCB-68 National Instrument data acquisition device .............................51

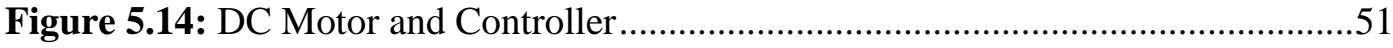

Figure 5.15: Rectangular flat plate for holding the aluminum tube .............................52

Figure 5.16: Triangular flat plate for holding the aluminum tube................................52

Figure 5.17: Beams used to connect the rectangualr and triangualr plates ...................53

Figure 5.18: Table to hold the DC-motor, angular sensor and slip-ring-capsule ...........53

Figure 5.19: Flat plate for holding the DC-motor ...................................................54

Figure 5.20: Flat plate for holding the angular position sensor.....................................54

Figure 5.21: Flat plate for holding the slip-ring-capsule ............................................55

Figure 5.22: Non-slip adjuster pads for the main table ...............................................55

Figure 5.23: Bearings for holding the aluminum tube ...........................................56

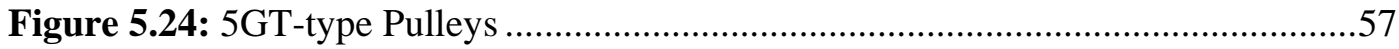

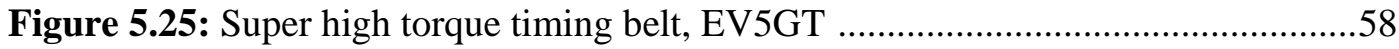

Figure 5.26: Schematic draw of the whole system with detail dimensions .................59

Figure 6.1: Wind speed measured by the UA based on the free stream wind speeds for different stationary azimuthal angles.

Figure 6.2: Ultrasonic velocity measurements, $U_{s}$, as a function of the reference velocity, $U_{r}$, for different stationary azimuthal angles, $\theta$. 
Figure 6.3: Velocity deficit, $\Delta U$, as a function of the absolute value of the reference velocity, $\left|U_{r}\right|$

Figure 6.4: Mean velocity deficit obtained for each reference velocity as a function of the azimuthal angle of the ultrasonic path, $\theta$.

.64

Figure 6.5: Standard deviation of the ultrasonic velocity measurements, $\sigma$, as a function of the free wind speeds, $U_{\infty}$.

Figure 6.6: UA measurements and the reference velocity (left), and the performance of the calibrated model (right). .66

Figure 6.7: Phase angle, $\phi$, as a function of the tested azimuthal angle, $0<\theta^{\circ}<180 \ldots \ldots . .67$

Figure 6.8: Phase angle, $\phi$, as a function of the azimuthal angle, $0<\theta^{\circ}<360$...............68

Figure 6.9: Entire experimental tests in which every circle indicates a dynamic test....70

Figure 6.10: Effects of the free stream wind speed on variations of the UA measured velocity .71

Figure 6.11: Effects of the UA rotational speed on variations of the UA measured velocity .71

Figure 6.12: UA indicated velocity at different instantaneous azimuthal angles for 10 revolutions .72

Figure 6.13: Histogram of the UA velocity measurements during 10 revolutions ........73

Figure 6.14: Histogram of the velocity measured by the spinning UA for two cycles of a typical test $\left(U_{\infty}=15.10 \mathrm{~m} / \mathrm{s}\right.$ and $\left.\omega=8.53 \mathrm{rad} / \mathrm{s}\right)$ : (a) the $15^{\text {th }}$ revolution, (b) the $35^{\text {th }}$ revolution... .75

Figure 6.15: Uncertainty measurement for a typical case ........................................76

Figure 6.16: Velocity deficit for a typical spinning test ..........................................77

Figure 6.17: Indication of the free stream wind and the instantaneous azimuthal angle for the spinner UA 79

Figure 6.18: Raw data of the velocities measured by UA for various incoming wind speeds at (a) low rotational speed, (b) moderate rotational speed, and (c) high rotational speed. And (d) non-dimensional velocity measurements for various UA rotational speeds

Figure 6.19: Variation of phase shift for various UA rotational speeds (Left), and for various free stream wind speeds (Right) .81

Figure 6.20: Corrected data for yaw misalignment of the velocities measured by UA for various incoming wind speeds at a rotational speed. 
Figure 6.21: Comparison of ultrasonic velocity measurements with reference velocities (a), and calibrated velocity measurements with reference velocities (b) .83

Figure 6.22: Regression coefficient, $R^{2}$, for the fitting of the calibrated measurements, $U_{c}(t)$ with the reference velocities, $U_{r}(t)$ as a function of the rotating velocities (Left) and of the wind-tunnel flow velocities (Right) .84

Figure 6.23: UA measurements and reference velocity (left), and calibrated velocity measurements and reference velocity (right) for a typical spinning test........................85

Figure 6.24: RMSE for variation of the wind speed and UA angular speed..................86

Figure 7.1: Response surface of the phase shift based on the independent variables ....93

Figure 7.2: Normal probability distribution of Eq. (7.3) ............................................94

Figure 7.3: Distribution of the residuals in Eq. (7.3) ................................................95

Figure 7.4: Corrected data for yaw misalignment of the velocities measured by UA for various incoming wind speeds at (a) low rotational speed and (b) high rotational speed

Figure 7.5: Response surface plot of the UA indicated velocity, $U_{s}$, as a function of the independent factors: azimuthal angles $\theta$, angular velocities $\omega$, wind speeds $U_{\infty}$

Figure 7.6: The normal probability of the residuals in Eq. (7.8) 101

Figure 7.7: The normal probability of the residuals in Eq. (7.9) 102

Figure 7.8: Performance of the present model obtained from RSM with present experiments for two typical spinning tests 104

Figure A.1: Pitch definition in belt and pulley 117

Figure A.2: Belt drive with unequal pulley diameters pulley 119

Figure B.1: UA model and meshing volumes for the azimuthal angle of $0^{\circ}$ 120

Figure B.2: Mesh grid of the test section and around the sonic transducers 121

Figure B.3: Contour of the velocity magnitude for a typical test with inlet velocity of $13.8 \mathrm{~m} / \mathrm{s}$ at the azimuthal angle of $0^{\circ}$ 121

Figure B.4: Contour of the velocity magnitude for a typical test with inlet velocity of $4.08 \mathrm{~m} / \mathrm{s}$ at the azimuthal angle of $0^{\circ}$. 122

Figure B.5: UA model and meshing volumes for the azimuthal angle of $60^{\circ}$ 122

Figure B.6: Contour of the velocity magnitude for a typical test with inlet velocity of $13.8 \mathrm{~m} / \mathrm{s}$ at the azimuthal angle of $60^{\circ}$ 123 


\section{List of Tables}

Table 1: General overview of the sequence steps necessary to calibrate a spinner anemometry 30

Table 2: Indication of which calibration steps should be performed depending on the intended use of the spinner anemometry 31

Table 3: Features of the K-style probe ultrasonic anemometer ...................................39

Table 4: List of the used equipment in the experiments ........................................... 41

Table 5: Configuration of sampling output in UA's serial port ................................. 42

Table 6: Tested rotational speeds of the UA sonic path ............................................... 44

Table 7: Principle configuration of the UA terminal setting ...................................... 44

Table 8: Calibration coefficients, $A$, and $B$, for different stationary azimuthal angles, $\theta$,

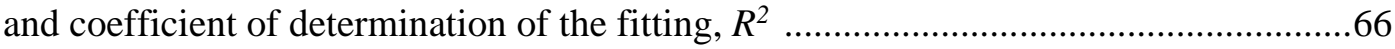

Table 9: Calculated statistical parameters for 10 revolutions of a typical test ...............73

Table 10: Statistical parameters of the indicated velocities during 7 revolutions for a

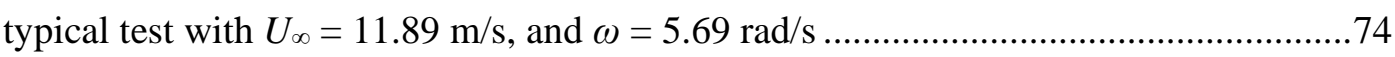

Table 11: Statistical data for a typical test with $U_{\infty}=16.73 \mathrm{~m} / \mathrm{s}$ and $\omega=3.18 \mathrm{rad} / \mathrm{s}$.........76

Table 12: Wind speeds and UA angular speeds of some typical dynamic cases ...........84

Table 13: P-values and VIF for each term in the regression mode ...............................96

Table 14: P-values for the regression terms in Eq. (7.8) ...........................................101

Table 15: P-values for the regression terms in Eq. (7.9) ............................................102 


\section{Nomenclatures}

\begin{tabular}{|c|c|c|}
\hline Value & Unit & Meaning \\
\hline$U A$ & - & Ultrasonic Anemometer \\
\hline$P_{i}$ & - & Ultrasonic transducers \\
\hline$c$ & $\mathrm{~m} / \mathrm{s}$ & Sound speed in the air \\
\hline$L$ & $\mathrm{~m}$ & Acoustic path length between transducers \\
\hline$D$ & $\mathrm{~m}$ & Diameter of the ultrasonic transducer \\
\hline$t_{+}$ & s & Transit time in forward direction \\
\hline$t-$ & $\mathrm{s}$ & Transit time in backward direction \\
\hline Z & s & Time delay between two consecutive pulses \\
\hline$d t$ & $\mathrm{~s}$ & Time step of the sampling records \\
\hline$F$ & $\mathrm{~Hz}$ & Sampling frequency \\
\hline$\omega$ & $\mathrm{rad} / \mathrm{s}$ & Angular velocity of the ultrasonic anemometer \\
\hline$U_{\infty}$ & $\mathrm{m} / \mathrm{s}$ & Free stream wind speed \\
\hline$U_{s}$ & $\mathrm{~m} / \mathrm{s}$ & Ultrasonic velocity measurement \\
\hline$U_{r}$ & $\mathrm{~m} / \mathrm{s}$ & Reference velocity \\
\hline$U_{c}$ & $\mathrm{~m} / \mathrm{s}$ & Calibrated velocity \\
\hline$U_{t h}$ & $\mathrm{~m} / \mathrm{s}$ & Theoretical model \\
\hline$\Delta U$ & $\mathrm{~m} / \mathrm{s}$ & Velocity deficit \\
\hline$\sigma$ & $\mathrm{m} / \mathrm{s}$ & Standard deviation \\
\hline$\sigma_{\text {pooled }}$ & $\mathrm{m} / \mathrm{s}$ & Pooled standard deviation \\
\hline$v$ & volt & Voltage output of the angular position sensor \\
\hline$\theta$ & rad & $\begin{array}{l}\text { Azimuthal angle between transducers' signal path } \\
\text { and wind direction }\end{array}$ \\
\hline$\phi$ & rad & Phase angle \\
\hline$\Delta \theta$ & deg & Phase shift in dynamic measurements \\
\hline$T$ & ${ }^{\circ} \mathrm{C}$ & Temperature in Celsius \\
\hline$T_{k}$ & ${ }^{\circ} \mathrm{K}$ & Temperature in Kelvin \\
\hline$\lambda$ & m/Hz.s & Acoustic wavelength \\
\hline$\rho$ & $\mathrm{Kg} / \mathrm{m}^{3}$ & Density \\
\hline$v$ & $\mathrm{~m} / \mathrm{s}$ & Particle velocity \\
\hline
\end{tabular}




\begin{tabular}{|c|c|c|}
\hline$\zeta$ & $\mathrm{N} / \mathrm{m}^{2}$ & Stress \\
\hline$I_{m}$ & $\mathrm{~N} . \mathrm{s} / \mathrm{m}^{3}$ & Acoustic impedance \\
\hline$K$ & $\mathrm{Kg} / \mathrm{m} \cdot \mathrm{s}^{2}$ & Bulk modulus \\
\hline$\gamma$ & - & Ratio of specific heat \\
\hline$R$ & $\mathrm{~J} / \mathrm{mole} . \mathrm{K}$ & Universal gas constant \\
\hline$M_{m}$ & $\mathrm{Kg} /$ mole & Molecular weight \\
\hline$A$ & - & Attenuation in acoustic waves \\
\hline$\alpha$ & - & Attenuation coefficient \\
\hline$f_{\text {sh }}$ & - & Shadow effect function \\
\hline$\delta, \zeta$ & - & Shadow effect parameters \\
\hline Uhor & $\mathrm{m} / \mathrm{s}$ & $\begin{array}{l}\text { Horizontal wind speed into the spinner } \\
\text { anemometry }\end{array}$ \\
\hline$U_{t}$ & $\mathrm{~m} / \mathrm{s}$ & Tangential airspeed around a sphere \\
\hline$\varphi$ & rad & Spinner azimuth position \\
\hline$\Theta_{h}$ & rad & Inflow horizontal angle to wind turbine spinner \\
\hline$\Theta_{v}$ & rad & Inflow vertical angle to wind turbine spinner \\
\hline$\theta_{p}$ & rad & Spinner anemometry pitching angle \\
\hline$\theta_{y}$ & rad & Spinner anemometry yawing angle \\
\hline$\beta$ & rad & Flow inclination angle in spinner anemometry \\
\hline$\Gamma$ & rad & Yaw misalignment in spinner anemometry \\
\hline$P_{w}$ & watt & Power of the wind turbine \\
\hline$d$ & $\mathrm{~m}$ & Diameter of the wind turbine blade \\
\hline
\end{tabular}




\section{Chapter 1}

\section{Introduction}

Ultrasonic sensors are widely used in variety fields of engineering and scientific applications. Recently, many types of conventional ultrasonic instruments, devices and sophisticated software are commercialized and used for both industrial and medical applications. One of the advantages of ultrasonic sensing is its outstanding capability to probe inside objectives nondestructively because ultrasound can propagate through any kinds of media including solids, liquids and gases except vacuum. In the typical ultrasonic sensing, the ultrasonic waves are travelling in a medium and often focused on evaluating objects so that a useful information on the interaction of ultrasonic energy with the objects are acquired as the ultrasonic signals which are the wave forms variations with transit time. Such ultrasonic data provides the fundamental basis for describing the outputs of ultrasonic sensing and evaluating systems.

In general, the ultrasonic sensors are based on measuring the properties of sound waves with frequency above the human audible range. They are based on three physical principles: time of flight, the Doppler Effect, and the attenuation of sound waves. Ultrasonic wind sensors measure flow speed by measuring the influences of flow field on the time of flight of the ultrasound pulses, which travel between pairs of transducers. In 
this dissertation, a deeply survey has been conducted on wind speed measurements with an ultrasonic anemometer in static and dynamic conditions.

Ultrasonic anemometer has been used in atmospheric flow studies since 1950s [1] and it has been known as a suitable instrument for measuring the turbulent flows [2]. These anemometers are extensively used in meteorological and pollution studies [3] and they are widely used in wind energy and civil aerodynamics applications. They are capable of measuring the wind velocity vector at one point, that drive the loading state of large structures e.g. wind turbines, bridges, buildings or their power performance in case of wind turbines [4]. Since their measurements are used for regulating the hub direction into the maximum free wind speed to optimize wind energy and power, the accuracy of their measurements are always be important for the wind energy sector.

\subsection{Chapters overview}

The body of this dissertation is organized as the following:

Chapter 1 consists of a brief introduction about ultrasonic sensor and objectives of the thesis. Chapter 2 presents the fundamentals of ultrasonic sensing technique and its applications in different areas of engineering. This chapter concentrated on the application of ultrasonic anemometers in wind energy specifically in wind turbines. The measurement principles of the ultrasonic anemometer and spinner anemometry are described in Chapter 3, and the advantages of spinner anemometry as a newly alternative technique for nacelle anemometry in wind turbines are highlighted in this chapter. The calibration process for wind speed and angular measurements in spinner anemometry is also explained.

Chapter 4 introduces the single-axis k-style probe ultrasonic anemometer used in the experiments including the descriptions of its mechanism and software for recording the measurements. Chapter 5 includes a complete review on the experimental setup, equipment and tools which are used in the experimental wind-tunnel tests.

In Chapter 6, the results from the experimental tests in static and dynamic conditions are presented, two calibration procedures for wind speed measured by ultrasonic anemometer along with the uncertainty analysis are presented. In Chapter 7, Response Surface Methodology (RSM) is used to obtain two prediction models for correcting the yaw misalignment and for predicting the wind speed measurements with the spinning ultrasonic anemometer. 
Finally, the conclusions of the thesis and the aspirations for future work about the calibration process and optimization of the prediction model are outlined in Chapter 8.

\subsection{Objectives of the thesis}

Ultrasonic anemometer has been used as a reliable and accurate instrument for measuring the wind speed and direction. Their operation system is not complicated and they provide sufficiently accurate wind speed measurements compared to other anemometers. Their advantages such as less maintenance and simultaneous measurement of wind speed and direction in one single instrument are highlighted for the wind turbine annual energy production. The fact is that the accurate wind speed and direction measurements in a wind turbine leads to a better regulation of the wind turbine spinner towards the optimum inflow direction, which consequently optimizes the wind power and increases the annual energy production. Therefore, the increasing in accuracy of wind speed and direction measurements and optimizing the measuring system with ultrasonic anemometers used in wind turbines are considered as an important investment for wind energy industry.

In a wind turbine, 2D ultrasonic anemometers are commonly used at the end of the nacelle chamber for measuring the wind speed which is known as the nacelle anemometry. Recently three 1D ultrasonic anemometers are used for measuring the wind speed and direction over the wind turbine spinner which is known as the spinner anemometry (iSpin technology), patented by Pedersen in 2008 [5].

Previous researches $[6,7]$ described problems related to cup anemometers and wind vanes, or 2D sonic anemometers, mounted on top of the nacelle. The flow distortions caused by the blades, nacelle chamber and its installations affect the nacelle anemometry measurements. This flow distortion is normally corrected in the wind turbine control system. However, corrections in the controller might not take all situations into account, for example, when the wake swirl from upstream wind turbines introduces upwards and downwards flow on the nacelle [8]. Such corrections are not required for a spinner anemometry which is not affected by those wakes since it measures the wind upstream of the rotor. Fig. 1.1 (right) shows a 1D sonic sensor used in spinner anemometry technology, and (left) illustrates a schematic view of a wind turbine equipped with the spinner anemometry. 

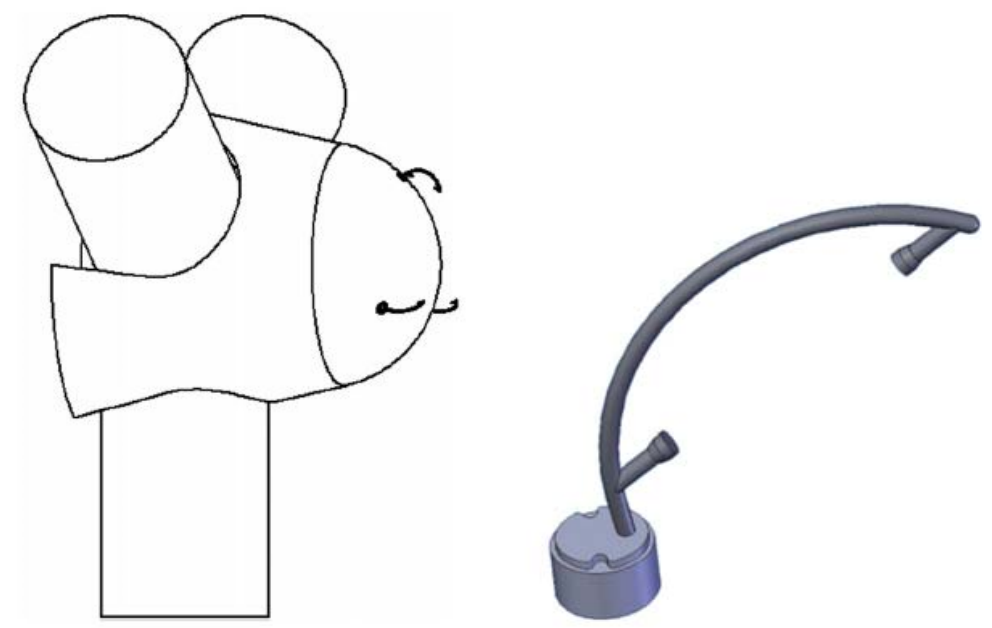

Fig. 1.1: Ultrasonic anemometer used in spinner anemometry (right) and schematic view of a wind turbine spinner equipped with the spinner anemometry (left).

The measurement principles of the spinner anemometry will be discussed in chapter 3. Here, briefly speaking, it is based on the flow over the spinner, which is measured close to the spinner surface with three single-axis ultrasonic wind sensors. The ultrasonic sensors use typically an acoustic path placed approximately $10 \mathrm{~cm}$ above the spinner surface. Accelerometers integrated in the sonic sensors are used to calculate the rotor position [5]. However, the sonic sensors are rotating with the wind turbine spinner during the operation and wind direction continuously changes, so a proper calibration process in order to provide the quality measurements of the wind speed and direction is a must.

In fact, a properly calibrated anemometer will provide good measurements of the wind speed which is extremely important for energy industry, as wind energy is proportional to the third power of the wind speed [9]. In a wind turbine, accurate knowledge of the incoming wind speed and direction is important in order to regulate spinner and blades for optimized wind turbine power. Investigations of the power performance losses [10] indicate that the power output of a wind turbine is reduced with a $\cos ^{2}$-function of the yaw error. A significant increase in wind turbine annual energy production has been realized by correcting the yaw errors as reported by Gorgen [11]. He claimed that the spinner anemometry is an accurate and reliable instrument for the measurement of yaw errors.

Therefore, an accurate wind speed measurement, as performed by a properly calibrated anemometer, would be extremely needed to achieve the maximum performance from energy saving viewpoint. 
Although there are investigations in which the performance of the spinner anemometry is evaluated on wind turbine, $[12,13]$, it has not found a systematic study on the behavior of these devices in rotational conditions. According to the Standard: IEC 61400-12-2 [14], the calibration process that uses for spinner anemometry consists of three major phases including the wind tunnel calibration tests for each ultrasonic sensor, the calibration of each spinner UA installations, and the calibration of each wind turbine type.

At present, the calibration process for each UA, the first phase, is performed in an accredited wind-tunnel for various stationary angles of attack of the signal path towards wind direction $[14,15]$. However, in the present thesis, the wind speed measurements with a 1D sonic sensor are calibrated in the spinning conditions while the wind direction continuously changed.

The objective of this thesis is to propose a calibration process for wind speed indicated by the spinning single-axis UA. The calibration process consists of two series of experiments in an accredited wind-tunnel. Firstly, the UA was placed in various stationary angles of attack toward the inflow direction, and its measurements were calibrated with pitot-tube. The calculated calibration coefficients were then used for correcting the wind speed measured by the same ultrasonic anemometer in rotational motion.

The other objective of this thesis is to propose a prediction model for calibration of wind speed measured by the spinning ultrasonic anemometer. Therefore, Response Surface Method (RSM) is used to obtain two regression models, for predicting the wind speed and for correcting the yaw misalignment, during the rotational motion of the anemometer's signal path. The RSM approach is proposed as a technique that would be used for calibration of any other ultrasonic sensor with different application such as spinner anemometry. 


\section{Chapter 2}

\section{Ultrasonic sensing technique and applications}

This chapter describes the fundamentals of ultrasonic sensing technology and their associated use in various ranges of sensors in industrial applications. What is ultrasonic, how to produce and capture ultrasonic, what kinds of methods and equipment can be used to measure ultrasonic, and what kinds of information can be obtained from ultrasonic measurements. These questions are addressed in the following sections and the answers to the questions are briefly explained from the viewpoint of industrial applications. Physical properties as well as the limitations of the piezoelectric devices used in the ultrasonic sensors are described. Particularly, the usage of ultrasonic sensors in flow metering and wind speed measurements will be discussed.

\subsection{Fundamentals of ultrasonic}

In this section, the fundamentals of ultrasonic waves and ultrasonic piezoelectric transducers are briefly described. The nature of the ultrasonic waves and their associated parameters such as ultrasonic velocity are summarized. 


\subsubsection{Ultrasonic waves}

Ultrasonic waves are similar to sound waves, where both travel through a medium. Ultrasonic waves consist of high-frequency sound waves that are inaudible to human beings. The frequency of the ultrasonic waves is normally above $20 \mathrm{kHz}[16,17]$. As the sound waves travel through the air, they produce vibration in the air particles which changes the density and pressure of the air particles along the direction of motion of the wave. If a sound wave is moving from left to right through air, particles of air will be displaced both rightward and leftward as the energy of the sound wave passes through it. If the source of the sound waves vibrates in a sinusoidal form, the pressure variations are also sinusoidal.

In a continuous medium, the behavior of ultrasonic waves is closely related to a balance between the forces of inertia and of elastic deformation. An ultrasonic wave moves at a velocity, the wave velocity, that is determined by the material properties and shape of the medium, and occasionally the frequency. The ultrasonic wave imparts motion to the material when it propagates. This is referred to as particle motion, to distinguish it from the wave motion. This particle motion is usually specified as a particle velocity $v$. It is noted in the ultrasonic measurements that the particle velocity is much smaller than wave velocity. Also, one can understand that no ultrasonic wave propagates in vacuum because there are no particles that can vibrate in vacuum.

The balance between inertia and elasticity develops into a linear relationship between stress $(\zeta)$ and particle velocity $(v): \zeta=I_{m} \times v$, the proportional factor $\left(I_{m}\right)$ is called the specific acoustic impedance of an ultrasonic wave [18, 19]:

$$
I_{m}=\frac{\zeta}{v}=\rho c
$$

where $\rho$ is the density $\left(\mathrm{kg} / \mathrm{m}^{3}\right)$, and $c$ is the wave velocity $(\mathrm{m} / \mathrm{s})$. The acoustic impedance, $I_{m}\left(\mathrm{Ns} / \mathrm{m}^{3}\right)$, characterizes the ability of a material to vibrate under an applied force and can be considered as the residence of the material to the passage of ultrasonic waves.

The concept of using the acoustic impedance plays an important role in determining of acoustic transmission and reflection at a boundary of two media having different material properties and therefore, the acoustic impedance is an important parameter in the designing of ultrasonic sensors and sensing systems. 


\subsubsection{Ultrasonic velocity}

Ultrasonic velocity is probably the most important and widely used parameter in ultrasonic sensing applications. The ultrasonic velocity is defined by the rate of change of particle displacement with respect to time. Ultrasonic waves can only be propagated in a material medium. Different characteristics of different materials will influence the velocity of the sound differently. The characteristics of the material medium have effects on the velocity and the attenuation of ultrasonic waves. The speed of sound waves in a medium depends on the compressibility and density of the medium [20]. If the medium is a liquid or a gas and has a bulk modulus $K$, and density $\rho$; the speed of sound waves $(c)$ in that medium or fluid is given by the following expression [21]:

$$
c=\sqrt{\frac{K}{\rho}}
$$

The speed of sound $(c)$ changes according to the temperature of the medium that it is propagated in. The speed of sound in atmosphere reaches $331.45 \mathrm{~m} / \mathrm{s}$ at $0{ }^{\circ} \mathrm{C}$. The air speed of sound in terms of temperature can be determined with the following equation:

$$
c_{\text {air }}=c_{0}+Q T
$$

where Cair is the speed $(\mathrm{m} / \mathrm{s})$ of the sound in air, $T$ is the air temperature in degree Celsius, and $Q$ is the rate at which the speed changes with respect to the temperature, which is approximately $0.607 \mathrm{~m} / \mathrm{s}$ at every change of $1{ }^{\circ} \mathrm{C}$ in temperature. Therefore, the speed of propagation of sound in the air (Cair) is $343 \mathrm{~m} / \mathrm{s}$, at $20{ }^{\circ} \mathrm{C}$ and 1 atmosphere pressure. Alternatively, the following expression may be used for any temperature and any gas, using the equation of state for gases, the speed of sound may be written as [22]:

$$
c_{g a s}=\sqrt{\frac{\gamma R T_{k}}{M_{m}}}
$$

where $T_{k}$ is the temperature in ${ }^{\circ} \mathrm{K}, R$ is the universal gas constant which has the value 8.314 J/mole.K, and $M_{m}$ is the molecular weight, which for air is $0.029 \mathrm{~kg} / \mathrm{mole}$. And the ratio of the specific heats, $\gamma$, for the air is 1.402 .

The acoustic impedance factor for the air is calculated as: $I_{m}=\rho \times c_{a i r}=1.293 \times 343=$ $443.5\left(\mathrm{Ns} / \mathrm{m}^{3}\right)$ which represents the resistance for propagation of the ultrasonic sound in the air at $20^{\circ} \mathrm{C}$. 


\subsubsection{Ultrasonic generation and piezoelectric effect}

The generation of ultrasonic waves is similar to the generation of an audible sound wave using a speaker. The diaphragm of the speaker is electronically driven to move back and forth, which produces low pressure and high pressure points in the air. For ultrasonic wave generation, the diaphragm needs to move back and forth at a much greater rate than for an audible sound wave. The frequency and amplitude of sound waves can be measured by measuring the fluctuations and the pressure difference in air particles propagating sound waves through air.

Since the ultrasonic waves are high-frequency waves, the sensitivity of a device to detect high-frequency waves plays an important role in ultrasonic wave detection. The piezoelectric effect can be used to detect as well as generate ultrasonic waves. Nowadays, most practical ultrasound sources are based on the piezoelectric principle of transconduction [23]. Piezoelectric sources have the advantage of simple construction and operation, which makes them suitable for a variety of applications.

A piezoelectric ultrasound generator consists of a layer of piezoelectric material with thin metal electrodes on both its sides. If an alternating electrical voltage is applied to these electrodes, the thickness of the layer will vary according to the variations of the electrical field [23], thus fluctuations in the air or a medium will be produced. Alternatively, the piezoelectric effect can be reversed to detect ultrasonic waves and to transform waves into an electrical signal.

\subsubsection{Features of ultrasonic waves}

This section discusses some characteristics and physical factors that influence the propagation of ultrasonic waves. To design the ultrasonic sensors and develop the ultrasonic sensing systems, it is important to understand the behavior and properties of ultrasonic wave in media. Hence the phenomena such as Propagation, Wavelength, Attenuation, Reflection, and Refraction of the ultrasonic wave during its propagation are briefly described here:

\section{- Propagation:}

Waves that propagate wholly inside an object, independent of its boundary and shape, are called bulk waves. Two types of bulk waves can exist in an isotropic medium: longitudinal and shear. The longitudinal waves can be defined on this basis as waves in which the particle motion is parallel to the direction of the wave propagation. The shear 
waves are defined as waves in which the particle motion is perpendicular to the direction of the propagation. Both waves can exist in solids because solids, unlike liquids and gasses, have rigidity that is a resistance to shear as well as compressive loads. However, the shear waves cannot exist in liquids and gasses because of no resistance to shear roads in such media [24].

\section{- Wavelength:}

Wavelength $\lambda$ is the distance over which one spatial cycle of the wave completes and it is obtained with the following expression:

$$
\lambda=U_{s} / f
$$

where $U_{s}$ is the ultrasonic velocity and $f$ is the frequency. Wavelength is a useful parameter in ultrasonic sensing and evaluations. In ultrasonic detection of a small object, the smallest size that can clearly be detected must be larger than half a wavelength at the operating frequency. If the critical size of an object to be detected is known, such prior information on size is helpful for selecting an appropriate frequency for measurements [24].

\section{- Attenuation:}

When an ultrasonic wave propagates through a medium, ultrasonic attenuation is caused by a loss of energy in the ultrasonic wave and other reasons. The attenuation can be seen as a reduction of amplitude of the wave. There are some factors affecting the amplitude and waveform of the ultrasonic wave, such as ultrasonic beam spreading, energy absorption, dispersion, nonlinearity, transmission at interfaces, scattering by inclusions and defects, Doppler-Effect and so on. To characterize the ultrasonic attenuation quantitatively, attenuation coefficient $\alpha$ is defined as follows:

$$
A=A_{o} e^{-\alpha x}
$$

where $A$ is the peak amplitude of the wave at propagation distance $x$, and $A_{o}$ is the initial peak amplitude. The attenuation coefficient $\alpha$ is experimentally determined from the variation of the peak amplitude with the propagation distance, and it can be given in decibel per meter $(\mathrm{dB} / \mathrm{m})$. In general, the attenuation coefficient highly depends on the frequency and varies widely for different media [25, 26]. 


\section{- Reflection and transmission:}

When an ultrasonic wave perpendicularly impinges on an interface between two media as shown in Fig. 2.1, a part of a wave is reflected back to the medium 1 and the remainder is transmitted to the medium 2. The reflection and transmission at interface play an important role in designing ultrasonic sensing systems and understanding experimental results with the ultrasonic systems [27].

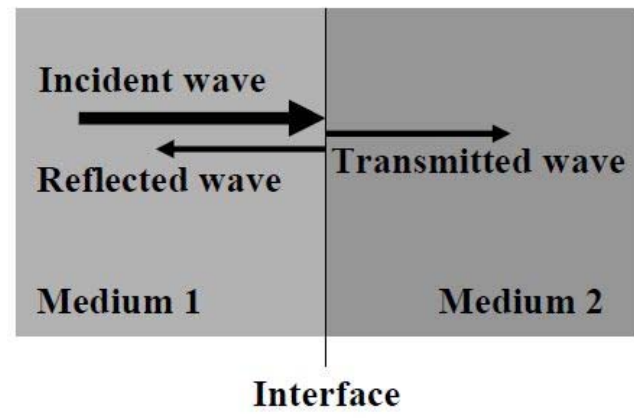

Fig. 2.1: Normal reflection and transmission at an interface between two media.

\section{- Refraction and mode conversion:}

When an ultrasonic wave obliquely impinges on an interface between two media as shown in Fig. 2.2, several things happen depending on the incident angle of the wave as well as the material properties of the two media. One of the important things is refraction in which a transmitted wave has a different angle from the incident. The refraction is basically caused by the velocity difference on either side of the interface. The refraction angle can be calculated from Snell's law [28] if the velocities of the two media and the incidence angle are known.

Another important phenomenon is mode conversion that is a generation of one type of wave from another type in refraction as shown in Fig. 2.2. For example, a longitudinal wave incident on an interface between liquid and solid is transmitted partially as a refracted longitudinal wave and partially as a mode converted shear wave in the solid. Mode conversion can also take place on reflection if the liquid shown in Fig. 2.2 is a solid. It is noted that any types of waves can be converted to another type, e.g. from a shear wave to a longitudinal wave, and from a longitudinal wave to a surface wave. The angles of reflection and/or refraction by mode conversion can be calculated from the Snell's law [28].

Further details on the features of ultrasonic wave are beyond the scope of this thesis and are left for the interested reader to find in other related literatures. 


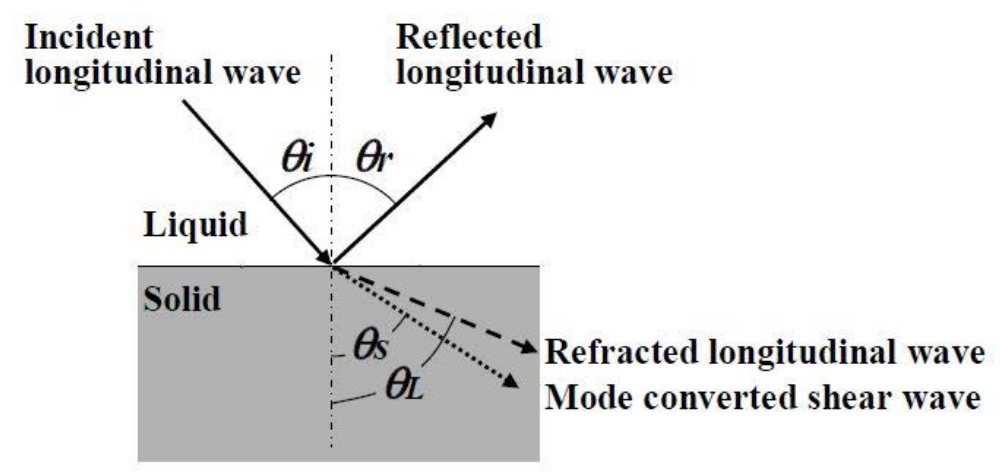

Fig. 2.2: Schematics definitions of wave reflection, refraction and mode conversion at an oblique interface.

\subsection{Ultrasonic transducer}

During ultrasonic wave generation, the transducer element i.e., piezoelectric is excited by applying an electrical signal across it. But during ultrasonic wave detection, an electrical voltage signal across the piezoelectric element is monitored. As soon as an ultrasonic wave strikes the transducer, the piezoelectric element vibrates accordingly; thus it generates a voltage signal across its terminals [26].

The function of the transducers is to convert electrical energy into mechanical energy which directly corresponds to ultrasonic vibration, and vice versa. The most common way of generating and detecting ultrasonic waves utilizes the piezoelectric effect of a certain crystalline material such as quartz. Since the piezoelectric effect is reciprocal, it produces a deformation (a mechanical stress) in a piezoelectric material when an electrical voltage is applied across the material, and conversely, it produces an electrical voltage when a deformation (a mechanical stress) is applied to the material. Thus, the piezoelectric materials can be used for generating and detecting ultrasonic waves that are related to the mechanical stresses. Appropriate cuts and directions of quartz are utilized for two types of waves, longitudinal and shear. The size and shape of piezoelectric transducers have to be precisely designed depending on the desired frequency. For industrial applications, solid-state transducers are usually used, because of their robustness. A piezoelectric transducer consists of a piezoelectric element, electrical connections, backing materials, front layers and a casing. The front layer is to protect the piezoelectric element against external stresses and environmental influences, and also must function as an impedance matching layer with which the transfer of ultrasonic energy to the target medium is optimized. The backing material functions as a damping block that alters the resonance 
frequency of the piezoelectric element and deletes unwanted ultrasonic waves reflected from the back wall. The electrical line is connected AC or DC voltage supplies that are often operated at the resonant frequency of the piezoelectric element [26].

\subsection{Ultrasonic applications}

Ultrasonic sensors are widely used in different field of engineering, industrial and medical which are described briefly in this section.

Ultrasonic sensors are used to detect the presence of targets and to measure the distance to targets in many automated factories and process plants. Ultrasonic sensors have gained popularity in a number of uses including ultrasonic people detection and assisting in autonomous UAV navigation. Because ultrasonic sensors use sound rather than light for detection, they work in applications where photoelectric sensors may not. Ultrasonic is a great solution for clear object detection, clear label detection and for liquid level measurement, applications that photoelectric struggle with because of target translucence. Target color and/or reflectivity don't affect ultrasonic sensors which can operate reliably in high-glare environments. A common ultrasonic application is an automatic door opener, where an ultrasonic sensor detects a person's approach and opens the door.

Ultrasonic cleaning is an environmentally friendly alternative for the cleaning of continuous materials, such as wire and cable, tape or tubes. The effect of the cavitation generated by the ultrasonic power removes lubrication residues like oil or grease, soaps, stearates or dust. In addition, the pollution particles are dispersed into the cleaning liquid. An ultrasonic transducer is affixed to a stainless steel pan which is filled with a solvent (frequently water or isopropanol) and a square wave is applied to it, imparting vibrational energy on the liquid. By that, a new adhesion to the material to be cleaned is avoided and the particles are flushed away [29].

Ultrasonic thickness measurement is one technique used to monitor quality of welds. Additionally, there is a need within the steel industry for accurate measurements of steel sheet thickness. This can be achieved by making ultrasonic pulse-echo measurements through the steel and calculating the time-of-flight of the ultrasonic. Since the velocity of the ultrasonic in the steel is known, the thickness of the sheet can thus be calculated. A further requirement of the steel industry is the assessment of the quality of the sheet steel. One of the flaws that often finds within steel is due to a phenomenon known as residual stress. This is caused by local deformations in the crystalline structure of the steel which 
occur during the cooling phase after hot rolling. Residual stresses within a structure cause local variations in the velocity of the ultrasonic, therefore a time-of-flight measurement which falls outside a given range may also be due to such flaws within the steel [30].

Medical ultrasonic transducers, probes, come in a variety of different shapes and sizes for use in making pictures of different parts of the body. Moreover, arterial diagnostic systems use ultrasonic to track blood flow throughout the body [31], and also the ultrasonic sensor is used for breath monitoring [32]. In the Doppler technique, the color flow mapping method is quite useful for detecting abnormal flow [33, 34].

Las but not least, ultrasonic sensors are widely used in the flow metering instruments and wind speed anemometers which are related to the area of research in this dissertation. Therefore, a brief description on these applications and backgrounds are described in the following subsections.

\subsubsection{Ultrasonic flow metering}

Since 1950s the ultrasonic flow measurement has been increased in the flow metering market [35, 36]. Ultrasonic flow meters offer the potential for accurate measurement of fluid volume over a wide flow range using a simple geometry by having very low maintenance because they do not use moving parts, unlike the mechanical flow meters.

By using the ultrasonic transducers, the flow meter can measure the average velocity along the path of an emitted beam of ultrasound, by averaging the difference in measured transit time between the pulses of ultrasound propagating into and against the direction of the flow or by measuring the frequency shift from the Doppler Effect. In general, the ultrasonic flow meters are affected by the acoustic properties of the fluid and can be impacted by temperature, density, viscosity and suspended particulates depending on the exact flow meter. An ideal ultrasonic flow meter would yield high accuracy reading independent of external influences, such as time, temperature, flow and installation conditions [37].

The ultrasonic flow meters have such advantages as follows: No pressure drop, High frequency pulse rate of output minimizes errors from effects of pulsation and fluctuating flow, Simple and inexpensive installation, High sensitivity, and No moving parts in contact with flowing fluid.

In addition, ultrasonic provides a key means to realize nonintrusive measurement for flow industry. The ultrasonic meter category contains a number of different designs for 
measuring an average velocity in a flowing system. They are all based on an ultrasonic signal being changed by or reflected from the flowing stream velocity.

The investigations on the ultrasonic flow metering technology has been developed by the Swengel in 1950s, such as his effort on the principles and applications of ultrasonic flow meter in the references [38, 39], till todays this instrument has evolved to support some of the most popular and efficient flow measurement devices.

The non-invasive nature of ultrasonic flow metering makes it highly applicable to oil and gas pipeline measurement systems, feed water flow measurement, as well as a plethora of other areas where other types of flow meters are not appropriate due to their intrusiveness or associated pressure loss [40].

Ultrasonic flow metering technologies have some of the more prominent benefits of ultrasonic systems include noninvasiveness, ease of operation and installation, fast response to flow variations, increased time between calibrations, and the modular design and interchangeability of components enabling rapid system repair. Not exclusive to the private sector, these ultrasonic devices can also be found in a domestic setting, utilized in hot water and methane metering systems used to monitor water and gas usage in large housing developments [40].

\subsubsection{Ultrasonic wind sensor}

Ultrasonic wind sensors have become the first choice for many applications in the field of outdoor measurements of the wind velocity vector. Ultrasonic anemometer is available for the measurement of airflow properties. This instrument now is robust and affordable to have more precise, higher frequency two- or three-dimensional measurement of flow speed and turbulence properties including quasi-instantaneous velocity fluctuations, Reynolds stress, and turbulence spectra [41].

According to wind measurement industry, cup anemometers do not measure properly the reference wind speed, since they deviate before some wind conditions such as high turbulence, and wind vertical components. Ultrasonic anemometers present a serious alternative to be assessed [42]. Ultrasonic anemometers are also quite known in the wind related research issues. They $3 \mathrm{D}$ types can measure the complete wind vectors $\mathrm{u}, \mathrm{v}, \mathrm{w}$ components [42].

Ultrasonic anemometers do not have any moving part, therefore they do not sensitive to dynamic effects, such as over speeding in cup anemometers. Also, since they are able 
to measure the three components of the wind, they permit to calculate the parameters such as Skewness and Kurtosis of wind speed, ratio of Standard Deviation of different wind vector components at the same point [43].

Sonic anemometers are commonly used in the areas of wind turbine control and at times wind resource assessment. In the application of turbine control, wind speed measurements from sonic sensors are used to validate the power output of the turbine and are also used for controlling the start-up and shut-down of a turbine. From years ago the 2D ultrasonic sensors are used in nacelle anemometry to measure the wind speed at the end of the nacelle. Recently, three 1D sonic sensors are placed over the wind turbine spinner to measure the wind speed and direction in front of the wind turbine. This newly developed technique is known as spinner anemometry and is broadly introduced and described in the next chapter of this thesis.

Another application of ultrasonic wind sensor is in aircraft industry including velocity and vortex measurement on the airplane's wing and in the airport terminal area in order to control the air traffic regarding the vortices generated by the planes after landing and take-off. Wake vortices pose a significant safety hazard to all air traffic and have been cited as the cause of many aircraft incidents and accidents [44]. These vortices trail from the wings of any aircraft producing lift, and may contain intense circulations that can cause structural damage or rolling of any aircraft that encounter them. Detecting these wake vortices is highly desirable, particularly in the vicinity of airports where aircraft are in close proximity and share common flight paths, making the probability of wake vortex encounters the greatest. A new ultrasonic method of vortex detection was deployed at an airport to detect wake vortex circulations from aircraft during low-pass flights over the airfield. Ultrasonic methods are utilized to set up inexpensive devices capable of directly measuring the circulation of vertical flows [45]. 


\section{Chapter 3}

\section{Ultrasonic anemometer}

Nowadays, the use of ultrasonic anemometer is increasing as a result of the growing importance of wind as a clean and renewable energy resource. Among the different types of anemometers that used to measure the wind speed, ultrasonic anemometers are the most common after considering all the possible applications. They are easy to operate without any moving parts, cost less to maintain and have longer life cycles than traditional cup and vane sensors, respond better to high turbulence and wind gusts, work well in freezing weather, and are able to measure wind speed and direction accurately in one single instrument.

For measuring wind velocity using an ultrasonic sensor, there are two methods: transit-time measurement and vortex measurement. In the vortex measurement method is difficult to measure the wind direction. So the method of transit-time measurement is used to measure the wind velocity and direction $[46,47]$. Since the ultrasonic anemometer used for the experiments in this thesis is based on the transit-time measurements, this literature is concentrated on transit-time method.

In the ultrasonic transit-time measurement method, the anemometer measures the wind speed in one point by detecting the influence of the wind speed field in the transmission of ultrasonic pulses along one or more acoustic paths configured by at least 
one pair of transmitter-receiver transducers. An ultrasonic anemometer measures the times taken for an ultrasonic pulse of sound to travel from an upstream transducer to the opposite downstream transducer, and compares it with the time for a pulse to travel from downstream to upstream transducer.

Each transducer emits an ultrasonic signal that travels towards the opposite transducer. The speed of propagation of the signals is the vector sum of the speed of sound in the media, $c$, and the local flow velocity vector along the measurement path. Therefore, the flow velocity field has a different effect on the propagation of signals in each direction. From the measurement of the differences between the forward and backward transit times, the wind speed component along the measurement path can be obtained [48]. The basic configuration of an ultrasonic anemometer is illustrated schematically in the Fig. 3.1.

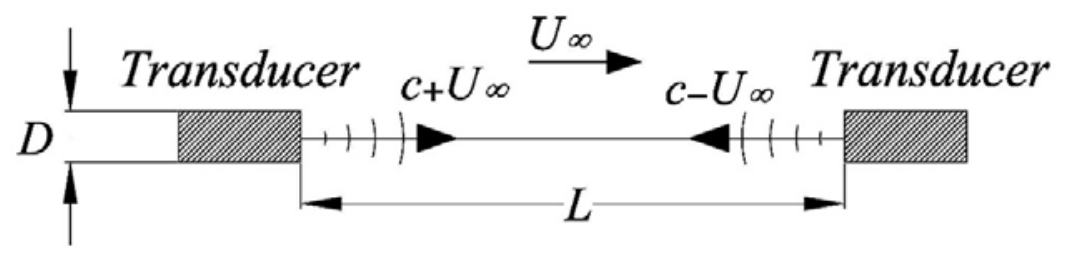

Fig. 3.1: Basic configuration of an ultrasonic anemometer where $L$ is the acoustic path length, $D$ is the transducers diameter, $U_{\infty}$ is the uniform flow, and $c$ is the sound speed.

If the measurement path of a sonic anemometer is modeled by the geometrical arrangement shown in the Fig. 3.1, the transit times in each direction could be related with the flow speed $\left(U_{\infty}\right)$, and the speed of sound $(c)$ which can be given as:

$$
t_{+}=\frac{L}{c+U_{\infty}} \quad ; \quad t_{-}=\frac{L}{c-U_{\infty}}
$$

where the + subscript refers to the transit time of the signal travelling from transmitter to receiver and the - subscript to the signal travelling in the opposite direction. The anemometer measures both transit times and determines the flow velocity using the following expression deduced from Eq. (3.1):

$$
U_{\infty}=\frac{L}{2}\left(\frac{1}{t_{+}}-\frac{1}{t_{-}}\right)
$$

This is the algorithm being used by most ultrasonic anemometers, regardless of the flow velocity field where $L$ is the length of the acoustic path, and $t_{ \pm}$is the time required by the pulse to cover the distance between transducers in sense of forward $(+)$ and 
backward (-) directions [49]. The measured flow velocity, as given by the anemometer, is obtained by applying the time inverse difference algorithm, Eq. (3.2).

\subsection{Ultrasonic transducer shadow effect}

A well-known phenomenon in sonic anemometry is the transducer shadow effect, the underestimation of the wind components due to velocity deficits in the wakes of the transducers along the acoustic paths [2]. This is the main source of uncertainty in the UA wind speed measurements which has been widely investigated [50, 51, 52, 53], and it has been shown that the wakes generated by the sensor head are variable for different anemometer geometries.

Wyngaard and Zhang [53] suggested that the dependence of the velocity attenuation on inclination angle $(\theta)$ is more sensitive to the design of the transducer than its dependence on $L / D$. $L$ is the distance between two transducers and $D$ is the diameter of transducers. They presented calculations for two varieties of transducers: right circular cylinders which they called BAO type; and streamlined transducers which they called Kaijo-denki type. They have suggested two analytical models for those two type of transducers of their directional response:

$$
\begin{gathered}
\frac{U_{t h}}{U_{r}}=f_{s h}=\delta+(1+\delta) \times \sin \theta \quad \text { (BAO-type) } \\
\frac{U_{t h}}{U_{r}}=f_{s h}=1-(1-\delta) \times \exp \left(-\zeta \times \sin ^{2} \theta\right) \quad \text { (Kaijo-Denki type) }
\end{gathered}
$$

$f_{s h}$ is the shadow effect correction function, $U_{t h}$, is the speed measured along the signal path and $U_{r}$ is the projection of the flow vector onto that path which is called as Reference Velocity in this thesis. The constants $\delta$ and $\zeta$ are array-specifics corresponding to a given ratio of $L / D$. In each case, $\delta$ is the response at $\theta=0$ which can be obtained from Fig. 3.2 where the left graph is for BAO-type transducers and the right graph is for Kaijo-Denki type transducers. $\zeta$ should be found from the best fitting of theoretical model, $U_{t h}$, with experimental data [54] (in reality a mathematical matching based in the calculation of $\delta$ and $\zeta$ by minimizing the rms error between experimental and theoretical model leads to obtain $\delta$ and $\zeta$. 


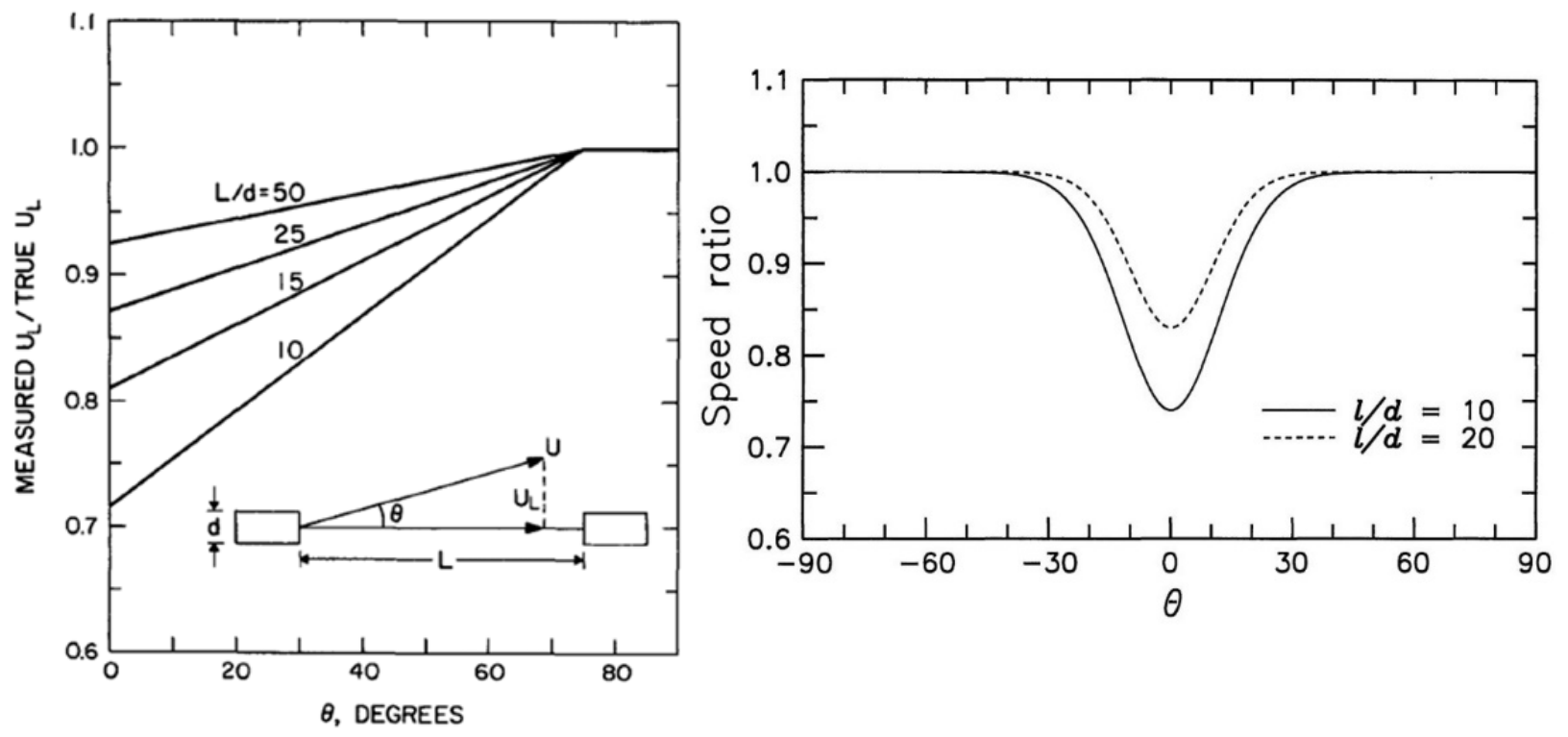

Fig. 3.2: Attenuation of flow speed along a single sonic path caused by the transducer shadow effect for BAO-type transducers (left graph) [53], and Kaijo-Denki type transducers (right graph) [55].

It is noted that the transducers used in the present experimental work, are the KaijoDenki type. Fig. 3.3 schematically presents the basic configuration of the single-axis UA with the definition of the azimuthal angle, $\theta$, between signal path and free stream wind direction.

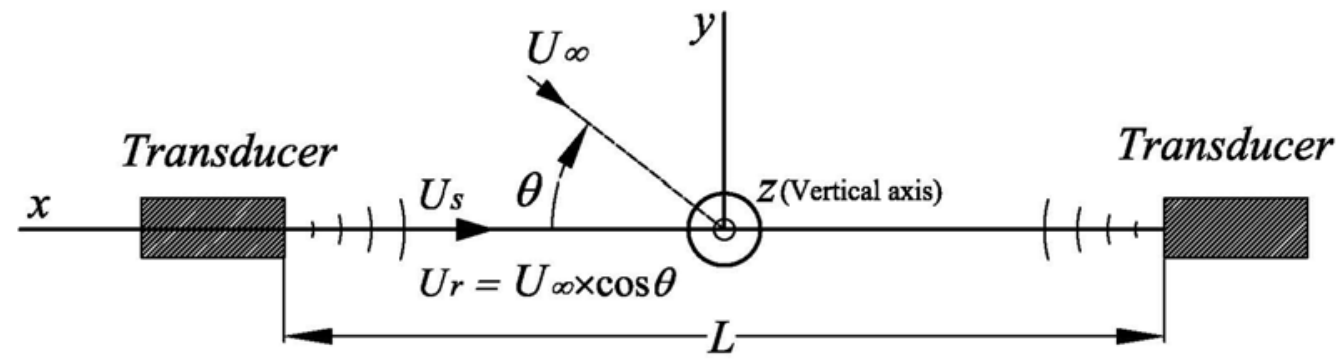

Fig. 3.3: Top view of the one-axis UA, indicating: acoustic path length $(L)$, angle of attack between signal path and inflow direction ( $\theta$, azimuthal angle), $U_{s}$ is the speed measured by UA along the signal path, and $U_{r}$ is the projection of inflow speed vector onto the transducers' axis. 


\subsection{Ultrasonic trajectory in rotational motion}

Franchini et al [48], developed a mathematical model of the measurement process performed by an ultrasonic anemometer, based on the acoustic geometric theory in order to determine the flow velocity component along the measurement path. The main contribution of their work was the consideration of the impact of the shift of the acoustic pulse trajectory (regarding to the straight line) on the speed measurement with ultrasonic anemometer. An approximate solution of the problem, the velocity measured by the anemometer, has been obtained by asymptotic techniques, based on the small value of the Mach number and the shift of the trajectory. Their concerns had not been taken into account in the previous published works where just the straight acoustic measurement path was considered [56, 57, 58, 59].

The mathematical model allowed Franchini et al. [48] to obtain the expressions of transit time in both directions as a function of the velocity field and its derivatives. Therefore, the problem consists of determination the minimum transit time trajectory between the transducers. Thus, based on the geometrical acoustic and variation method, forward and backward trajectories are determined and used for calculating the transit times in both directions. The "measured" speed (the speed measured by the sonic anemometer) was obtained and compared to the "true" speed (the average velocity along the measurement path). The "measured" speed contains two factors: (1) the average speed or "true" speed, and (2) a correction term which composed two terms; first: the contribution of the velocity field along the straight line which only depends on the uniformity of the velocity components (it is zero if the flow is uniform), second: the contribution of the trajectory shift effects including the variation along the measurement path of the value of the velocity components and their longitudinal and transversal derivatives.

The rotational motion of single-axis ultrasonic anemometer around its vertical axis makes the acoustic pulse trajectory is shifted with regard to the straight line. Franchini et al. [48] analyzed analytically the impact of the shifting in acoustic pulse trajectory (with regard to the transmitter-receiver straight line) caused by the velocity field on the $1 \mathrm{D}$ ultrasonic wind speed measurements. Due to their mathematical model, for the 1D vertical shear flow, both pulse trajectories are symmetric with regard to the transmitterreceiver axis so that the transit time in forward and backward directions are influenced in the same way; Indeed, the transit times of the pulses are both larger than in the case of 
unperturbed flow (straight uniform flow), and hence the UA measured speed is not affected by the shifting in acoustic pulse trajectories. Similarly, in the rotational motion of single-axis UA around its vertical-axis which is performed in the present experimental work, both trajectories are symmetric with regard to the transmitter-receiver axis, so that the shifting in acoustic pulse trajectories does not have an influence on the UA wind speed measurements during the rotation. This conclusion will be observed in the experimental results which will be discussed in chapter 6 .

\subsection{Ultrasonic anemometer in wind turbines}

Wind refers to the movement of air from the high pressure areas to the low pressure areas. Wind power is one of the cleanest and safest sources for generating renewable electricity. A wind turbine is a machine for converting the kinetic energy in wind into the mechanical energy of a rotating shaft. Basically, the rotating mechanical energy is converted immediately by a generator into electrical energy. The generator is connected to the turbine shaft through gears which turn the generator at a different speed than the turbine shaft.

The first law of thermodynamics tells us the energy out of the wind turbine over a certain amount of time has to equal the energy that went into the turbine during the same amount of time. The "energy in" is the kinetic energy from the wind's velocity and air density that flows into the area swept by the turbine blades. It is not possible to convert all of the wind's kinetic energy into mechanical energy. Some energy must remain in the air leaving the turbine. The "energy out" is the energy converted by the turbine blades into mechanical energy (which is then usually turned into electricity), plus whatever energy is left in the air after it passes through the turbine rotors.

Kinetic energy is a function of mass and velocity. So to know the energy in the wind we have to know the wind's velocity as well as the density of the air. Of course, what we are really interested in, as engineers and producers or users of electricity, is the power we can get out of the wind. Power is how fast we are producing or converting a quantity energy which has the units of energy divided by time (Watt).

The equation below shows the variables that determine the power in the wind going into the wind turbine:

$$
P_{w}=\rho \times d^{2} \times U_{\infty}^{3} \times C
$$


where the power $\left(P_{w}\right)$ in the wind going into a specific wind turbine, depends on three variables: density of the air $(\rho)$, diameter of the turbine blades squared $\left(d^{2}\right)$, and velocity of the wind to the third power $\left(U_{\infty}{ }^{3}\right)$. There is also a constant $(C)$ which is actually a combination of two or more constants, depending on the specific variables and system of units. The point is that the variables that determine the wind power blowing into a wind turbine are air density, blade diameter, and wind velocity.

As far as the wind power is dependent on the third power of the wind velocity, knowing the direction of the maximum blowing wind speed in a wind farm is very essential and important for regulating the wind turbine direction towards that incoming wind in order to optimize the wind turbine power and its efficiency.

Therefore, the accuracy in the measurements of wind speed and direction in a wind turbine always is a crucial factor for the wind energy industry. As it is mentioned earlier, ultrasonic anemometer has known as a reliable and well-tested instrument for accurately measurements of the wind speed and direction. Hence, they are used in wind turbine as the nacelle anemometry and spinner anemometry for measuring the wind speed and direction.

Nacelle anemometry consists of cup anemometers, wind vanes and 2D sonic sensors, is commonly used for the wind speed and direction measurements at the rear end of the wind turbine's nacelle. Nacelle anemometry gives input to the control system about wind speed and relative wind direction. This information is used for yaw control, start up and shut down, as well as for power performance verification [14].

The instruments in the nacelle anemometry are influenced by the flow distortion due to the nacelle body and the rotor blade roots. In addition, if the wind is coming at an angle, either horizontally or vertically, the nacelle of the wind turbine introduces vortices and boundary layer effects which may heavily influence the wind sensors so that a correction to the unperturbed wind condition is required. This wind speed correction is dependent on the nacelle's and blade root's geometry as well as the mounting arrangement of the anemometer on the nacelle and must be specified for each type of turbine by means of met mast measurements in the unperturbed air flow. CFD simulations by Zahle et al. [7], have confirmed the measurements sensitivity to flow distortion on the nacelles.

Compared to the nacelle anemometry, it is an advantage to mount the anemometry in front of the rotor, where the flow is undisturbed. Such arrangements were introduced in the 80's on smaller wind turbines which used a rod sticking out from the centre of the hub and carried a cup anemometer and a wind vane (see Fig 3.4 as an example). The rod was 
mounted at the back of the nacelle, and it was carried through the hollow shaft. At the hub it was supported with a bearing. The construction was technically complicated and expensive, and maintenance of the instruments was difficult.

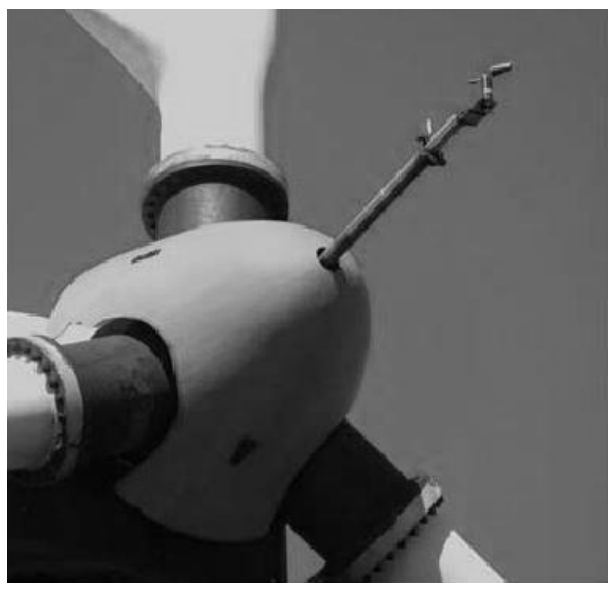

Fig. 3.4: Arrangement of wind sensing instruments on a fixed arm extending from the spinner of a wind turbine.

Recently, a developed alternative to nacelle anemometry is spinner anemometry in which the ultrasonic anemometers are placed over the hub of wind turbine using the flow in front of the spinner for the measurements of wind speed and direction. There are still some distortions in front of the wind turbine, however, the flow distortions on spinner anemometry are rather uncomplicated as compared to the flow distortions experienced by nacelle anemometry [60].

\subsection{Spinner anemometry}

The concept of the spinner anemometry was presented for the first time by Pedersen et al at the EWEC2007 conference [61]. There are two other papers have been written by Pedersen et al. [62, 63], and a report on the CFD simulations [64] in this concept. A spinner anemometry typically consists of three single-axis ultrasonic wind speed sensors, mounted symmetrically on the wind turbine spinner, and an algorithm to convert the wind speeds measured by the three sonic sensors to horizontal wind speed (Uhor), yaw misalignment $(\Gamma)$, and flow inclination angle $(\beta)$, (see the definitions in Fig. 3.5). The yaw misalignment and flow inclination angle are the angles that the wind makes to the rotation axis of the wind turbine respectively in horizontal and vertical plane of the spinner. $U_{h o r}$ is the calibrated horizontal wind speed of the spinner anemometry equals to the horizontal free wind speed $\left(U_{\infty}\right)$ in stopped conditions of the wind turbine. In this thesis, the 
coordinate system in a wind turbine equipped with spinner anemometry has been devoted by XY-plane is the horizontal plane and by XZ-plane is the vertical plane of the wind turbine spinner.

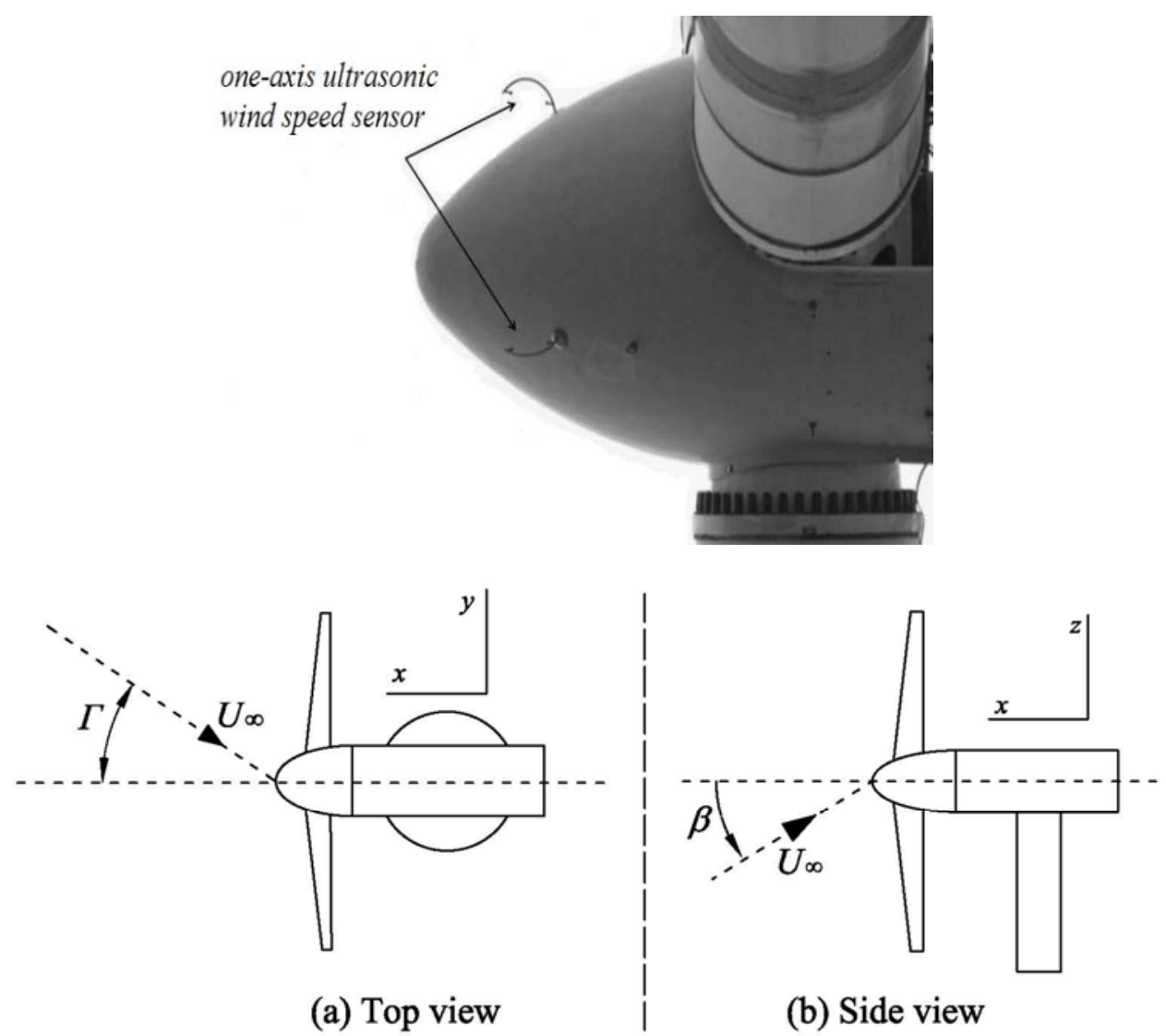

Fig. 3.5: A wind turbine hub equipped with the spinner anemometry; Top view of the wind turbine indicating the yaw misalignment $(\Gamma)$ in the horizontal plane of the spinner (a), and Side view of the wind turbine indicating the flow inclination angle $(\beta)$ in the vertical plane of the spinner (b).

A spinner is normally having a nice aerodynamic shape where the flow behaves in a well described and smooth without separation and thick boundary layers. The three 1D sensors measure the component of the air velocity above the boundary layer of the spinner, in the plane in which the sensors lie and in a direction which is tangential to the surface of the spherical spinner at the point where the sensors are mounted [5]. Spinner anemometry also works well under icing conditions with internal heating of the ultrasonic anemometer and transducers heads [65]. 
Basically, the wind speed and direction can be determined from the two 1D sonic sensors by using knowledge of how the airflow speed is changed in two opposite directions around a spherical object combined with the knowledge of the position of the rotor relative to the horizon. Fig. 3.6 shows a schematic representation of the theoretical flow around a spherical body. According to irrotational flow theory, the tangential airspeed $\left(U_{t}\right)$ of the airflow around a sphere at a certain point $(P)$ can be written as:

$$
U_{t}=U_{\infty} \sin (\varphi)\left(1+\frac{R^{3}}{2 r^{3}}\right)
$$

where $U_{\infty}$ is the velocity of the wind at a sufficient distance from the spinner such that it is not influenced by the spinner, $\varphi$ is the angle formed between a vector from the centre of the sphere to the point of interest $(P)$ and the wind direction vector, $R$ is the radius of the sphere, and $r$ is the distance from the centre of the sphere to the point of interest.

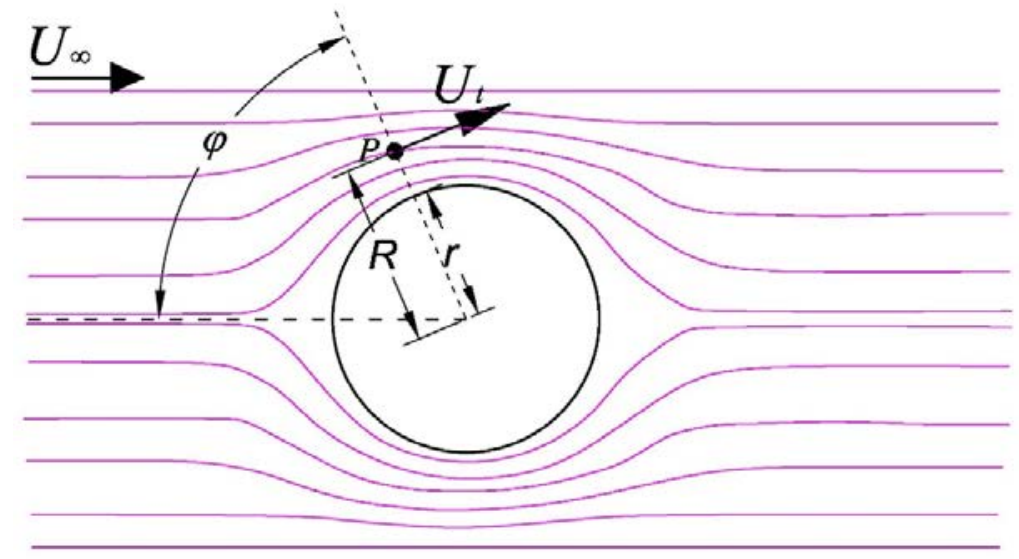

Fig. 3.6: Theoretical streamlines of the flowing around a sphere indicating the wind speed direction and tangential velocity at the point $(P)$.

In the spinner anemometry, two angles are used for calculating the wind direction. These angles are defined as: projection of the wind vector onto the horizontal plane of the wind turbine spinner, $\Theta_{h}$, and projection of the wind vector onto the vertical plane of the wind turbine spinner, $\Theta_{v}$. In the Fig. 3.7 the schematic view of the wind turbine spinner and a single-axis UA is illustrated with the definitions of inflow angle in the horizontal plane of the spinner, $\Theta_{h}$, and in the vertical plane of the spinner, $\Theta_{v}$. 


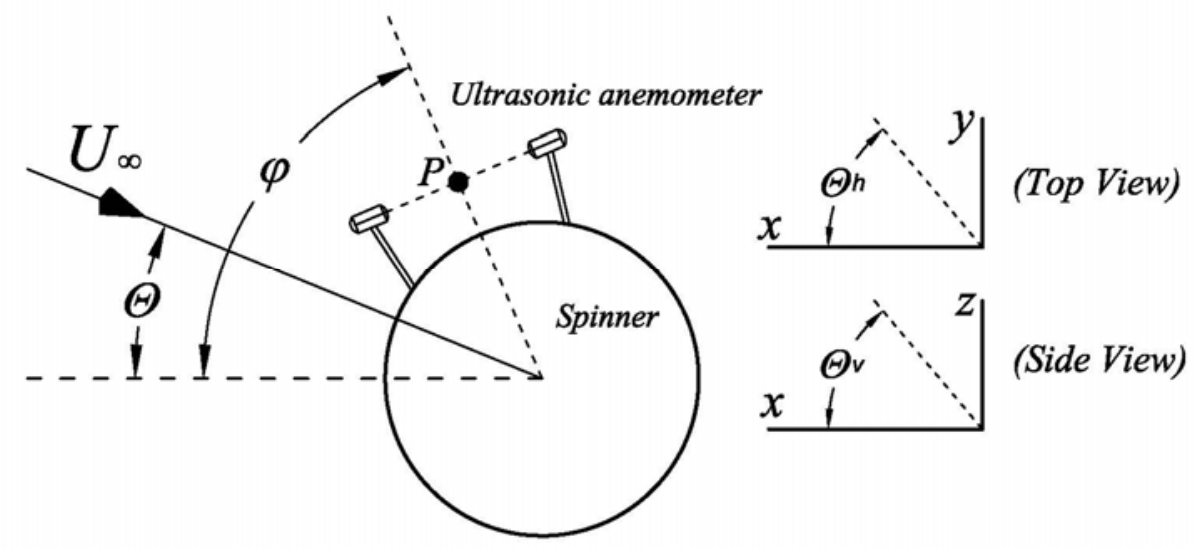

Fig. 3.7: Schematic view of a spinner anemometry indicating the spinner azimuthal position, $\varphi$, of flow stagnation point, and inflow angle, $\Theta$, in the horizontal and vertical plane of the wind turbine spinner with point $(P)$ in the middle of the acoustic measurement path which are identified as $\Theta_{h}$ and $\Theta_{v}$ respectively.

$\Theta_{h}$ is found using the ratio of the wind speeds measured by the two 1D sonic sensors when they lie in the XY-plane. $\Theta_{v}$ is found using the ratio of the wind speeds measured by the two $1 \mathrm{D}$ sonic sensors when they lie in the XZ-plane. Using the $\Theta_{h}$ as an example and the Eq. (3.6) for the tangential wind speed ( $U_{t}$ in Fig. 3.6), the ratio can be calculated as:

$$
\begin{gathered}
F\left(\Theta_{h}\right)=\frac{U_{t 1}\left(\varphi-\Theta_{h}\right)}{U_{t 2}\left(\varphi+\Theta_{h}\right)} \\
F=\frac{U_{\infty} \sin \left(\varphi-\Theta_{h}\right)\left(1+\frac{R^{3}}{2 r^{3}}\right)}{U_{\infty} \sin \left(\varphi+\Theta_{h}\right)\left(1+\frac{R^{3}}{2 r^{3}}\right)} \\
F=\frac{\sin \left(\varphi-\Theta_{h}\right)}{\sin \left(\varphi+\Theta_{h}\right)}
\end{gathered}
$$

The angle $\varphi$ is the angle at which the sensors are placed relative to the rotation axis of the wind turbine spinner and it is constant. As it is seen from the example, the ratio $F$ in the Eq. (3.7) is only a function of $\Theta_{h}$ and not of wind speed. Therefore, by measuring this ratio and using the inverse of the above relationship, the angle $\Theta_{h}$ can be found.

In this example, a closed form solution to the above relationship can be found, see the Eq. (3.8), however, for more complex spinner geometry, it might not be possible to find a closed form solution [5]. 


$$
\Theta_{h}=\arctan \left(\frac{1-F\left(\Theta_{h}\right)}{1+F\left(\Theta_{h}\right)} \tan \varphi\right)
$$

The actual wind speed can be found by putting, for example, the first measured airspeed, $U_{1}$, and the calculated angle, $\Theta_{h}$, into Eq. (3.6) and solving for $U_{\infty}$ :

$$
U_{\infty}=\frac{U_{1}}{\sin \left(\varphi-\Theta_{h}\right)\left(1+\frac{R^{3}}{2 r^{3}}\right)}
$$

$U_{1}$ is the wind speed measured along the sonic path of the first UA which was considered as $U_{t 1}$ in the example above extracted from Eq. (3.7).

In the example presented above, the outputs of the two UAs are recorded in two angular positions over the spinner. The first is when the UA lies in the horizontal plane and the second is when the UA lies in the vertical plane of the spinner. The angular position of the UA can be detected with angular position sensor which continuously measures the angular position of the rotor. Since the UAs are fixed to the rotor, the rotor's angular position and the UAs' angular position are fixed relative to each other. The output of the UAs can therefore be recorded when the angular sensor detects the desired angular position of the rotor. Multiple angular sensors can be used in certain cases to provide redundancy in the case that one or more of the angular sensors fail [5].

For a spinner anemometry three directional wind speeds are measured above the boundary layer in three fixed and axially rotationally symmetric positions. When the wind is coming directly along the shaft axis (X-axis defined in the Fig. 3.5), all wind sensors measure the same wind speed $\left(U_{s 1}=U_{s 2}=U_{s 3}\right)$. Due to the above described principle of the wind speed and direction measurements, when the wind angle of attack on the spinner changes, the three wind speeds at the sensors change. This change is used to determine the wind angle of attack on the spinner including yaw misalignment and flow inclination angle.

In skew incoming air flow, the stagnation point moves away from the nose. A sonic sensor closer to the stagnation point experiences reduced wind speed, while a sensor further away experiences increased wind speed. This will result in a sinusoidal variation in wind speed seen by each sonic sensor during rotation [12].

The wind measurement components of a spinner anemometry are three $1 \mathrm{D}$ sonic sensors having an inclined acoustic path. The sonic sensors are mounted with the sensor paths in plane with the rotor axis and with the sonic paths tilted somewhat backwards to allow wind to come undisturbed into the sensor paths, avoiding sensor head shadow effects; in general form: if the wind is coming towards X-axis of the spinner. In this way, 
the wind component on the sensors due to rotation is cancelled out, and only the wind along the axis direction is measured. The tilted-back configuration of the acoustic path also allows the sensor to be sufficiently narrow so that it can be mounted from the inside of the spinner through a hole in the mounting fitting on the spinner. This is a practical feature that makes mounting and maintenance easy. By converting the three wind measurements of the $1 \mathrm{D}$ sonic wind speed sensors to a representative spinner scalar wind, an angle of attack relative to the rotor axis and an azimuth angle on the spinner, all three wind components relative to a rotating spinner coordinate system are determined. In order to convert the wind components from the spinner coordinate system to a non-rotating stationary coordinate system, fixed with the nacelle, an accurate azimuthal angle, or rotor position, also has to be measured, and used in the conversion.

The spinner anemometry concept comprises some other features. The rotation of the spinner anemometry has the natural consequence that the average values of each sonic sensor, over time, must be the same. This feature can be used to make an "internal" calibration of the sensors against each other. This internal calibration will compensate for inaccuracies of the spinner shape, off-axis mounting, and of inaccuracies of wind sensor mountings. The internal calibration assures that instantaneous wind measurements can be made very accurate. Another consequence of the rotation of the spinner anemometry is that each wind sensor can be used separately to give average wind speed and wind direction values by averaging over time. This makes the system accurate, redundant and very robust [60].

The measurement principle used by the spinner anemometry is actually based on the flow distortion created by the spinner. Because of the size of a modern wind turbine spinner and imperfections of the spinner shape, such as poor rotational symmetry, it is challenging to mount the sensors equally distant from the spinner center and spaced $120^{\circ}$. These challenges in the spinner anemometry mounting could be easily solved in the future by integrating the sensor mounting holes in the spinner mold and by improving the spinner quality control during manufacture of the spinner [13].

\subsection{Calibration of spinner anemometry}

The calibration of a spinner anemometer is somewhat complicated and involves three different calibration phases [12, 13, 14]: 
Phase (1): Wind tunnel calibration; In this test the individual sonic sensors (UAs) are calibrated in an accredited wind tunnel (MEASNET or similar wind-tunnel).

Phase (2): Internal calibration; It has to be performed on every installation of the spinner anemometer. This assumes that the three sonic wind speed sensors must experience the same measured average values over time during rotation.

Phase (3): Turbine-type calibration; As the wind speed and direction measured by the three sonic wind speed sensors will be affected by the geometries of the spinner, nacelle and blade roots, a calibration of the spinner anemometer is needed for every turbine type to convert the wind measured by the sonic wind speed sensor to the free wind affecting the turbine. This calibration can be performed by CFD simulation and by comparing a measurement of the free wind affecting the turbine.

Demurtas et al. [13] concluded that the calibration of the spinner anemometry requires five steps as it is shown in the Table 1. Depending on the intended use of the spinner anemometry, it is not required to execute all the steps. See Table 2 for clarification of which steps are required depending on the intended use of the spinner anemometry.

Table 1: General overview of the sequence steps necessary to calibrate a spinner anemometry (Demurtas and Pedersen [66]). Steps from 1 to 5 are related to the calibration, and the last raw refers to the nacelle transfer function needed to calculate the free wind speed as described in the IEC standard [14].

\begin{tabular}{|c|c|c|}
\hline \multicolumn{3}{|c|}{ Calibration Steps for spinner anemometry } \\
\hline 1 & Zero wind calibration of individual sonic sensors (basic factory calibration) \\
\hline 2 & Wind tunnel calibration of individual sonic sensors (Traceable calibration in wind tunnel) \\
\hline 3 & Internal calibration of the spinner anemometry \\
\hline 4 & Calibration for inflow angle measurements (Calibration for type of spinner anemometry) \\
\hline 5 & Calibration for wind speed measurements (Calibration for type of spinner anemometry) \\
\hline & Nacelle Transfer Function (NTF) & \\
\hline & (IEC 61400-12-2 standard) & Free wind speed \\
\hline
\end{tabular}


Table 2: Indication of which calibration steps should be performed depending on the intended use of the spinner anemometry [13].

\begin{tabular}{|c|c|c|c|}
\hline Calibration steps 1 to 5 & $\begin{array}{c}\text { Wind speed for power } \\
\text { performance measurement } \\
\text { according to IEC 61400-12-2 }\end{array}$ & $\begin{array}{c}\text { Wind speed for } \\
\text { operation of the } \\
\text { wind turbine }\end{array}$ & $\begin{array}{c}\text { Yaw misalignment for } \\
\text { operating of the wind } \\
\text { turbine }\end{array}$ \\
\hline Zero wind calibration & Required & Required & \\
\hline Wind tunnel calibration & Required & Required $^{*}$ \\
\hline $\begin{array}{c}\text { Internal calibration } \\
\text { measurements }\end{array}$ & Required & Required \\
\hline $\begin{array}{c}\text { Calibration for wind speed } \\
\text { measurements }\end{array}$ & Required & Required & \\
\hline Nacelle Transfer Function (NTF) & Required & & \\
\hline
\end{tabular}

* Only required to average out spinner and sonic sensor installation imperfections over a full rotation. For 10 min. average measurements, this calibration has no effect, while it has an effect on turbulence measurements and nonaveraged measurements.

The zero wind calibration is executed by the manufacturer. If power performance measurements are performed according to IEC 61400-12-2, [14], the sonic sensors must be individually calibrated in an accredited wind tunnel. The internal calibration is an automatic calibration made during normal operation of the wind turbine soon after the installation of the spinner anemometry. The calibration for inflow angle measurements and the calibration for wind speed measurements are described in [12, 13].

Pedersen et al. [12] provided a practical example that included and evaluated different methods for calibration of a spinner anemometry for misalignment measurements, i.e. CFD simulations and experimental work by using a met mast in front of the wind turbine spinner. Due to their paper, the internal calibration must be made prior to calibration for angular measurements to smooth out variations during each rotation, so that the rotor azimuth position during calibration yawing does not influence on the calibration of yaw misalignment.

Overall, the calibration process used for spinner anemometry consists of three major phases including the (1) wind tunnel calibration tests for each ultrasonic sensor, (2) the calibration of each spinner UA installations, and (3) the calibration of each wind turbine type.

It is reminded that the objective of this thesis is to propose a calibration process for the spinning $1 \mathrm{D}$ sonic sensor due to its application in spinner anemometry. This is due to 
the calibration process for wind speed and direction measured by each UA, the $1^{\text {st }}$ phase (steps 2 in Table 1), with performing series of tests in an accredited wind tunnel.

It is noted that in the wind tunnel calibration procedure proposed by the IEC 6140012-2 [14], the anemometer is calibrated when the front incoming flow considers laminar, and do not take into account if the wind direction is changing during the measurements.

However, the calibration process for the spinner anemometry proposed in this thesis consists of two phases: (1) A series of wind tunnel tests can be performed for calibrating the 1D sonic sensor used in the spinner anemometry at different stationary yaw or pitch angles of the signal path with wind direction, and (2) A post processing procedure uses the interpolation of the obtained correction factors from the static tests for correcting the wind speed at each angular position of the signal path with wind direction including high yaw or pitch angles. 


\section{Chapter 4}

\section{K-style probe ultrasonic anemometer}

A single-axis ultrasonic anemometer, SATI-1K, serial number: 060803, from Applied Technologies Inc., has been used for the experimental tests in this thesis. It is a microcomputer-based wind sensor capable of measuring wind velocity with reliable accuracy. This chapter provides a complete description about this sonic anemometer including the specifications, fundamental definition of sonic array and operation.

The instrument is designed to measure wind velocity by transmitting and receiving sonic signals along the fixed orthogonal direction. The sonic anemometer offers several major advantages over cup anemometers. Since there are no moving parts to come into dynamic equilibrium with the airflow, the sonic anemometer responds rapidly to wind velocity fluctuations. It responds linearly to wind velocity and is free from contamination from other velocity components as well as pressure, temperature, and relative humidity. The calibration of the sensor is established by its design parameters and, therefore, can be used as an absolute instrument.

This new SATI-1K anemometer incorporates many of the features from earlier models, using state-of-the-art microprocessor and surface mount technologies. The Software that computes the wind velocities has been enhanced and optimized to provide a rapid response sonic anemometer. The electronics that operate the sonic anemometer 
are located in the probe array. The anemometer is DC powered, however other power options are available by external adaptor. The sonic anemometer is connected to the computer running a terminal emulation program, or can be connected directly to a dumb terminal. This is a stable, low powered anemometer, capable of running unattached for extended period of time.

The system detects wind velocity components along mutually orthogonal acoustic path, computes the wind velocity and provides an output directly in engineering units. The system also provides calculations necessary to compute the sonic temperature, corrected for velocity contamination. It provides these data with excellent frequency response and makes it ideal for many aspects of basic atmospheric research.

The probe array's sonic transducers are separated by $15 \mathrm{~cm}$ (acoustic measurement path length). Sonic pulses are generated at the transmitter and receiver by opposing transducers. Mathematics derived for these sonic pulses provide a wind velocity measurement along the acoustic path and calculates a sonic temperature, which is generated from the speed of sound measurements. Temperature measurements are corrected for cross-wind contamination. Fig. 4.1 illustrates the K-style single-axis ultrasonic anemometer from Applied Technologies, Inc. used in the experiments.

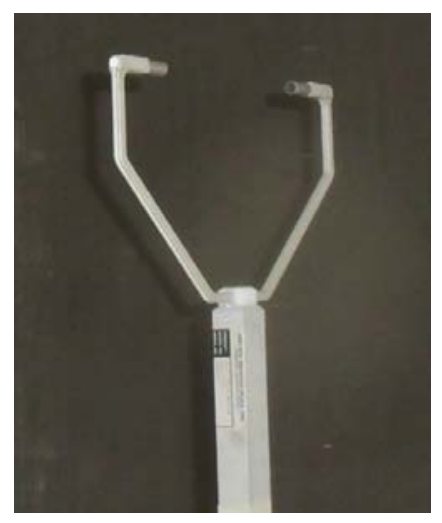

Fig. 4.1: K-style probe single-axis ultrasonic anemometer.

This K-style ultrasonic anemometer has the following specifications:

- Wind speed measurement range: $\pm 30 \mathrm{~m} / \mathrm{s}$

- Accuracy of wind speed: $\pm 0.03 \mathrm{~m} / \mathrm{s}$

- Resolution of wind speed: $0.01 \mathrm{~m} / \mathrm{s}$, wind direction: 0.1 degrees, and temperature: $0.01^{\circ} \mathrm{C}$

- Operating temperature range: $-40^{\circ} \mathrm{C}$ to $+60^{\circ} \mathrm{C}$

- Power requirements: +12 VDC, (+9 to +18 VDC @ 100 mA) 
The anemometer uses a microprocessor-based digital electronic measurement system to control the sample rate, and compute the wind speeds and temperature. The standard data output of the sonic anemometer is $10 \mathrm{~Hz}$, where each output represents the average of 10 discrete measurements. The sample rate and the output rate are user programmable.

Calibration of the sonic anemometer requires the installation of the "Zero-Air Chamber” over the axis being calibrated. A measurement of the ambient air temperature to accuracy of $\pm 1^{\circ} \mathrm{C}$ is necessary for calibration. A calibration command is issued from the computer, and the microprocessor automatically calibrates the sonic anemometer, compensating for any electronic drift. Enter the temperature and relative humidity at the computer terminal when prompted. It is noted that the sonic anemometer is calibrated at the factory before shipping; however, if it may be necessary to perform a Zero-Air Chamber calibration on site.

The software provides the capability of automatic restart in the event of power failure. It detects and displays an error in the data due to blockage of the sonic pulse, and includes some self-diagnostics and reset capability in the event of a microprocessor failure due to outside interference.

The sonic anemometer is designed to run unattended, with very little maintenance required. Since there are no moving parts to contend with, maintaining the sonic anemometer is very simple.

\subsection{Sonic array and operation}

The ultrasonic transducers used in the sonic anemometer are comprised of piezoelectric crystals enclosed in brass housings. The transducers are 3/8 inches in diameter, 7/8 inches long, and are attached to the probe array arms. The transducers are acoustically isolated from the brass housing, and then sealed to prevent exposure to the outside environment. This type of assembly provides transducers that are equipped for rugged outdoor use, and can operate in most environments.

The probe array is a self-contained system, housing the transducers, and operating electronics. An input/output connector enables connection to a data collection computer. The sonic probe array is machined to the specific dimensions required. The tolerance in any dimension is very tight, preserving the orthogonality to 0.1 degree in any direction. The array is rugged sensor assembly capable of tower mounting and normal handling. A 
flat surface on top of the array is provided as a levelling surface during installation. This surface is aligned to the probe array axes to less than 0.1 degrees.

All electronics necessary to the operation of the sonic anemometer are housed in the probe array. Surface mount technology has made it possible to put the electronics on a circuit card, small enough to be located in the mounting arm of the probe array. All connections to the transducers are made inside the probe array, and therefore are completely weather proof. The electronics require DC power which is provided to the array through the cable connector.

The sonic anemometer output is formatted and scaled such that it can be read directly on a terminal. The output of this sonic anemometer can also be used by most any data recorder or computer that has a serial port. The conditions for the serial port are: Full Duplex, 9600 bauds, 7 bit ASCII, even parity and 1 stop bit. The normal output is in meter per second for velocity and degrees centigrade for temperature, but these may be changed by commands issued from the terminal or computer.

The operation of the sonic anemometer is essentially automatic, such that no specific setup procedure is necessary for normal operation. There are, however, commands that may be entered by the operator that will set certain operating parameters. (Refer to the software action of the manual for the commands). The built-in microprocessor power-up sequence sets the system in operation when the power is applied.

\subsection{Hardware installation}

For accurate measurements, the mount should be firm with no slop or backlash. The structure to which the mounting bar is attached should be on the prevailing downwind side of the probe to minimize shadowing of the wind. For best result, the probe should also be mounted to a boom laterally from the structure at least 10 diameters or more.

The installation should be followed by these instructions: secure the probe mounting bar on the structure, feed the output cable through the mounting bar, and connect the end to the probe array. Slide the connector end of the probe array into the mounting bars, lining up the holes in the probe array with the thumbscrews in the mounting bars. Secure the array using the thumbscrews. 


\subsection{Software}

The sonic anemometer software is accessed by using a terminal emulation program such as HyperTerminal from Windows, or any other terminal emulation program. Set the terminal to use the following setting:

- $\quad$ Baud Rate - 9600 bps or 19200 bps

- Parity - EVEN

- $\quad$ Number of data bits -7

- Number of stop bits - 1

- Flow control - NONE

Once the terminal program is connected to the serial port to be used, the sonic anemometer data will be visible. Hit the $\{$ ESC $\}$ key at any time from the terminal to access the sonic anemometer main menu. The sonic anemometer will stop operating while in command mode. The main menu is the first menu available. Seven choices on this menu are described briefly as follows:

\section{A- Output Option}

This menu allows the user to change various options for the sonic anemometer such as baud rate, parity, and type of output desired.

\section{B- Sampling Option}

This menu allows the user to change certain sampling parameters of the sonic anemometer operation, that are toggled ' $\mathrm{ON}$ ', or ' $\mathrm{OFF}$ ', and include the medium filter, data quality algorithm, shadow correction and remove RH from temperature. However, the medium filter is used to return a 'medium' value of ten samples, rather than averaging the samples and reporting the averaged value. Data quality is used to remove spikes caused by various external sources, such as ratio frequency and acoustical interferences. Shadowing correction is used to correct the flow distortion caused by the wind blowing directly along the axis of one transducer. When the RH calculation is on, the RH is used in the equation to calculate the temperature, when toggle is off this factor is removed from the calculation of temperature.

\section{C- Trigger Option}

This menu allows the user to make minor changes to the triggering of the sonic anemometer such as external triggering and character triggering. This command is provided for customers who wish to synchronize the sonic anemometer to other instruments. 


\section{D- Calibration}

This menu is used to perform the calibration of the sonic anemometer. Choose 'Calibration"' when required and enter temperature, relative humidity, and axis to be calibrated from this menu.

E- Current Setting

This choice will display all the current settings for the sonic anemometer. This menu is provided for the user to see exactly how the sonic anemometer is currently configured.

\section{F- Reset Factory Setting}

This menu command restores the original factory set defaults. The sonic anemometer will need to be reset in order to use the new setting.

\section{Z- Factory Setting}

This menu choice contains factory settings. These setting should not be changed unless the operator is instructed to do so by Applied Technologies Inc., technical support. Changing any of the values in this menu could result in inaccurate data, or could make the system inoperable. If in doubt about the settings, then choose 'Restore Factory Settings' from the main menu and reset the system. The two of most important items of this configuration are 'sample per output' and 'ticks per sample' which will be configured for the experiments of the thesis in order to obtain the desired sampling frequency. Samples per output, is the number of high-speed samples averaged (or medium size) to create the desired output. Combining this command with the next command allows adjustment of the output. Tick per sample, sets the number of 'ticks' for each of the highspeed samples. 0 means 5 millisecond ticks, 1 means 10 millisecond ticks, 2 means 15 millisecond ticks, and so on. Combining this parameter with the 'samples per output' sets the recording speed of the instrument. 


\subsection{Summary of the features}

It is claimed by the manufacturer that the SATI k-probe single-axis UA which is used in the experiments of this thesis has the following features listed in Table 3.

Table 3: Features of the K-style probe ultrasonic anemometer.

\begin{tabular}{|l|}
\hline Single component wind velocity \\
\hline Fast response temperature \\
\hline Extreme accuracy \\
\hline Microprocessor-based \\
\hline Solid-state digital operation \\
\hline No moving parts \\
\hline Unattended operation \\
\hline Ease of mounting \\
\hline Rugged construction \\
\hline Low power \\
\hline DC powered \\
\hline Ability to do remote commands through the serial port \\
\hline Perform internal calibration to maintain accuracy \\
\hline User programmable sample rates and averaging, from 5min to 200Hz \\
\hline Exchange averaging with median filter \\
\hline User adjustable data output rates \\
\hline Synchronize sonic operation to external trigger \\
\hline Enter RH value for more accurate temperature output \\
\hline Select data quality algorithm (to remove spikes) \\
\hline User adjustments to data quality calculations \\
\hline Turn flow distortion correction on/or/off \\
\hline User adjustment to flow distortion calculations \\
\hline
\end{tabular}




\section{Chapter 5}

\section{Experimental setup and system design}

This dissertation is an experimental study to investigate the velocity measured by an ultrasonic anemometer in a low speed wind-tunnel performing under two different configurations. This has been carried out under series of static and dynamic tests. In the static tests, the ultrasonic transducers were placed in different stationary azimuthal angles of attack with respect to the wind direction, and in the dynamic tests, the transducers were rotating with various rotational speeds inside the test chamber.

The experiments were performed at the calibration wind-tunnel (S4) of IDR/UPM institute in the Universidad Politécnica de Madrid. This is a low-speed open circuit windtunnel with a closed test section, which has a squared cross section with $0.90 \mathrm{~m}$ side and $1.6 \mathrm{~m}$ length as shown in the Fig. 5.1. The wind tunnel is driven with 4 fans, $7.5 \mathrm{~kW}$ each one. The wind speed is controlled electronically by means of a variable frequency drive, and flow velocities up to $25 \mathrm{~m} / \mathrm{s}$ can be attained with flow uniformity in the testing area better than $0.2 \%$ and turbulence intensity less than $0.1 \%$. The main purpose of this windtunnel is the anemometers calibration fulfills the requirements of MEASNET and ISO/IEC 17025 standard. 


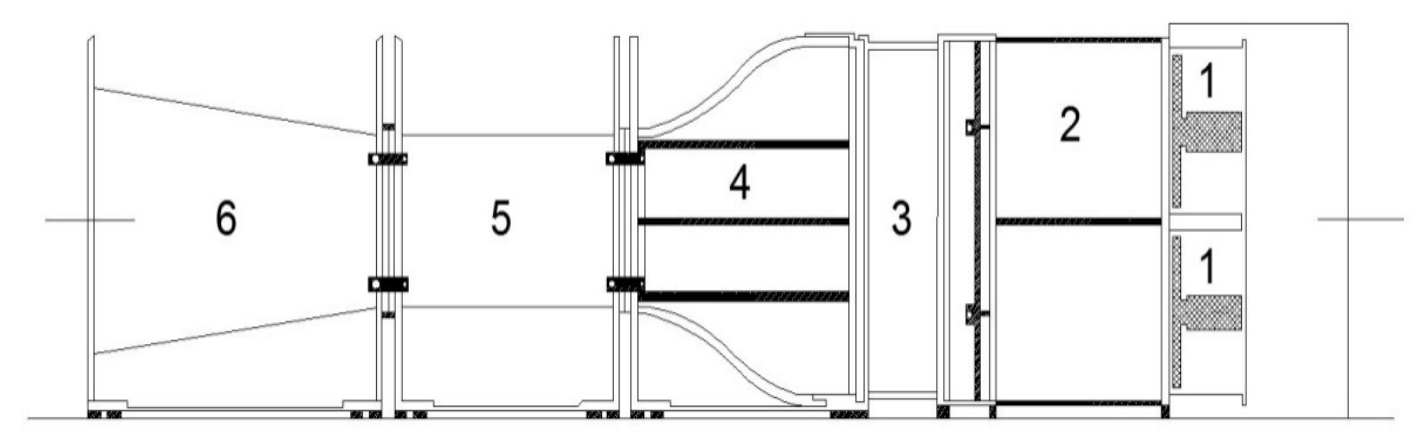

Fig. 5.1: Sketch of the S4 wind tunnel at the IDR/UPM: 1 = fans; 2 = plenum chamber; $3=$ honeycomb and grids; $4=$ contraction; $5=$ test chamber; and $6=$ diffuser.

A specially designed ultrasonic anemometer for investigation has been used for the experiments. It is a modification of the SATI ' $\mathrm{K}$ style-probe' ultrasonic anemometer from Applied Technologies, Inc., (Fig. 4.1). This anemometer has a sonic path length of 0.15 $\mathrm{m}$, a wind speed measurement range of $\pm 30 \mathrm{~m} / \mathrm{s}$ with a resolution of $0.01 \mathrm{~m} / \mathrm{s}$, according to its manual manuscript.

In Table 4, there is a list of used equipment in the wind tunnel which shows the type and the manufacture companies of instruments with their serial numbers. Temperature and humidity sensors are used to determine the air density value. During the tests the wind-tunnel flow velocities are measured with a pitot-tube and a pressure cap. Angular position sensor is used to measure the signal path angle with wind direction. Slip ring capsule is used to transmit electrical current to transducers and download their measurements.

Table 4: List of the used equipment in the experiments.

\begin{tabular}{|c|c|c|c|}
\hline \multirow{2}{*}{ Instrument } & Manufacturer & Type & Serial Number \\
\cline { 1 - 4 } Ultrasonic Anemometer & Applied Technologies Inc & K-SATI & 060803 \\
\hline Angular Position Sensor & Penny \& Giles Controls & NRH280DP & NA \\
\hline Slip Ring Capsule & LTN Servotechnik GmbH & SC020 & NA \\
\cline { 1 - 4 } Pitot Tube & AIRFLOW & 0.48 & N.A \\
\hline Pressure Cap & DRUCK & LPM 9481 & 30064 \\
\hline Digital Multimeter & KEITHLEY & 2000 & 0980771 \\
\hline Barometer Transmitter & \multirow{2}{*}{ VAISALA } & PTU 200 & Z1110002 \\
\cline { 1 - 1 } Temp.-humid. Sensor & HMP45D & Z1110021 \\
\cline { 1 - 1 } Universal Counter & AGILENT & 53131A & MY4003942 \\
\hline
\end{tabular}


The ambient conditions, air temperature, pressure and humidity, have been recorded during the experiments in both static and dynamic tests.

\subsection{Sampling Frequency}

Sampling frequency is an important consideration for any experimental measurements and is measured with the Hertz $(\mathrm{Hz})$, which refers to the number of samples per second. In this experiment, the sampling frequency was taken $50 \mathrm{~Hz}$. As it is mentioned in chapter 4, the 'sample per output' and 'tick per sample' of the used UA are users programmable in order to have the desirable sampling frequency. Accordingly, to have the sample frequency of $50 \mathrm{~Hz}$, it is required to configure 'Factory Setting' option in the sonic anemometer's terminal setting.

From the definition of sampling frequency, the measurement time-step is calculated as $d t=1 / F=1 / 50=0.02 \mathrm{sec}$. According to the UA configuration setting in its manual, the measurement time-step is defined as sample per output multiply by ticks. And tick per sample is defined an integer number related to ticks in millisecond. In Table 5 the relation between tick per sample and tick is illustrated.

Table 5: Configuration of sampling output in UA's serial port.

\begin{tabular}{|c|c|c|c|}
\hline Tick per sample & Ticks & Tick per sample & Ticks \\
\hline 0 & 5 milliseconds & 2 & 15 milliseconds \\
\hline 1 & 10 milliseconds & 3 & 20 milliseconds \\
\hline
\end{tabular}

Therefore, the 'sample per output' and 'tick per sample' are chosen in a way that the desired time-step ( $d t=0.02 \mathrm{sec}$ ) is obtained. Hence, for the experiments 'sample per output' and 'tick per sample' in the configuration setting of ultrasonic anemometer were set as 1 and 3 respectively. Consequently, the time step of the measurements is obtained by $d t=$ 'sample per output' $\times$ 'ticks' $=1 \times 20 / 1000=0.02 \mathrm{sec}$ and therefore, the sample rate is $F=50 \mathrm{~Hz}$. 


\subsection{Experimental setup}

The anemometer was mounted on a structure in a way that its transducers were located in the center of wind-tunnel test section which allowed it to rotate at constant and controlled angular speed. While the UA was rotating, the azimuthal angular positions of transmitterreceiver axis with wind direction, and the wind speed measurements by the UA were recorded and synchronized at the same time. A schematic view of the experimental setup is shown in Fig. 5.2. It is noted that supplying power to the sonic transducers and downloading their measurements increase the degree of complexity of system design. A DC-motor with a controller is coupled by a transmission belt to the UA for providing the desired angular speeds. A contactless angular position sensor, model NRH280DP, manufactured by Penny \& Giles Controls Ltd was used for measuring the instantaneous angular positions of the UA during its rotational motion. A slip-ring-capsule, model SC020, from LTN Servotechnik GmbH, was exploited to supply the electric current to the UA and download its measurements consisting of a rotary part connected to UA and a stator part connected to PC.

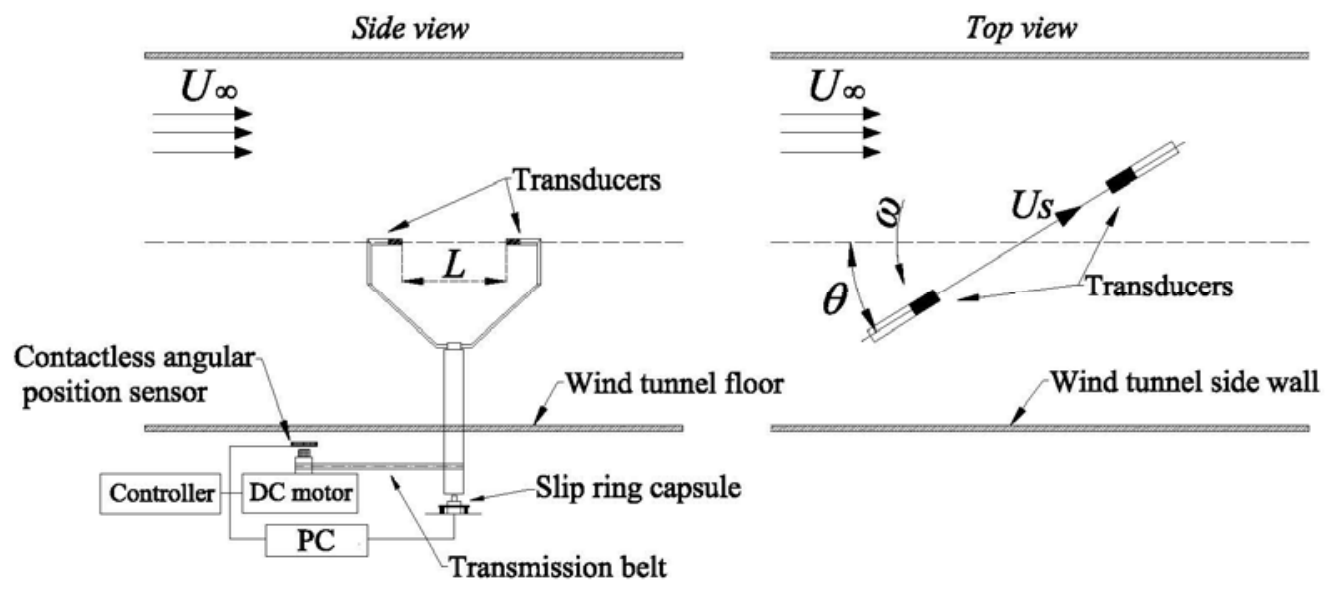

Fig. 5.2: Left: side view of the experimental setup. Right: plan view, definition of ultrasonic path orientation, azimuthal angle, $\theta$, and the direction of the velocity vector measured by the ultrasonic anemometer, $U_{s}$.

Two types of tests have been conducted: static tests and dynamic tests. The static test is similar to a typical calibration of an anemometer, i.e. it is subjected to the wind tunnel flow, varying the wind speed from 4 to $23 \mathrm{~m} / \mathrm{s}$, with a fixed orientation of the acoustic path with respect to the wind-tunnel longitudinal axis, $\theta$ (see the definition in Fig. 5.2). These tests have been repeated for 13 different values of signal path's azimuthal angle $(\theta)$ 
between $0^{\circ}$ and $180^{\circ}$. Measurements from the UA sensor and the angular position sensor were recorded during 42 seconds, at a sample rate of $50 \mathrm{~Hz}$.

In the dynamic tests, a rotational speed is set for the DC motor controller, and various wind speeds are set for the wind-tunnel. At the same time, the wind-tunnel flow velocity measured by pitot-tube, the instantaneous angular position and the wind speed measurements with UA are recorded. This procedure has been repeated for 13 different wind velocities between 4 to $23 \mathrm{~m} / \mathrm{s}$, and for the 12 different rotational speeds of the sonic path as presented in Table 6. The instantaneous angular values were obtained from the angular position sensor. Here, also the outputs of the ultrasonic anemometer and the angular position sensor were recorded during 42 secs, at a sample rate of $50 \mathrm{~Hz}$.

The measuring process started with a 1-minute delay to let the UA sensors and the flow field around reach to a fully stable condition.

Table 6: Tested rotational speeds of the UA sonic path.

\begin{tabular}{|c|c|c|c|c|c|c|c|c|c|c|c|c|}
\hline $\begin{array}{c}\text { Test No. } \\
\text { Tetational speed [rad/s] }\end{array}$ & 1 & 2 & 3 & 4 & 5 & 6 & 7 & 8 & 9 & 10 & 11 & 12 \\
\hline Ro & 1.6 & 3.1 & 3.5 & 4.6 & 5.7 & 6.7 & 8.5 & 9.7 & 11.0 & 13.5 & 23.0 \\
\hline
\end{tabular}

For both static and dynamic tests, Labview was used to record the outputs from the ultrasonic anemometer, and from the angular position sensor.

The Labview code was written in a way to configure the terminal setting of the sonic anemometer due to its manual instruction as illustrated in Table 7.

Table 7: Principle configuration of the UA terminal setting.

\begin{tabular}{|l|}
\hline Bound Rate: 9600 bps \\
\hline Parity: Even \\
\hline Number of Data Bits: 7 \\
\hline Number of Stop Bits: 1 \\
\hline Flow Control: None \\
\hline
\end{tabular}

Once the sonic anemometer is connected to the serial port, the Labview program runs for recording the velocity measured by UA and the angular positions by position sensor. There is a kind of triggering was performed to record the UA velocities and the angular positions at the same time base in each test. 
Fig. 5.3 shows photos of the wind-tunnel test section with UA is installed and ready to be tested. It is shown that the vertical axis of the UA is placed in the middle of test section and two pitot-tubes exist for measuring the free stream inflow velocity. Also in the right photo the structure for holding UA and angular position sensor can be seen beneath the test chamber.

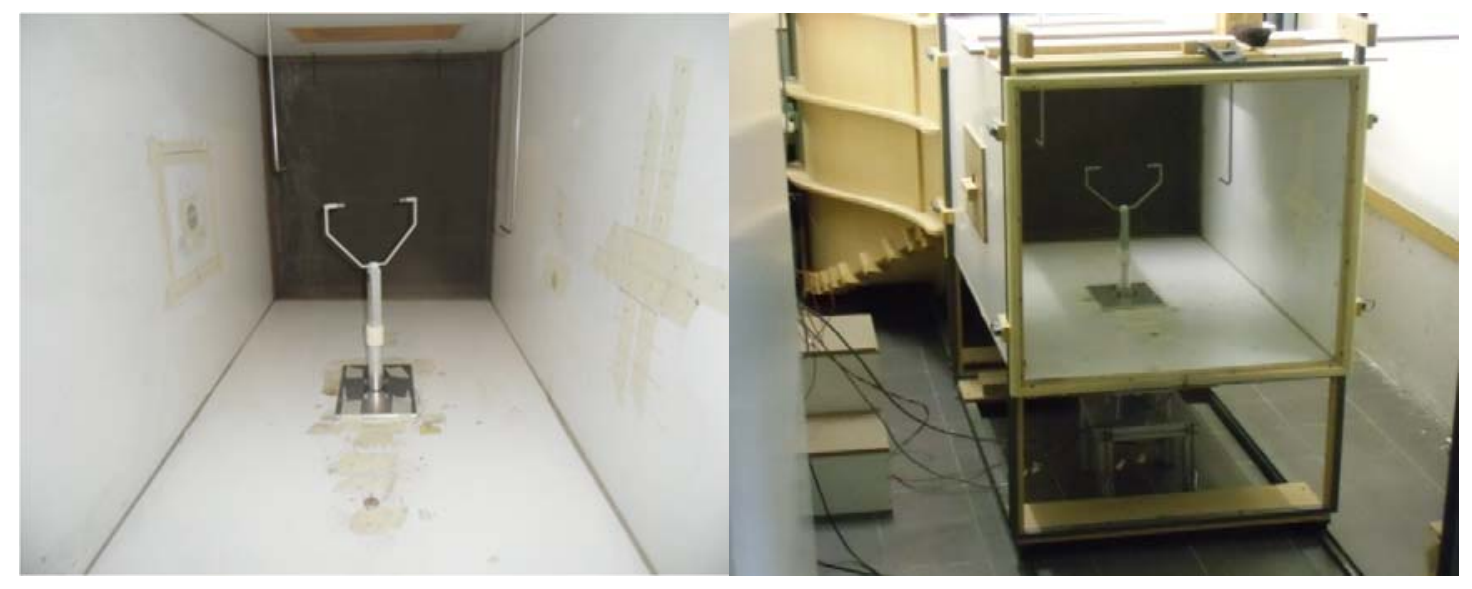

Fig. 5.3: UA is installed in the test section of S4 wind-tunnel ready to be tested.

Apart from the required software for the experiments, it was needed to design and construct a complex mechanical and electrical system. The constructed structure is used for both static and dynamic tests of the experiments taking into account that in the static tests the DC motor is OFF, but in the dynamic tests, the DC motor is ON to provide the required rotational speed. The shaft of the motor is coupled with a transmission belt to the UA. In the following sections, all the instruments, tools, and structural design are described in detail.

\subsection{Structure and System design}

Rotating the anemometer's signal path inside the test section of the wind-tunnel needs a proper system to be designed for holding and rotating the sensors in the middle of test chamber while supplying the electric current and downloading the UA measurements should be taken into account.

The K-style ultrasonic anemometer has three main parts including: The Upper part contains two brackets holding the transducers; the Middle part contains a rectangular aluminium shell having the electronic board inside, and the Down part consists of output connector and cables. 
The design idea is that the Upper part and the Middle part can be rotating during the dynamic tests, while the Down part should be fixed and connected to a PC. Therefore, the upper input and lower output connections of the electronic board should be modified due to the design criterions. On this basis, all the pieces and equipment used in the structure are described in the following subsections.

\subsubsection{Electronic board}

The dimension of electronic board is $270 \mathrm{~mm}$ length and $35 \mathrm{~mm}$ width. As it is seen in Fig. 5.4, the electronic board has two input channels in the left side, and a serial output channel in the right side. The input channels receive the signals from the transducers and the output channel sends the data (velocity measurements) to the computer.

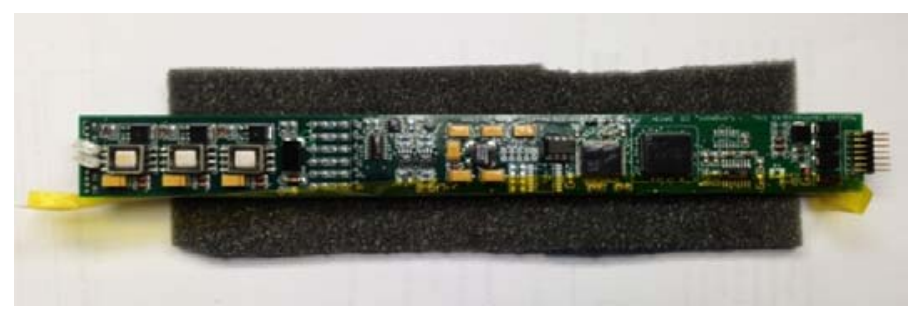

Fig. 5.4: Electronic Board of the tested UA.

\subsubsection{Aluminum tube}

The middle part of the anemometer (see Fig. 4.1) has rectangular section which should be replaced with a circular section such as an aluminium tube. Indeed, the electronic board is supposed to put inside an aluminium tube so that the width of the electronic board determines the inner diameter of the tube. The aluminium tube with inner and outer diameter of $36 \times 40 \mathrm{~mm}$ is selected in which the electronic board can pass through inside the tube. As it is shown in the Fig. 5.5, the tube has a small window hole in an appropriate position where would help us to connect and disconnect the output connection of the electronic board manually. 


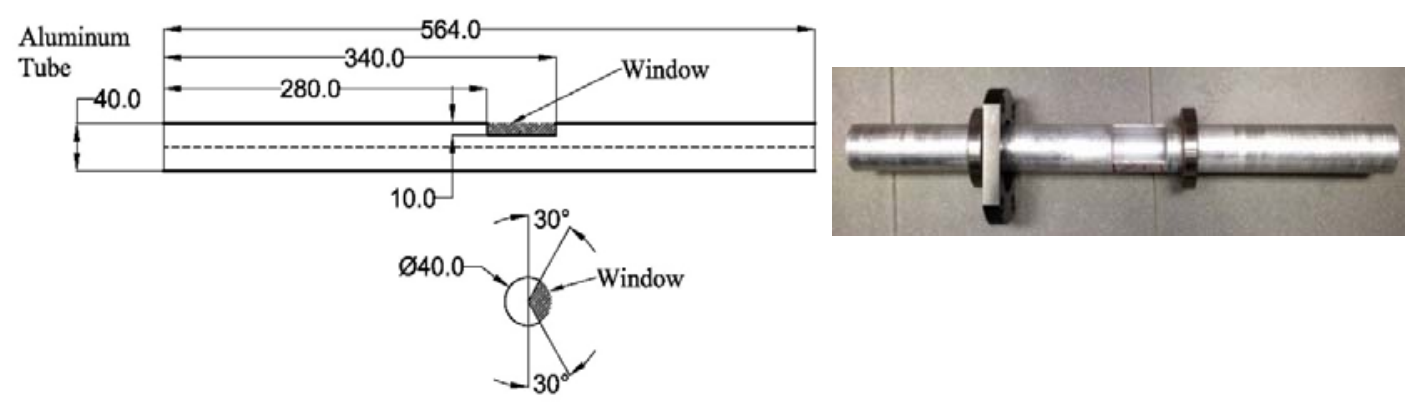

Fig. 5.5: Aluminum Tube (Right) and its scheme draw (Left).

\subsubsection{D upper connection}

The upper part of the UA (see Fig. 4.1) holding the transducers' brackets has a square section. A small piece should be designed to hold the UA upper part with that square section and connect it to the middle part (aluminium tube) with a circular section. This piece is designed with Catia and made by injection 3D printer in the institute's workshop which is shown in Fig. 5.6.
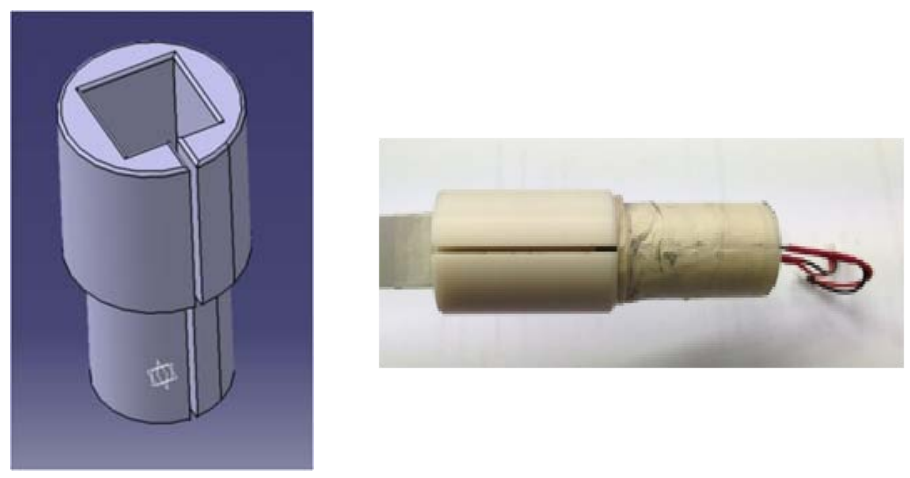

Fig. 5.6: 3D upper connection made by injection printer.

\subsubsection{Input Sockets}

The input channels of the electronic board are modified by adding two sockets. With the help of these sockets, it is possible to connect and disconnect the upper part and middle part of the anemometer whenever it is required. The sockets are illustrated in Fig. 5.7. 


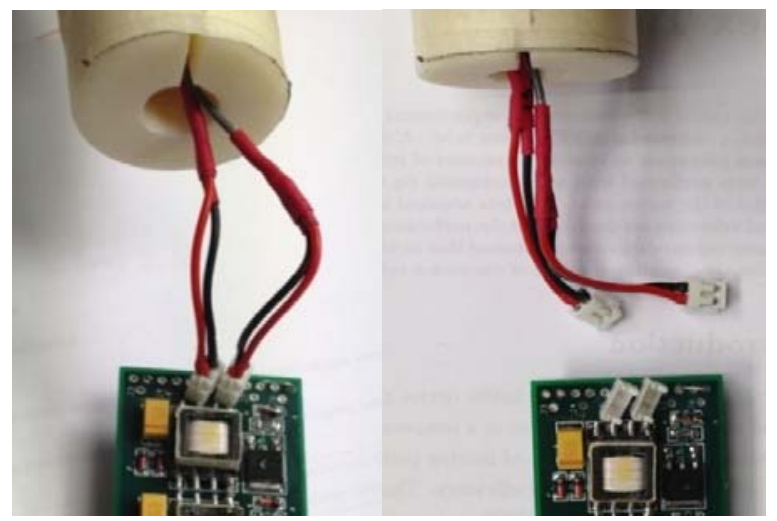

Fig. 5.7: Input Sockets for upper connection.

\subsubsection{Sponge}

For holding the electronic board inside the aluminium tube in a way that can be rotating with the aluminium tube without sliding inside the tube, a thin layer of sponge foam (nonconductive of electricity) was considered to roll around the electronic board to prevent sliding in the interior wall of the aluminium tube. Fig. 5.8 shows the sponge rolled and stuck around the electronic board.

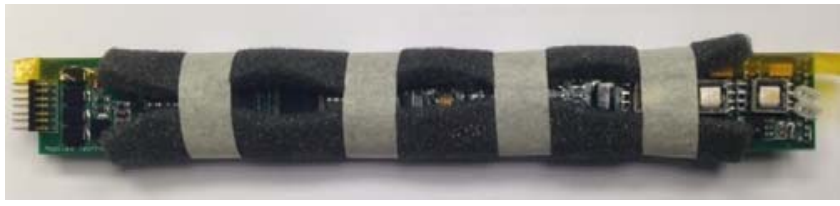

Fig. 5.8: Sponge rolled around the electronic board.

\subsubsection{Slip-Ring-Capsule}

A noticeable tool used in the experiments is 'slip-ring-capsule' which technically helps the data be transferred from a rotary system to a fixed system. The model of this capsule is SC020 manufactured by 'LTN Servotechnik GmbH' company from Germany. This capsule has two parts, rotor and stator, by which the rotary part is connected to the output channel of the electronic board rotating with the electronic board and aluminium tube, and the stator part is fixed and connected to a computer via cable.

The maximum working speed of rotation for this capsule is $250 \mathrm{rpm}$ and it works in the temperature range of $-20^{\circ} \mathrm{C} \ldots+80^{\circ} \mathrm{C}\left(-5^{\circ} \mathrm{F} \ldots+175^{\circ} \mathrm{F}\right)$. Fig. 5.9 illustrates the slipring-capsule. It is seen that there are three holes $\varnothing 5.5 \mathrm{~mm}$ on the stator part of the capsule in order to fix the capsule into a fixed plate. 

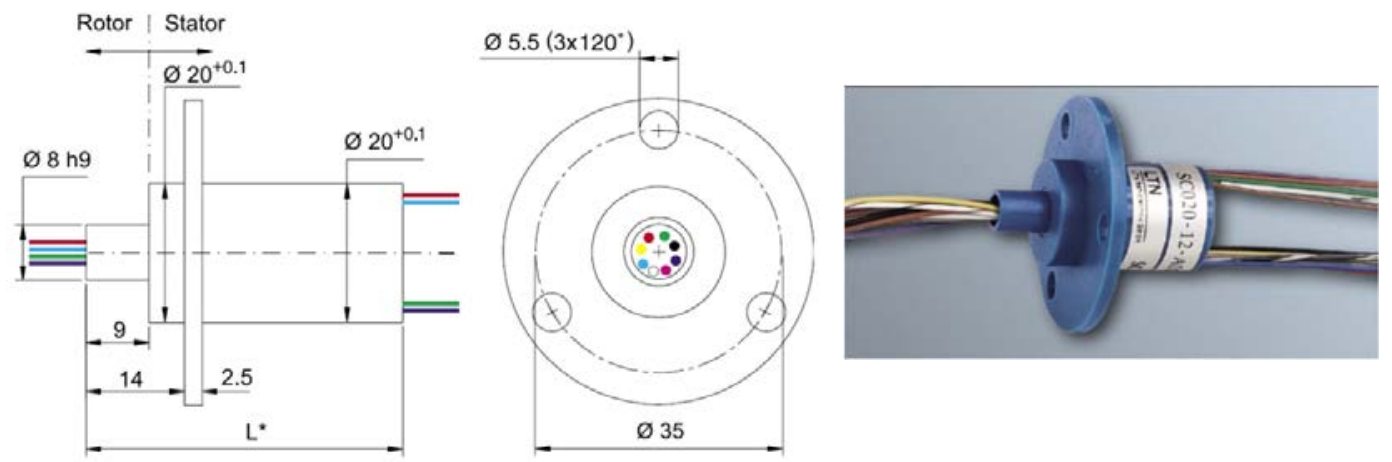

Fig. 5.9: SC020 Slip-Ring-Capsule.

\subsubsection{Angular position sensor}

For both static and dynamic tests of the experiments, the azimuthal angle between the transducers' signal path with wind direction is needed to know. Due to the system design, the aluminium tube is coupled with the DC motor via a transmission belt. The angular position sensor is placed above the DC motor's shaft for measuring the angular positions during the rotation. Fig. 5.10 illustrates the angular position sensor, NRH280DP, manufactured by Curtiss-Wright Controls Co. in US. Due to its data sheet, the distance between bolt and sensor should be set from 2 to $7 \mathrm{~mm}$ for accurate functionality.
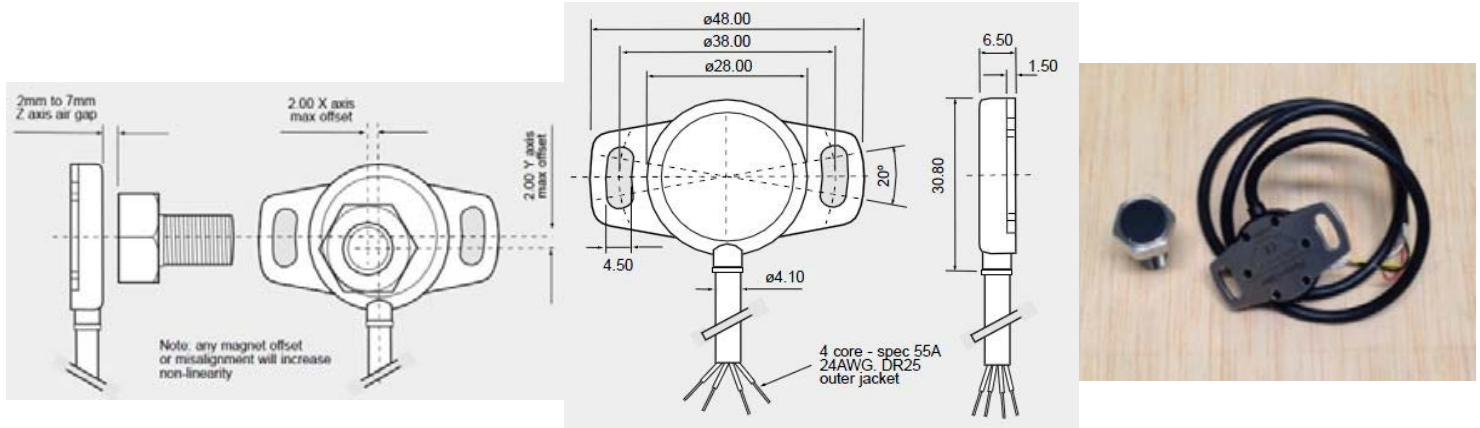

Fig. 5.10: NRH280DP angular position sensor.

The magnetic bolt of the angular position sensor is fixed over the motor's shaft and rotating with the shaft. The sensor is fixed under a rectangular flat plate that holds the sensor in an appropriate distance in accordance with the magnetic bolt (acceptable working range is 2 to $7 \mathrm{~mm}$ ). The sensor reads the angular positions of the magnetic bolt which is equal to the azimuthal angles of the transducers signal path on the other side of the coupled system. Fig. 5.11 shows the motor's shaft and aluminium tube, and the plate for holding the angular position sensor. 


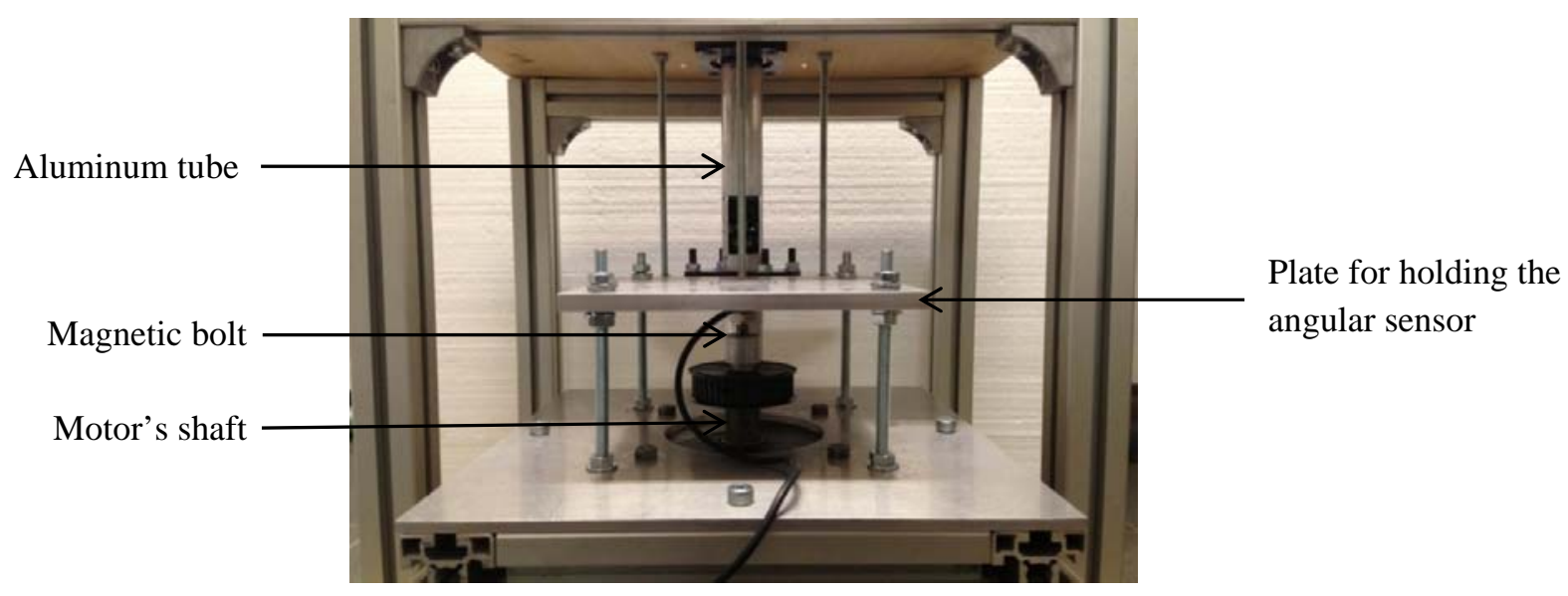

Fig. 5.11: Coupled system between motor's shaft and aluminum tube indicating the position of the angular position sensor and its magnetic bolt.

According to serial number of the angular position sensor used in the experiments: NRH280DP/360/A1/3/B/P5, where '360' stands for the measurement range, 'A1' stands for analogue voltage varies 0.5 - 4.5 (V-Dc), ‘3' stands for clockwise rotation, 'B' means the sensor is bolt type, and ' $\mathrm{P} 5$ ' indicates the cable length is 5 meter.

Fig. 5.12 shows the correlation between output voltages of the position sensor based on the angular positions on the clockwise direction in which the blue line $\left(360^{\circ}\right)$ is for the angular sensor used in this work.

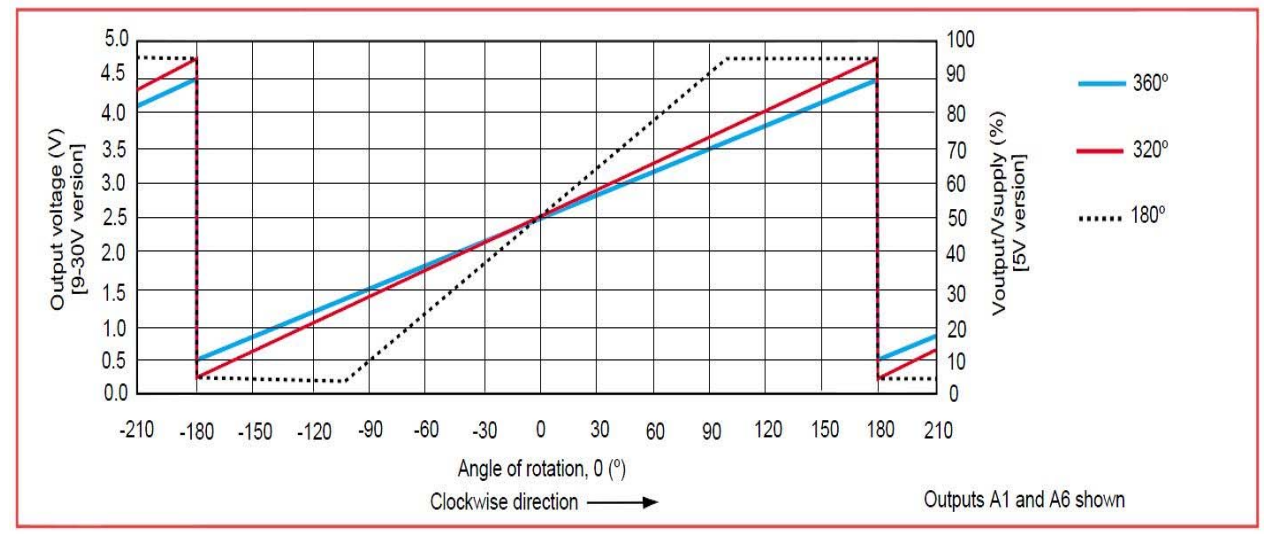

Fig. 5.12: Output voltage of NRH280DP sensor based on the angle of rotation on clockwise direction.

The equation of correlation between the output voltage, $v$, and the angle of rotation, $\theta$, for NRH280DP/360 sensor can be extracted from the graph (Fig. 5.12) as:

$$
v=(1 / 90) \theta+2.5
$$




\subsubsection{SCB-68 National instrument device}

SCB-68 data acquisition board from National Instruments is used for transferring the instantaneous angular position data from the angular sensor to the computer. Fig. 5.13 shows the National Instrument data acquisition device used in the experiments.

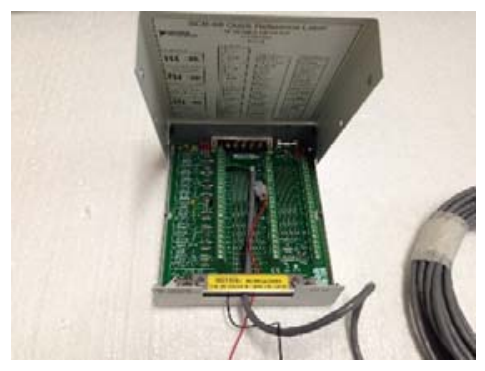

Fig. 5.13: SCB-68 National Instrument data acquisition device.

\subsubsection{Motor and controller}

As it is mentioned before, a DC motor and a controller are used to provide a rotational speed for the UA for each spinning test. The DC motor has 1120 Watt power with the supplier of 100 Volt and 13 Amp. The maximum rotational speed of the motor is 5000 rpm. Different angular speeds were selected with controller for DC motor coupled via a transmission belt to the aluminium tube. Fig. 5.14 shows the photos of DC-motor and controller.
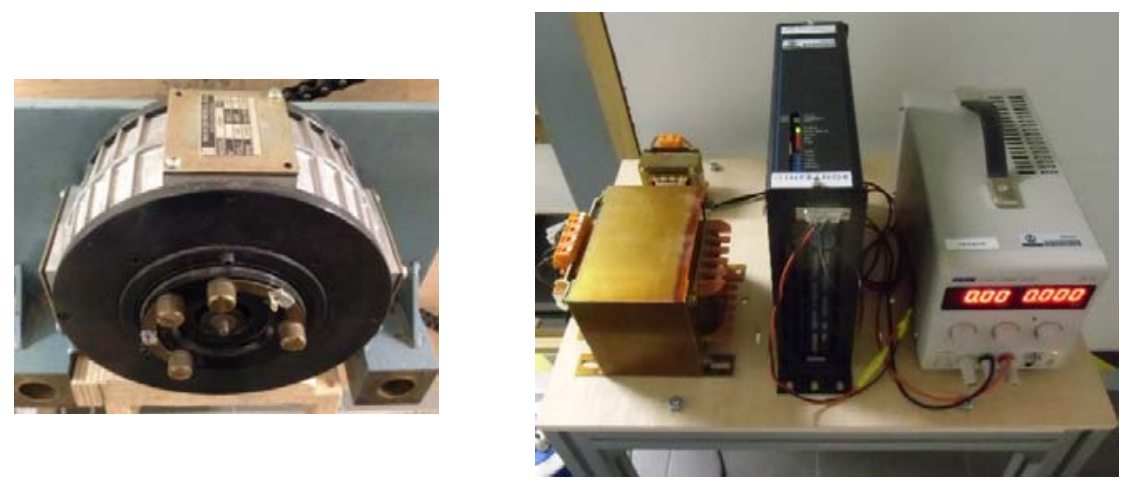

Fig. 5.14: DC motor and Controller. 


\subsubsection{Rectangular plate for holding the aluminium tube}

Fig. 5.15 illustrates an aluminium flat plate (thickness $=10 \mathrm{~mm}$ with dimensions = $200 \times 278 \mathrm{~mm}$ ) which is placed in the middle of wind tunnel test section having four holes ' $30 \times 5 \mathrm{~mm}$ ' for screwing to the test section floor. There is a bigger hole (diameter $\varnothing 42$ $\mathrm{mm}$ ) in the centre of the plate for passing the aluminium tube. A suitable bearing is selected for holding the aluminium tube.

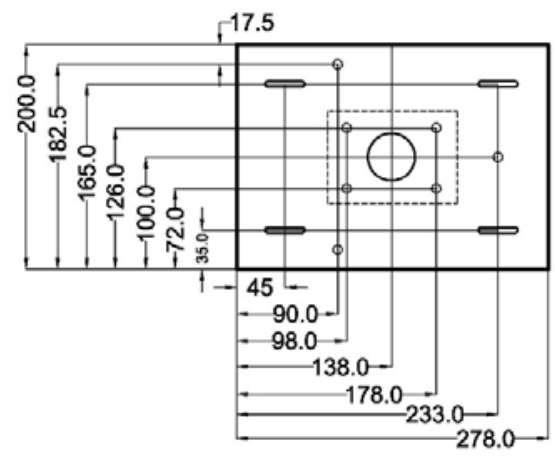

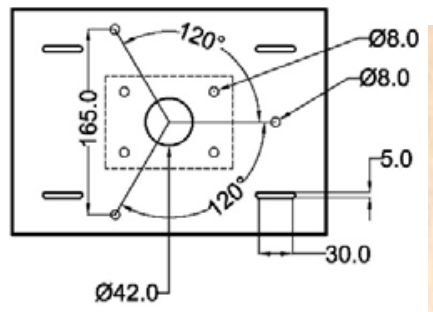

Thickness $=10 \mathrm{~mm}$

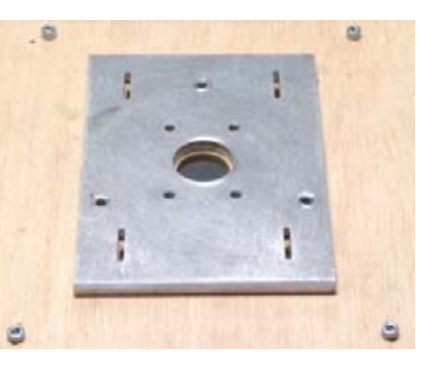

Fig. 5.15: Rectangular flat plate for holding the aluminum tube.

\subsubsection{Triangular plate for holding the aluminium tube}

A triangular flat plate (thickness $10 \mathrm{~mm}$ ) is considered to increase the system rigidity. The base of this plate is $258 \mathrm{~mm}$ and the height is $208 \mathrm{~mm}$. As it is seen in Fig. 5.16, the triangular flat plate has a bigger hole (diameter $\varnothing 42 \mathrm{~mm}$ ) in its centre for passing the aluminium tube using an appropriate bearing.
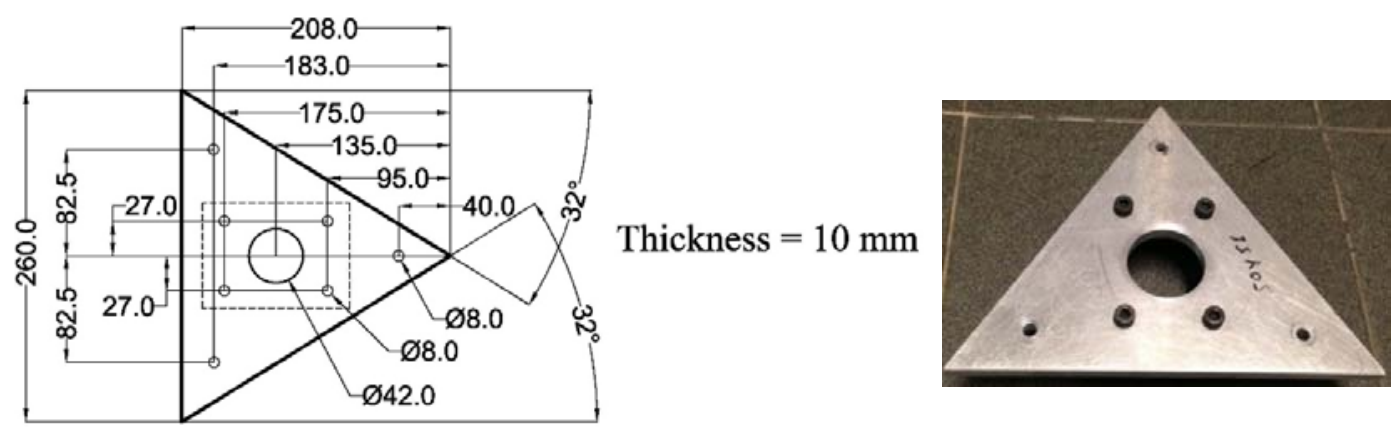

Fig. 5.16: Triangular flat plate for holding the aluminum tube. 


\subsubsection{Beams connection for structural rigidity}

The two above mentioned plates are connected to each other with three beams in order to increases the rigidity of the structure during the experiments. The beams are fixed to each plate with bolts in a way that possible to regulate the distance between the plates. Fig. 5.17 illustrates these three beams with outer diameter of $\varnothing 8 \mathrm{~mm}$.

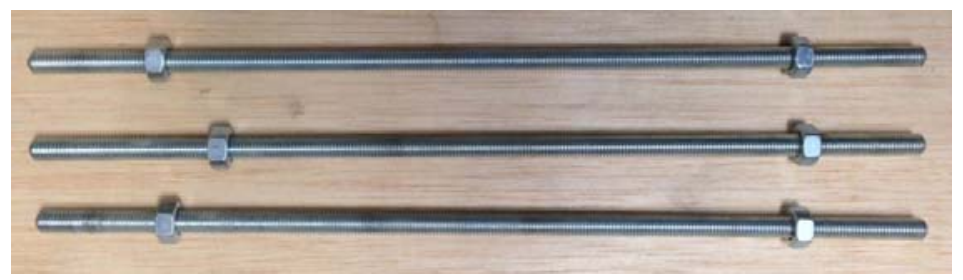

Fig. 5.17: Beams used to connect the rectangualr and triangualr plates.

\subsubsection{Main table and plates}

A table is designed and constructed to hold the DC motor from its upside surface where the shaft comes out. The middle plate of the table holds the DC motor and has a hole (diameter $\varnothing 100 \mathrm{~mm}$ ) in its centre for passing the motor shaft. Additionally, this small table is designed to hold the angular position sensor and slip-ring-capsule. Fig. 5.18 represents this table with two small flat plates connected to the top and left side of the table for holding the angular sensor and slip-ring-capsule respectively.

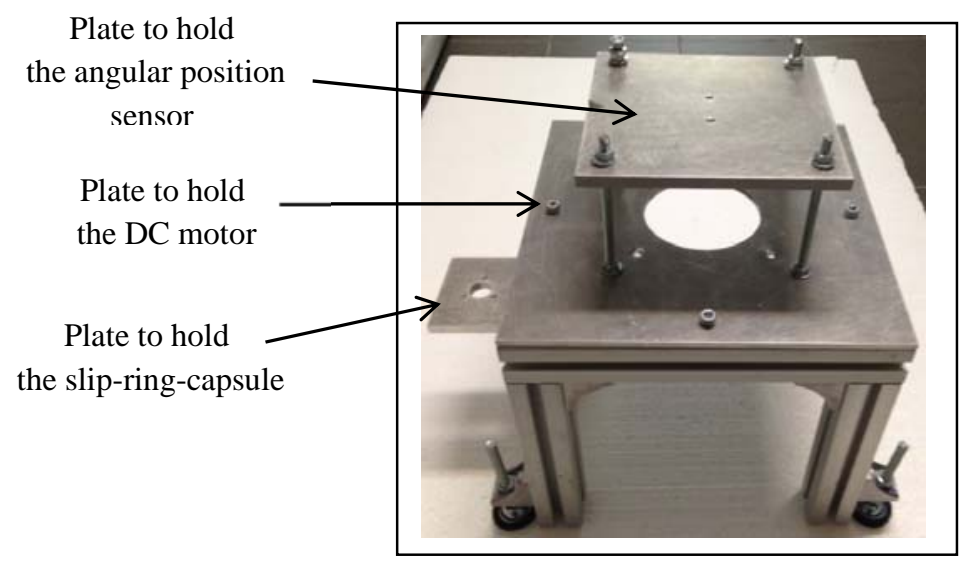

Fig. 5.18: Table to hold the DC-motor, angular position sensor and slip-ring-capsule.

Fig. 5.19 shows the scheme of rectangular flat plate which is installed on the main table for holding the DC-motor. The motor is fixed under the down side of this plate. 


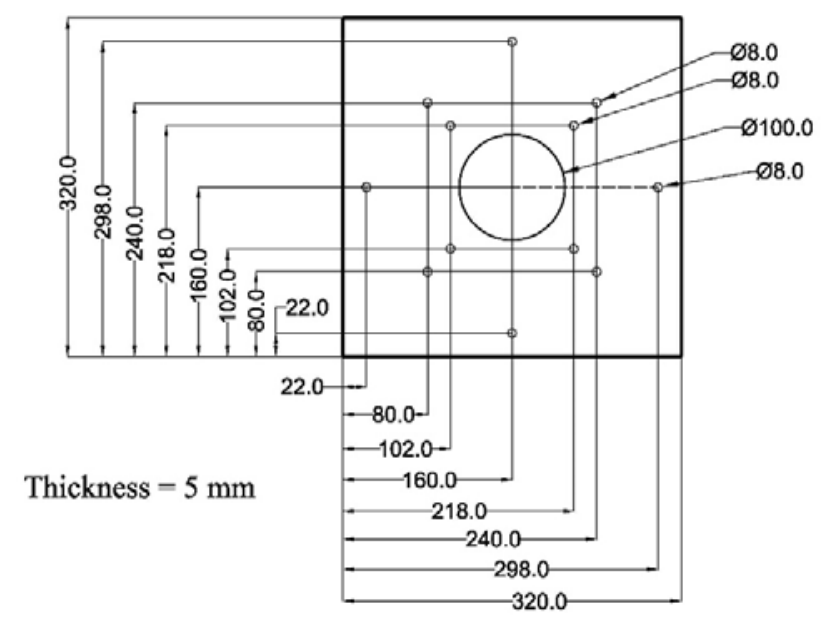

Fig. 5.19: Flat plate for holding the DC-motor.

Due to the functionality of the angular position sensor, a flat plate $(200 \times 200 \mathrm{~mm}$ with thickness of $10 \mathrm{~mm}$ ) is considered to hold the static part of the sensor in appropriate distance from the magnetic bolt. Fig. 5.20 shows this plate which is placed over the middle plate of the main table by four beams (diameter $\varnothing 8 \mathrm{~mm}$ ).

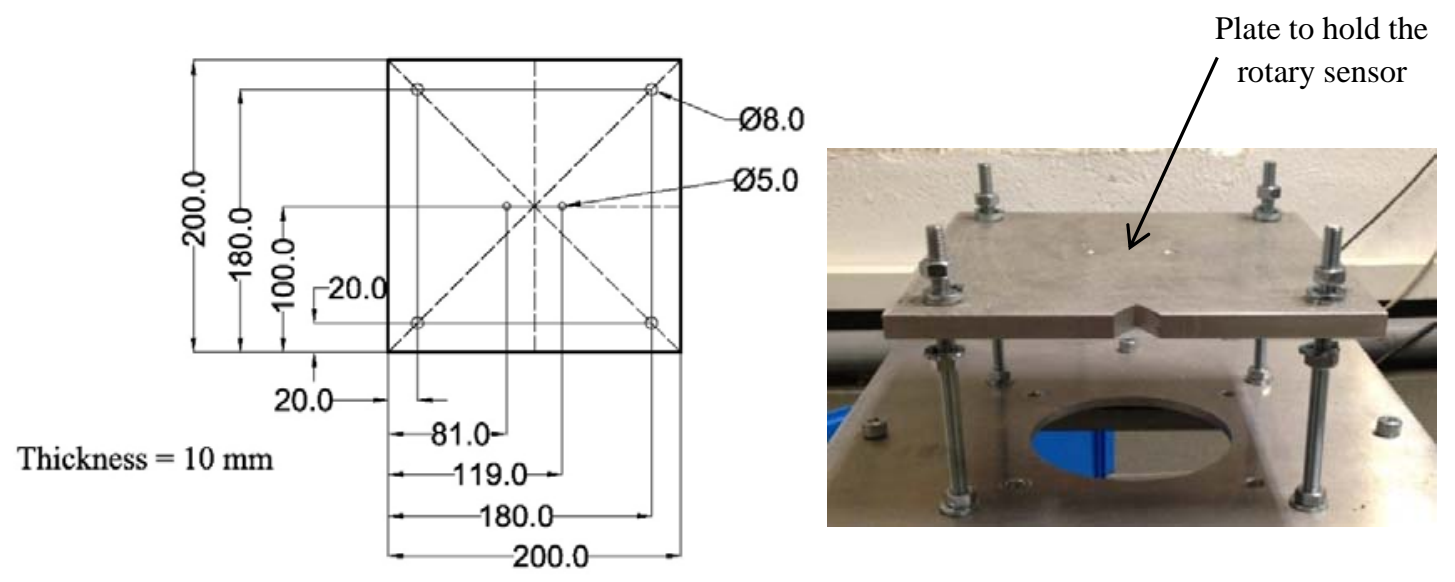

Fig. 5.20: Flat plate for holding the angular position sensor.

Also, it was mentioned that a small plate is designed to hold the slip-ring-capsule. The plate is fixed to the aluminium extrusion of the main table. The plate has a circular hole (diameter Ø22 mm) for passing the rotor part of the slip-ring-capsule. There are three holes (diameter $\varnothing 5.5 \mathrm{~mm}$ ) in symmetric positions to fix the stator part of the slip-ringcapsule to this plate. Fig. 5.21 shows the photo and scheme draw of this plate. 

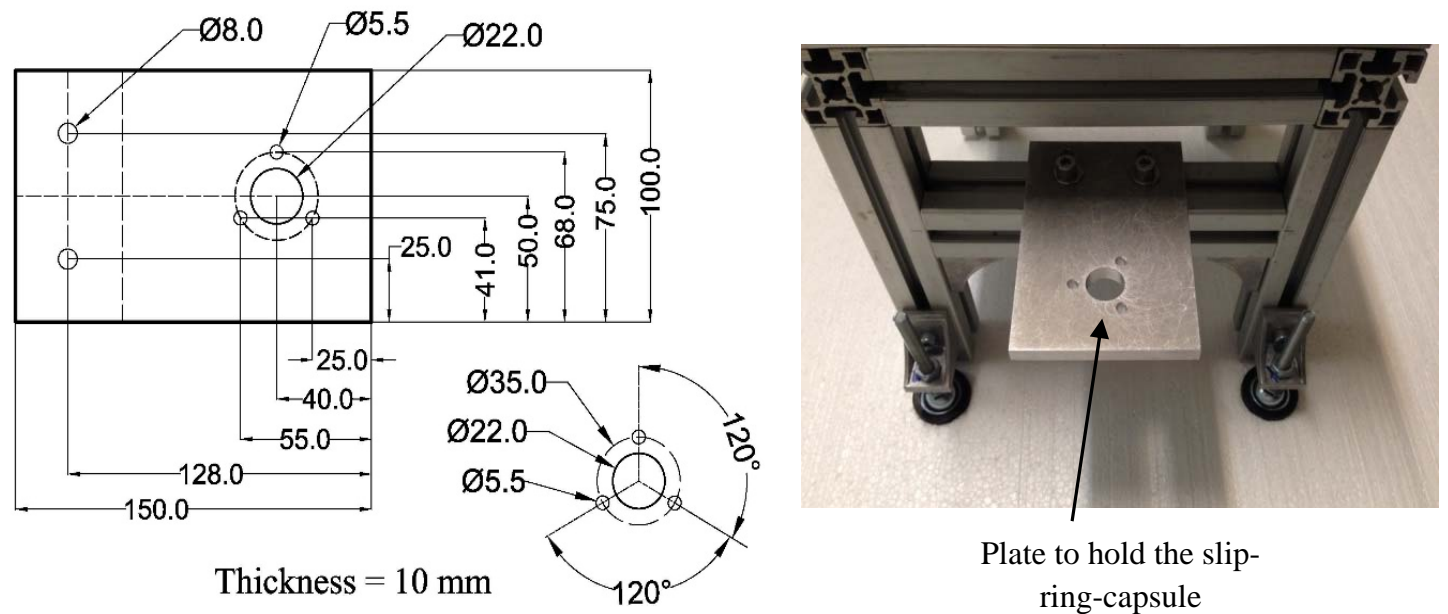

Plate to hold the slipring-capsule

Fig. 5.21: Flat plate for holding the slip-ring-capsule.

\subsection{Mechanical pieces}

\subsubsection{Adjuster pads}

To increase the structural rigidity and reduce the vibrations in the structure during the experiments, the adjuster pads are considered for the table legs connected to the ground. The adjuster pads have $100 \mathrm{~mm}$ adjustment length by which it is possible to adjust the hight of the tables and regulate the position of the UA in the middle of test section. Their stand material is PVC rubber which prevents sliding the legs on the floor. The adjuster pads are bought from MISUMI company (order code: FJGN10-100R) with the coarse, $\mathrm{M}=10 \mathrm{~mm}$, and adjustment length, $\mathrm{A}=100 \mathrm{~mm}$. Their rubber is conductive with specific volume resistivity: 102-104 $\Omega . \mathrm{cm}$. And their screw shaft turns independently of the base and tilts slightly. In Fig. 5.22, the photo and characteristic of these adjuster pads are illustrated.

\begin{tabular}{|c|c|c|c|c|c|c|}
\hline$M$ & D & $\ell_{1}$ & $\ell_{2}$ & $E$ & B & $\mathrm{H}$ \\
\hline 8 & \multirow{2}{*}{45} & 5.5 & 6.5 & \multirow{2}{*}{15} & 13 & 6 \\
\hline 10 & & 7 & 8 & & 17 & 6 \\
\hline 12 & 70 & 8 & 10 & 23 & 19 & 7 \\
\hline 16 & \multirow{2}{*}{80} & 10 & 13 & \multirow{2}{*}{25} & 24 & \multirow{2}{*}{8} \\
\hline 20 & & 13 & 16 & & 30 & \\
\hline
\end{tabular}
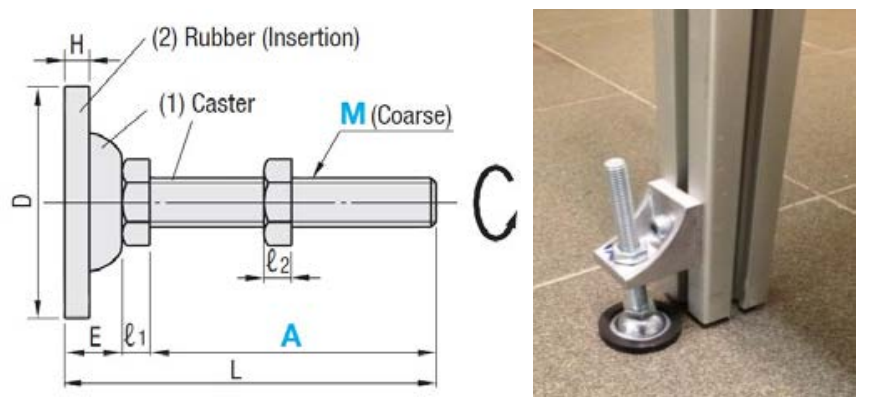

Fig. 5.22: Non-slip adjuster pads for the main table. 


\subsubsection{Bearings}

Two bearings are selected for the aluminium tube; one is considered for the rectangular flat plate placing on the floor of wind tunnel test section, and the other one is considered for the triangular flat plate. The compact type bearing with rubber sealed is selected and ordered from the MISUMI Company (order code: BGCNB 6008 DD). The material of the bearing is steel, and the surface treatment is black oxide. Fig. 5.23 illustrates the schematic view of the selected bearings. In this case, $d$ is the outer diameter of the aluminium tube $(40 \mathrm{~mm})$, and the other parameters are defined as: $A=80 \mathrm{~mm}, \mathrm{~F}=54 \mathrm{~mm}$, $\mathrm{W}=82 \mathrm{~mm}$, and $\mathrm{M}$ (Coarse, $\left.\mathrm{d}_{1}=14 \mathrm{~mm}, \mathrm{H}_{1}=8.6 \mathrm{~mm}\right)$.

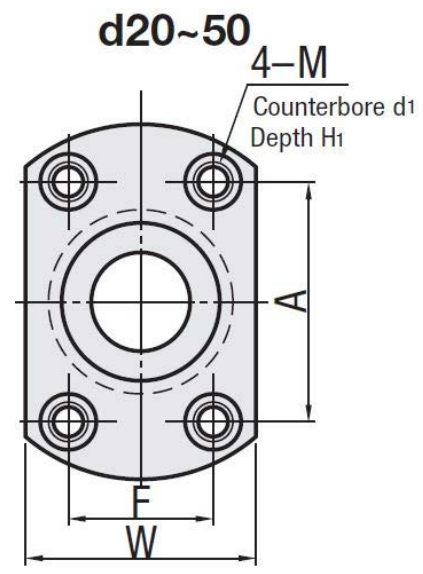

Fig. 5.23: Bearings for holding the aluminum tube.

\subsubsection{Pulleys}

Due to the outer diameter of motor's shaft (24 mm) and the outer diameter of aluminium tube $(40 \mathrm{~mm})$, two pulleys are chosen from MISUMI catalogue from the 5GT type pulleys. The order codes to buy the pulleys were:

- GPM44GT5150 A P24

- GPM44GT5150 A P40

The 'A' shape and ' $P$ ' type is selected, with a set screw holes at around $120^{\circ}$ for tightening them to the shaft and aluminium tube. Fig. 5.24 shows the photo and characteristic of the selected pulleys. 

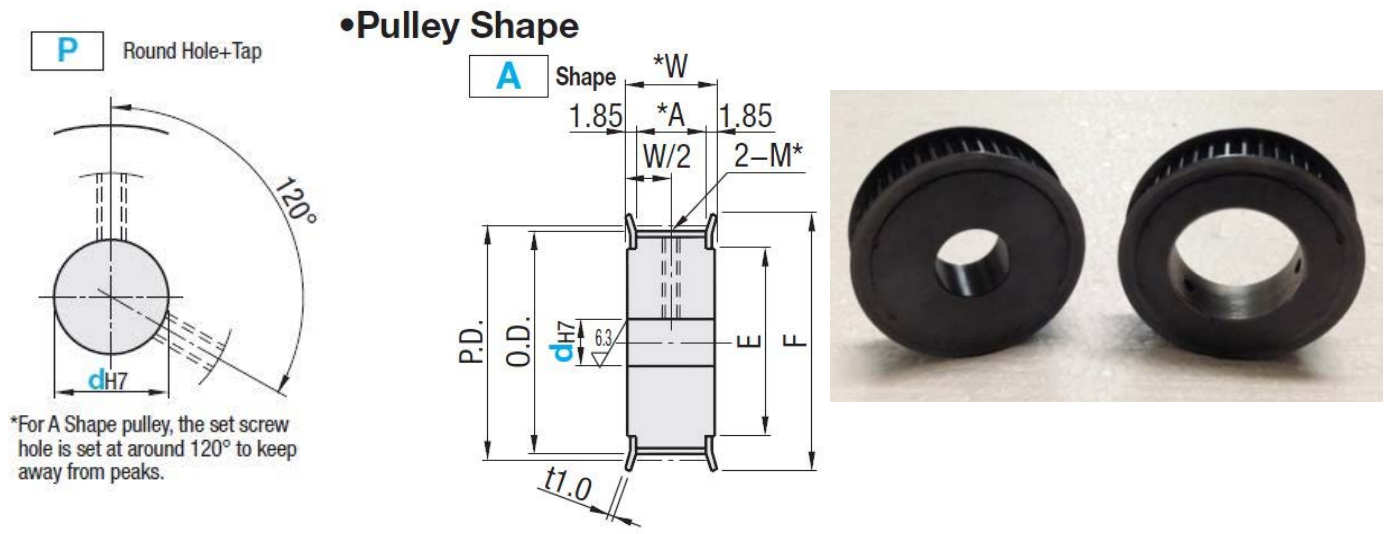

Fig. 5.24: 5GT-type Pulleys.

\subsubsection{Transmission Belt}

To choose the transmission belt, it is necessary to know about the geometric relationships between belt and pulleys. In the Appendix A, the general rules of the selecting a belt regarding to the pulley is presented. Regarding that the belt characteristics are calculated as below:

Due to the characteristics of the pulleys (5GT Type), $\mathrm{P}=0.5 \mathrm{~mm}$ and $\mathrm{ZP}_{\mathrm{P}}=44$, the pulley pitch diameter is obtained as: $d=P \times Z P / \pi=70.03$. And since, the pulley pitch diameter is the same for both pulleys $\left(d_{2}=d_{1}=70.03\right), d_{2-} d_{1}=0$, according to Appendix A, Eq. (A.9), the distance between two shafts is $C=b / 4$. Besides, from the structural design, the temporary shaft distance, $C^{\prime}$, could be estimated as $225 \mathrm{~mm}$.

$L_{P}^{\prime}=2 C^{\prime}+\frac{\pi\left(d_{2}+d_{1}\right)}{2}+\frac{\left(d_{2}-d_{1}\right)^{2}}{4 C^{\prime}}=2 \times 225+\pi \times 70.03=670.0057 \mathrm{~mm}$

From the MISUMI catalogue, the nearest value to $L_{p}{ }^{\prime}$, was found as $L_{p}=670 \mathrm{~mm}$. Hence, the correction factor $(b)$ is given as:

$b=2 L_{p}-\pi\left(d_{2}+d_{1}\right)=2 \times 670-2 \pi \times 70.03=899.988$

Therefore, the inter-shaft distance for the selected belt is calculated as $C=b / 4=224.997$ which is considered as $225 \mathrm{~mm}$ to choose the belt from the catalogue. So, the transmission belt was ordered (code: GBN670EV5GT-150) from MISUMI. The belt operating temperature is $-10 \sim 80{ }^{\circ} \mathrm{C}$, and its specifications are illustrated in Fig. 5.25. 


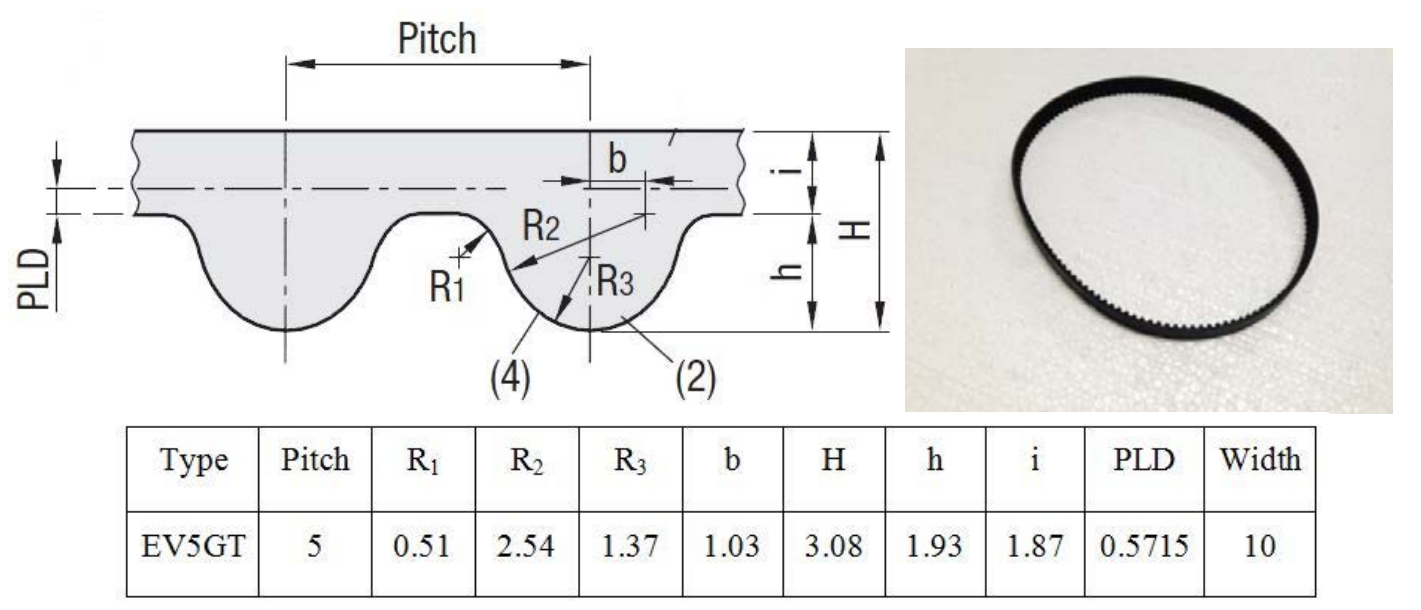

Fig. 5.25: Super high torque timing belt, EV5GT.

\subsection{Summary of structural design}

The structure is designed and constructed in way that it had a negligible vibration during the rotational tests. The anemometer was fixed from two points with two suitable bearings to the flat plates. The distance between these two points was long enough to avoid misalignment in the vertical axis of the anemometer during the rotation. Furthermore, the motor, as the main source of vibration, was installed on a table with four legs, each one connected to the ground floor with an adjuster pad (Fig. 5.22). These adjuster pads were made of PVC rubber which prevents sliding. Also, the table was too heavy to transmit the probable motor vibrations to the adjuster pads. The transmission belt for connecting the anemometer to the motor's shaft, on the other hand, was short and tight with no looseness and extra degree of freedom, avoiding any vibration in the coupled system. It can thus be claimed that there was no remarkable vibration in the entire system due to the rotating parts. Visual investigations also approved it.

Also, it can be said that the acoustic pulses travel with the speed of sound which is by far greater than the UA rotational speeds so that the negligible vibration cannot affect the anemometer measurements during the rotational tests.

The schematic draw of the whole system is illustrated in Fig. 5.26. This figure shows the instruments and mechanical pieces with some dimensions and distances. 


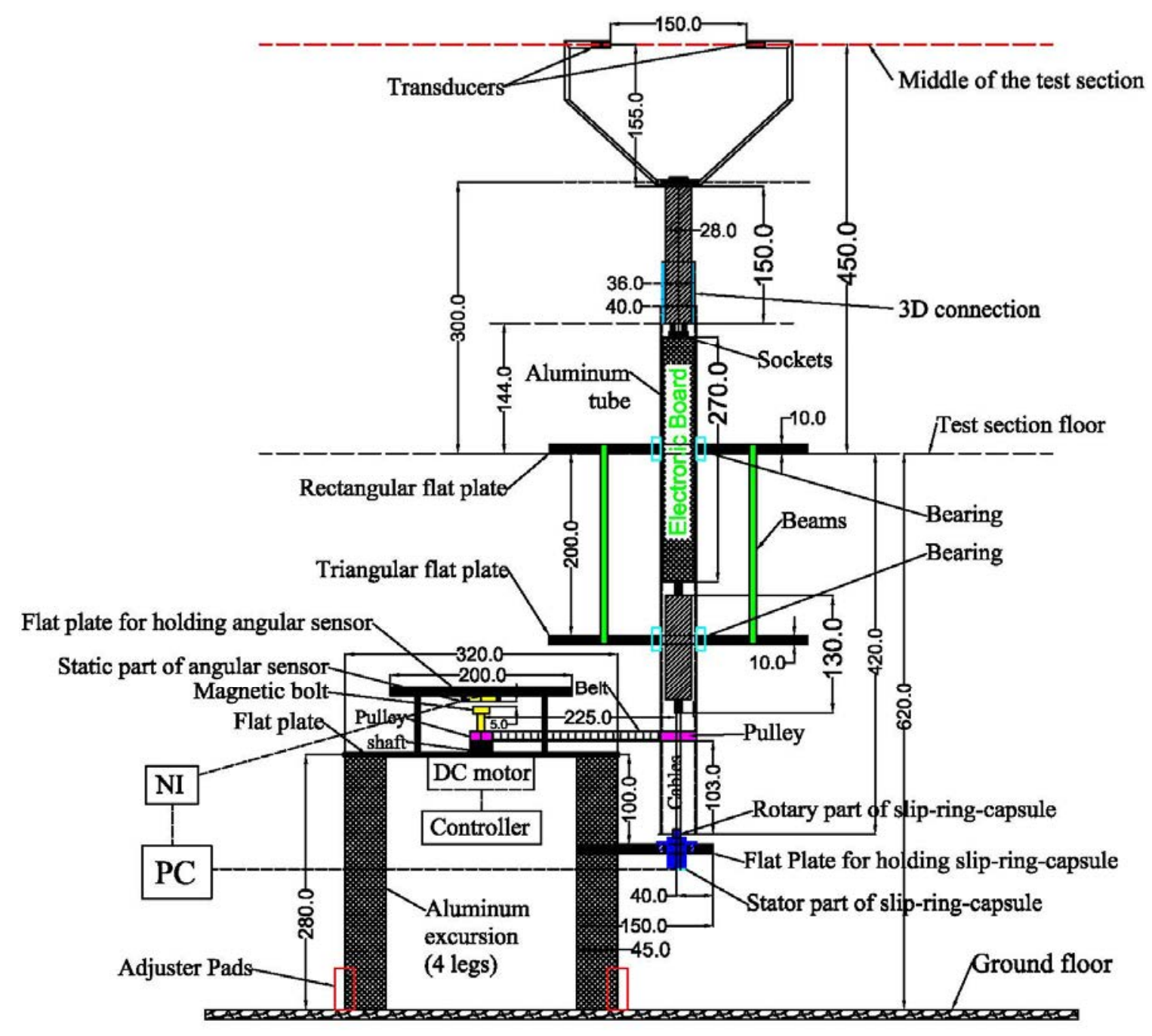

Fig. 5.26: Schematic draw of the whole system with detail dimensions. 


\section{Chapter 6}

\section{Experiments and results}

In this chapter a calibration process for a modified k-style probe single-axis ultrasonic anemometer, is proposed for its wind speed measurements in static and dynamic conditions. The chapter starts with the methodology and the idea of calibration process, continues with the static directional calibration tests, follows by an uncertainty analysis and accuracy of measurements, and then the calibration of measurements in dynamic conditions will be discussed. Due to the obtained results, the 1D sonic sensors used in the spinner anemometry can be calibrated with a similar wind-tunnel calibration process proposed in this chapter.

\subsection{Methodology and calibration process}

The idea of the calibration process for the wind speed measured by a single-axis ultrasonic anemometer in rotational motion consists of two phases. Firstly, the anemometer is calibrated for different stationary inflow angles. Secondly the interpolation of the obtained correction factors is used for the calibration of ultrasonic wind speed measurements in rotational motion. In the static tests, anemometer's signal path was placed in different stationary angles of attack with respect to the wind direction, and 
different wind speeds were set for the wind tunnel in order to calibrate the UA measurements with the reference measurements obtained by pitot-tube.

In the dynamic tests, the ultrasonic anemometer was rotating inside the test chamber of the wind tunnel with various rotational speeds. For each UA angular speed, different wind speeds were set for the wind tunnel. As it was mentioned in chapter 5, a complex system was designed for supplying power and downloading the measurements from the spinning ultrasonic anemometer. The interpolation of obtained correction factors from the static tests is used to calibrate the measurement data in the dynamic tests which validate the idea of calibration process.

\subsection{Static tests}

In the static tests, the signal path was placed in the test section in 13 different stationary angles of attack $\left(0^{\circ}, 5^{\circ}, 10^{\circ}, 15^{\circ}, 30^{\circ}, 60^{\circ}, 90^{\circ}, 120^{\circ}, 150^{\circ}, 165^{\circ}, 170^{\circ}, 175^{\circ}\right.$, and $\left.180^{\circ}\right)$ and 9 different wind speeds $\left(U_{\infty}\right)$ between 4 - $24 \mathrm{~m} / \mathrm{s}$ were selected for each test. The angle of attack is defined as the azimuthal angle $(\theta)$ between transducers' signal path with respect to flow direction on counter clockwise rotation. The outputs of the static tests were the wind velocity measured by UA and the wind speed measured by pitot-tube, for each fixed signal path direction (see the definition of azimuthal angle, $\theta$, in Fig. 5.2).

The correlation between voltage and azimuthal angle, Eq. (5.1), is used to find the position of the signal path inside the test chamber. Each selected azimuthal angle is substituted into ' $\theta$ ' and its voltage ' $v$ ' is calculated from the Eq. (5.1) which uses to set manually the transducers in its appropriate position where the voltmeter also shows the same voltage. This setting is done when the wind-tunnel is off and the influences of external noises on the voltage readings is very negligible.

The results of the static tests are illustrated in Fig. 6.1, where the ultrasonic velocity measurements, $U_{s}$, at different stationary azimuthal angles are plotted based on the free stream wind velocities, $U_{\infty}$. It is shown that the velocity measurements by UA have positive and negative values at different azimuth angles. The positive values are for the angles from $0^{\circ}$ to $90^{\circ}$, and the negative values are for the angles $90^{\circ}$ to $180^{\circ}$, so that the measurements are symmetric around $90^{\circ}$. The UA at the azimuthal angle of $90^{\circ}$ measures almost zero wind speed for all the different wind speeds. Because, at this critical angle the signal path is perpendicular to the inflow direction, so that the acoustic pulse travelling 
time in forward and backward directions are almost equals, $t_{+} \approx t$-, and due to the Eq. (3.2) the anemometer measures almost zero wind speed.

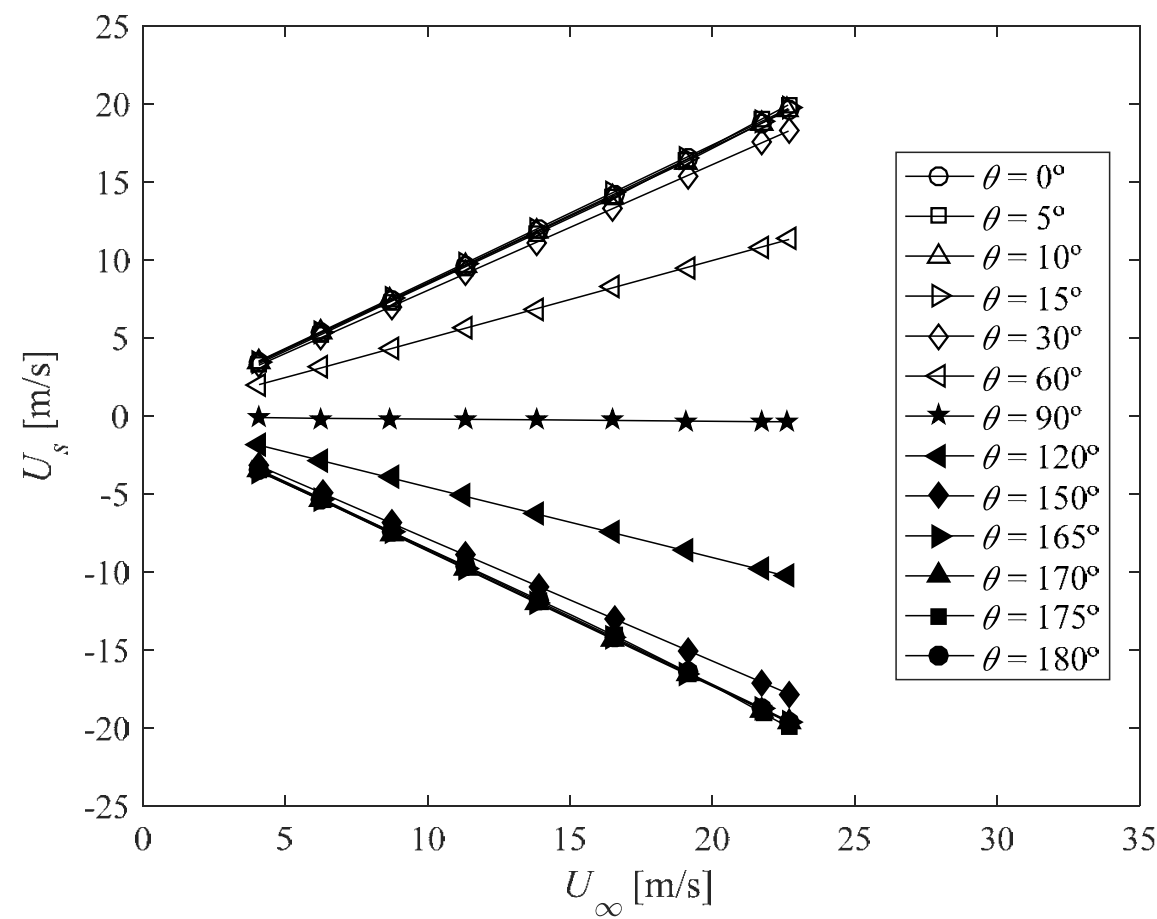

Fig. 6.1: Wind speed measured by the UA based on the free stream wind speeds for different stationary azimuthal angles.

Indeed, the velocity measurements for the azimuthal angles from $90^{\circ}$ to $180^{\circ}$ are negative. In these cases, the transmitter is placed in the downstream of the receiver so that the forward pulse from transmitter to receiver travels against the incoming flow which takes more time comparing to backward pulse from reverse direction. So, the velocity measurements at these angles are recorded with the negative values. It is reminded from the Eq. (3.2): $\left(\left(t_{+}>t_{-} \rightarrow\left(1 / t_{+}<1 / t_{-}\right) \rightarrow\left(1 / t_{+}-1 / t_{-}\right)<0 \rightarrow U_{\mathrm{s}}=(L / 2) \times\left(1 / t_{+-}-1 / t_{-}\right) \rightarrow\right.\right.$ $\left.U_{\mathrm{s}}<0\right)$.

Fig. 6.2 shows the mean flow velocity measured by the UA, $U_{s}$, as a function of the reference velocity (mean wind tunnel flow velocity component along the sonic path), $U_{r}=U_{\infty} \cos \theta$, for all the tested azimuthal angles, $\theta$. 


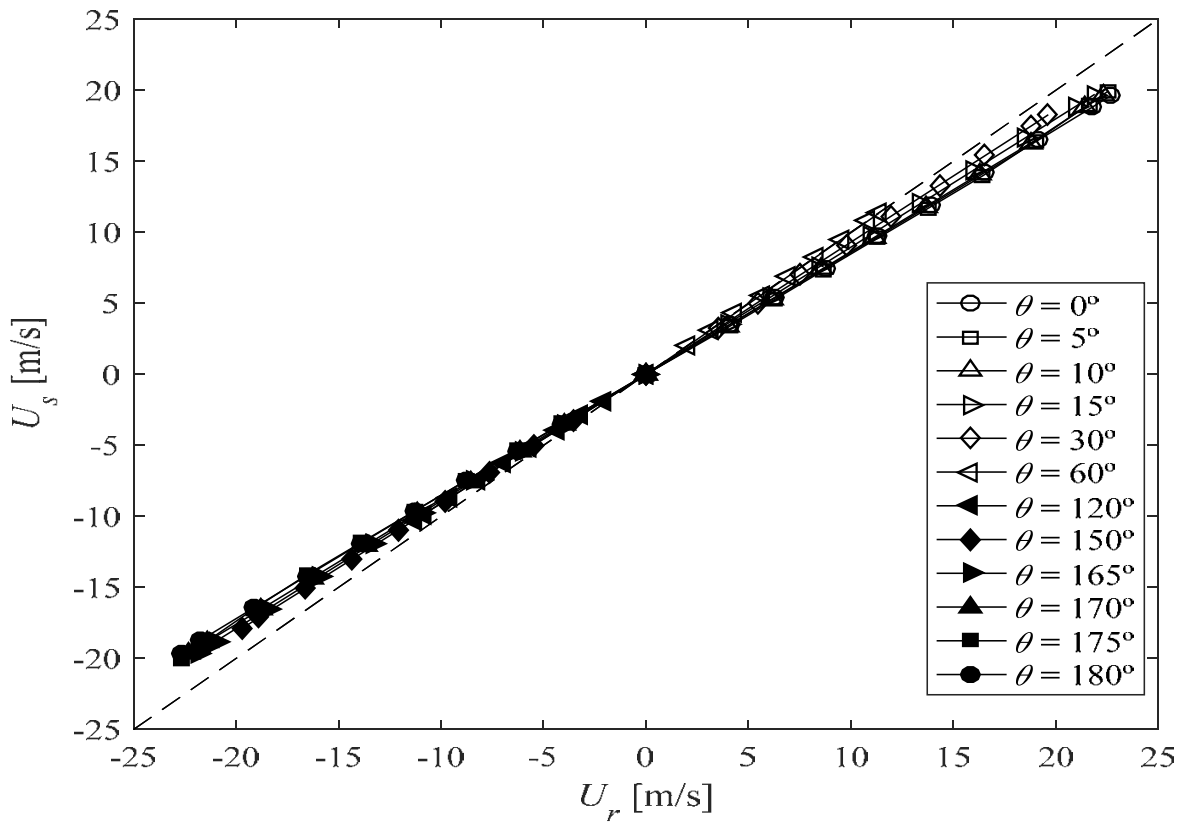

Fig. 6.2: Ultrasonic velocity measurements, $U_{s}$, as a function of the reference velocity, $U_{r}$, for different stationary azimuthal angles, $\theta$.

As expected, a linear relationship is observed between these two magnitudes. Nevertheless, the UA readings exhibit a certain deficit comparing to the reference velocity.

Shown in Fig. 6.3 is the velocity deficit, defined as $\Delta U=\left(U_{s} / U_{r}-1\right)$, as a function of the absolute value of the reference velocity, $\left|U_{r}\right|$, for the tested azimuthal angles examined. And the mean velocity deficit obtained for each reference velocity, as a function of the azimuthal angle of the ultrasonic path is shown in Fig. 6.4. As can be observed, for the studied range of wind speeds, $\Delta U$ is almost independent of the wind speed value (except in the case of $\theta= \pm 5^{\circ}$ ), while strongly depends on the orientation of the sonic path. It is seen that the velocity deficit is greater when the ultrasonic path is aligned or almost aligned with the flow.

As the misalignment increases, the wake of the upstream transducer is projected out of the ultrasonic path, reducing the distortion that occurs in the measurements. 


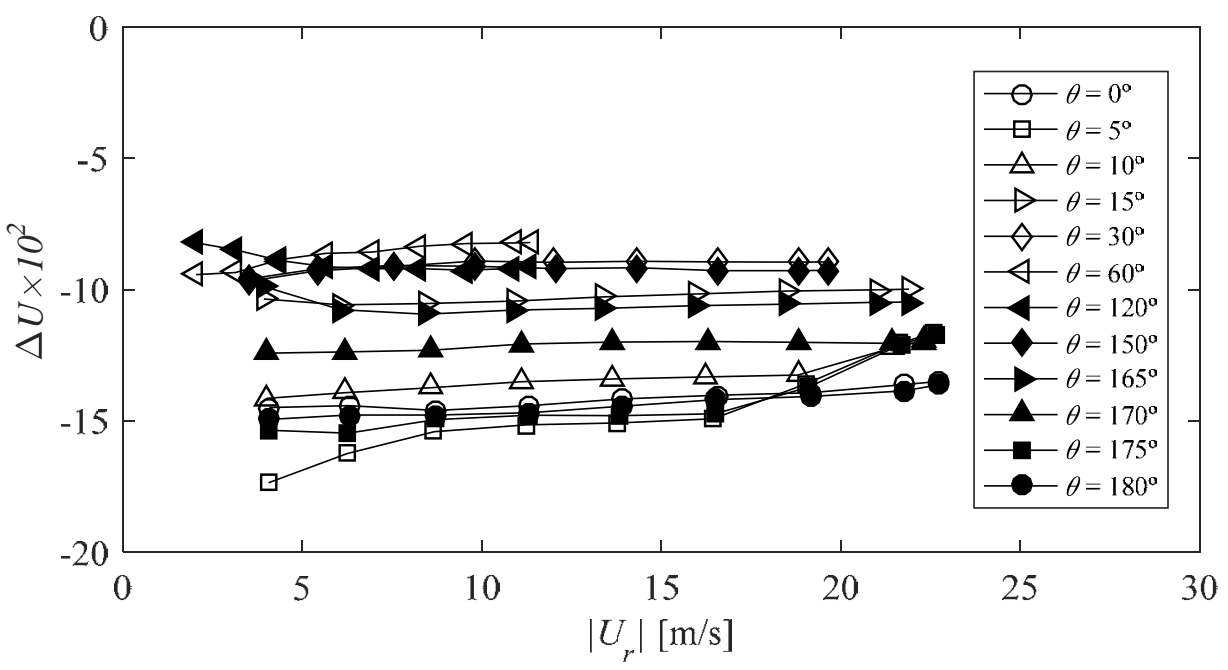

Fig. 6.3: Velocity deficit, $\Delta U$, as a function of the absolute value of the reference velocity, $\left|U_{r}\right|$.

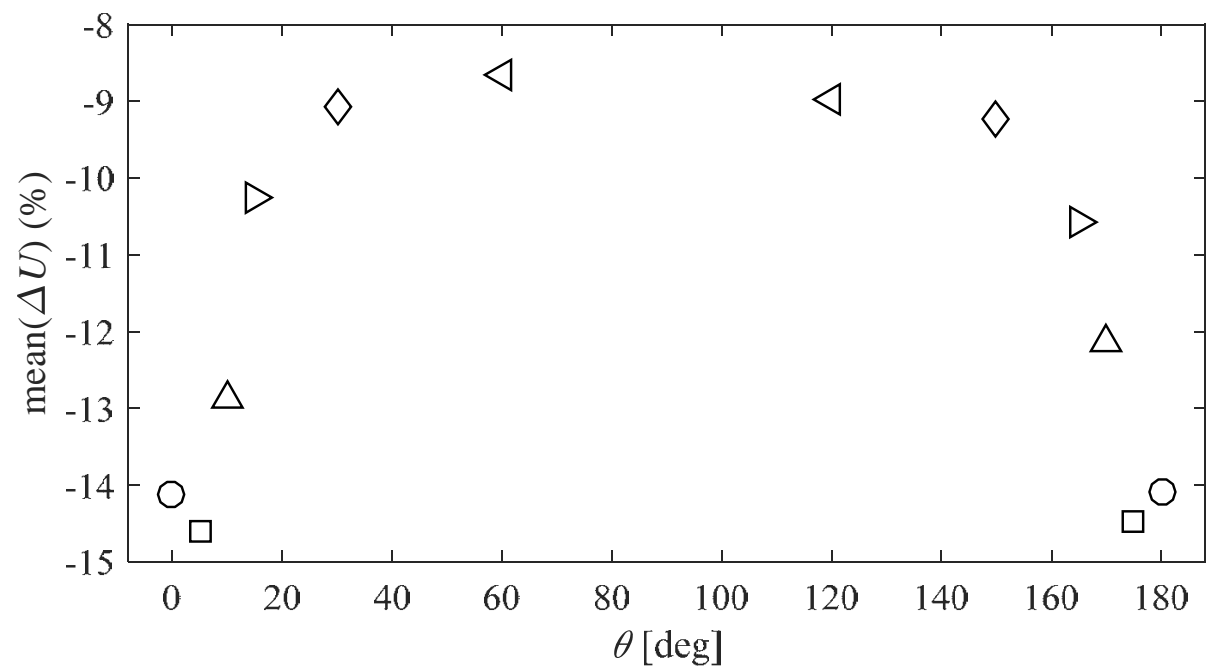

Fig. 6.4: Mean velocity deficit obtained for each reference velocity as a function of the azimuthal angle of the ultrasonic path, $\theta$.

Fig. 6.5 illustrates the standard deviation, $\sigma$, of the wind speed measured by the UA for different stationary angles between signal path and wind direction. The standard deviation in the tests show a dramatic increase at $\theta=5^{\circ}$ and $\theta=175^{\circ}$ for high wind speeds. It can be reasoned that for these critical angles the upstream sensor is slightly deviated towards the wind direction so that its cross section faced up with the incoming flow increases, and therefore stronger wakes and vortices are shed into the measurement path. 
This underscores the role of the aerodynamic design and geometric dimensions in reducing the head shadow effect on the signal path.

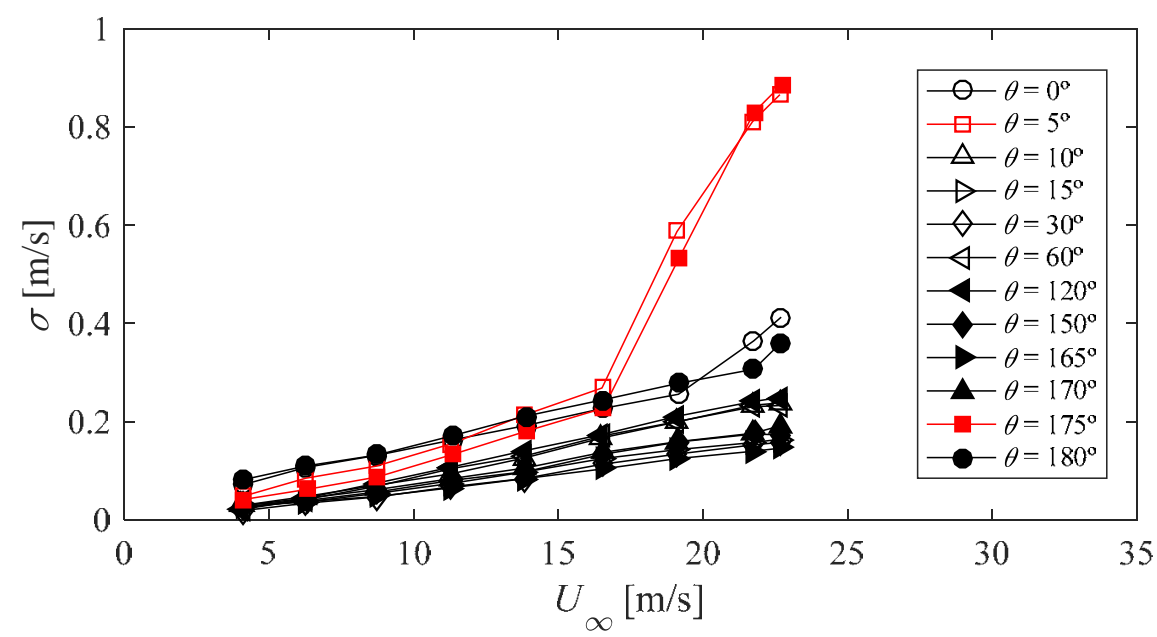

Fig. 6.5: Standard deviation of the ultrasonic velocity measurements, $\sigma$, as a function of the free wind speeds, $U_{\infty}$.

\subsubsection{Calibration for static tests}

Some semi-empirical models have already been proposed to correct the errors due to the aerodynamic interference effects of the sensor heads in UA wind speed measurements $[62,67]$. However, it is not possible to completely eliminate the sensor head shadow effect and the best way to reduce the uncertainty in the measurements, caused by the sensor shadow effect, is still to calibrate the anemometer.

The relationship between the wind reference velocities, projections of the incoming wind onto the signal path, and the UA indicated wind speed can be expressed as a calibration function:

$$
U_{r}=A U_{s}+B
$$

Where, $A$, and $B$, are the calibration coefficients which depend on the azimuthal angle of the sonic path with respect to the wind direction. The values of $A$ and $B$ are obtained by the least squares regression method and are presented in Table 8. Also, the coefficient of determination, $R^{2}$, is included as a measure of the goodness of fitting. 
Table 8: Calibration coefficients, $A$, and $B$, for different stationary azimuthal angles, $\theta$, and coefficient of determination of the fitting, $R^{2}$.

\begin{tabular}{cccc}
\hline$\theta\left[^{\circ}\right]$ & $A$ & $B[\mathrm{~m} / \mathrm{s}]$ & $R^{2}$ \\
\hline 0 & 1.1531 & 0.1094 & 1.0000 \\
5 & 1.1202 & 0.4747 & 0.9996 \\
10 & 1.1331 & 0.1891 & 1.0000 \\
15 & 1.1088 & 0.0534 & 1.0000 \\
30 & 1.0729 & 0.0252 & 1.0000 \\
60 & 1.0854 & 0.0415 & 1.0000 \\
120 & 1.1048 & 0.0283 & 1.0000 \\
150 & 1.0993 & 0.0185 & 1.0000 \\
165 & 1.1184 & -0.0375 & 1.0000 \\
170 & 1.1346 & -0.0222 & 1.0000 \\
175 & 1.1241 & -0.3674 & 0.9996 \\
180 & 1.156 & -0.1325 & 1.0000 \\
\hline
\end{tabular}

\subsubsection{Phase angle in static tests}

The reference velocity, $U_{r}$, is defined as the projection of free stream wind vector at each static azimuthal angle, $U_{r}=U_{\infty} \cos \theta$, which is considered as the ideal response of the UA in each signal path direction. As it is seen in Fig. 6.6 (left), the velocity measured by UA has discrepancy with the reference velocity certainly in the shadow regions where the signal path is aligned or almost aligned with flow direction.
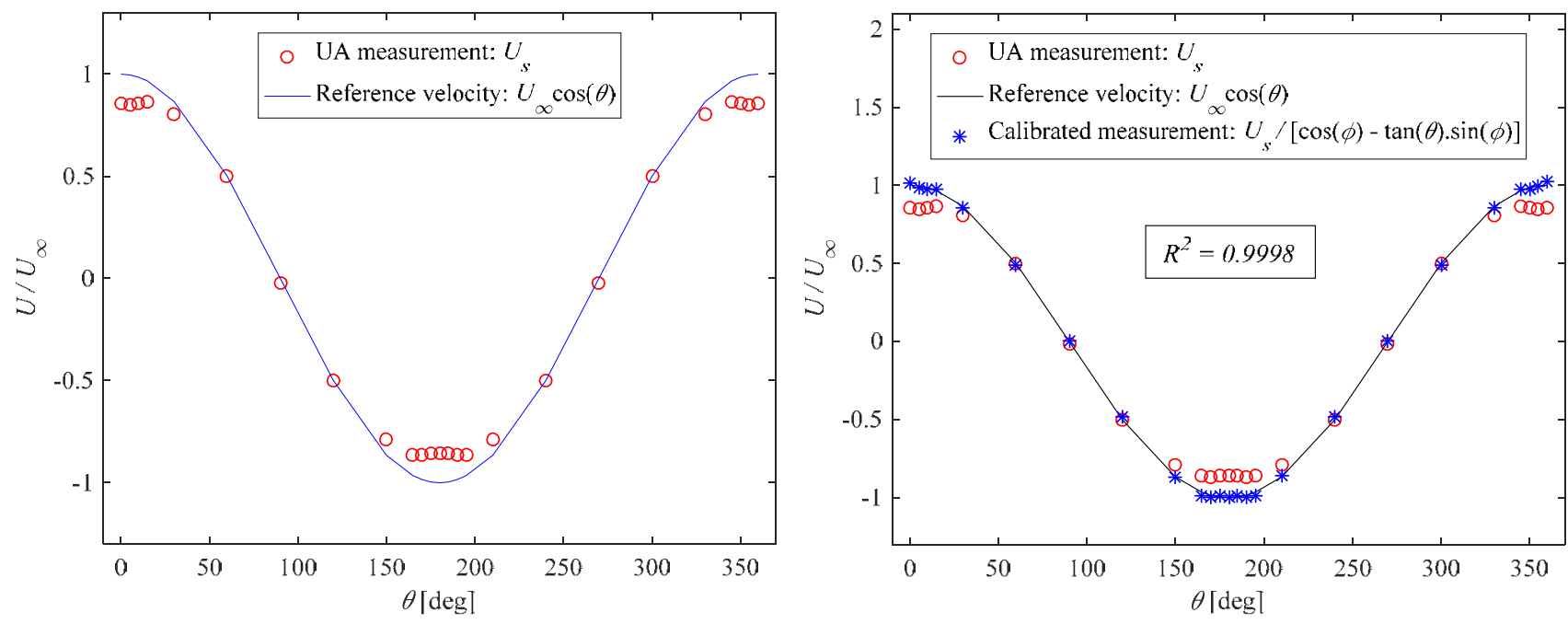

Fig. 6.6: UA measurements and the reference velocity (left), and the performance of the calibrated model (right). 
This confirms that the uncertainty in the UA measurements depends on the orientation of the signal path. Here, the sensor head distortion effect is considered to be in the form of a phase shift or lag into the azimuthal angle of the signal path. This is the major source of the discrepancies observed between the UA measurement and that expected values. A phase angle, $\phi$, is thus added to the azimuthal angle of the signal path, as introduced in Eq. (6.2).

$$
U_{s}=U_{\infty} \times \cos (\theta+\phi)
$$

At each azimuthal angle, $\theta(i)$, for different incoming wind speeds, $U_{\infty}(j)$, and ultrasonic velocity measurements, $U_{s}(j)$, a ‘ $\phi(j)$ ' can be calculated by Eq. (6.3):

$$
\phi(j)=\arccos \left(\frac{U_{s}(j)}{U_{\infty}(j)}\right)-\theta(i)
$$

where $i=1, \ldots, 13$, and $j=1, \ldots, 9$, therefore, for the 9 different wind speeds that were set at each azimuthal angle, $\theta(i)$, a mean phase angle, $\phi(i)$, is calculated by:

$$
\phi(i)=\frac{\sum_{j=1}^{9} \phi(j)}{9}
$$

The mean phase angle, $\phi$, has been calculated for all the azimuthal angles fixed in the static tests and a regression polynomial was fitted on them, as shown in Fig. 6.7.

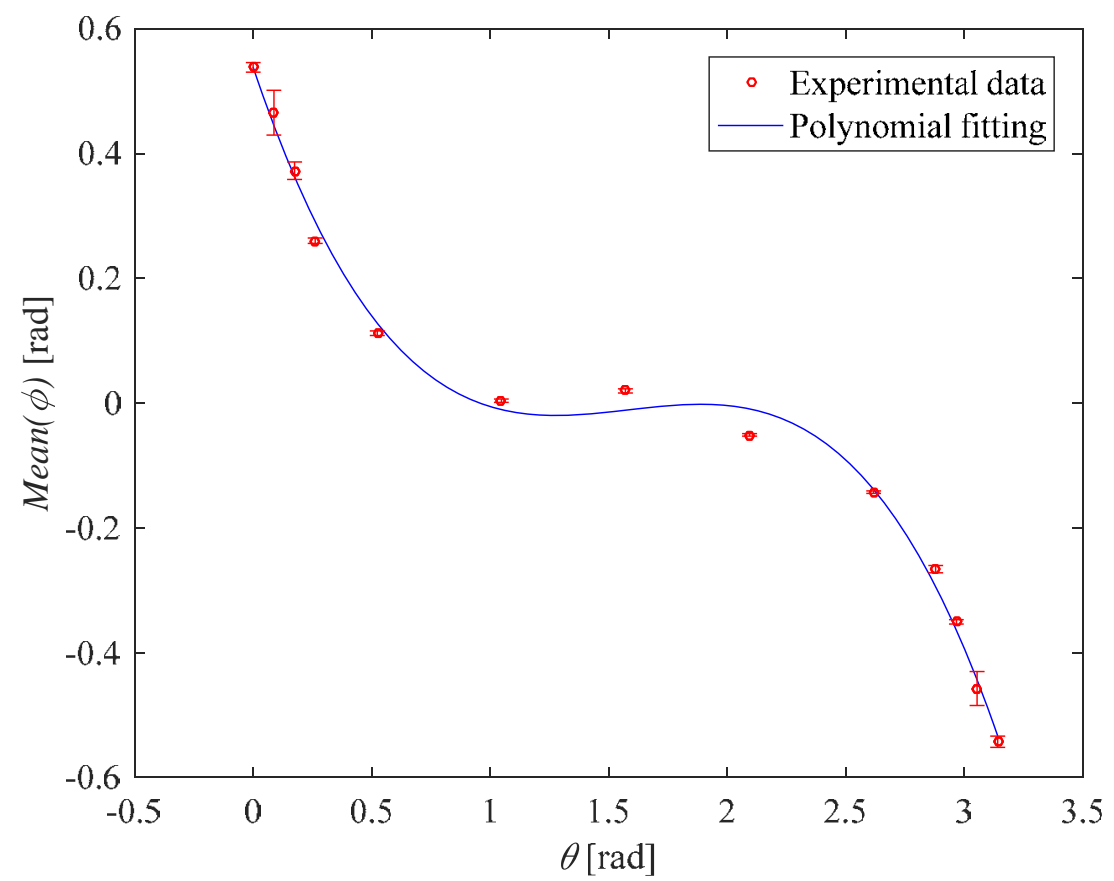

Fig. 6.7: Phase angle, $\phi$, as a function of the tested azimuthal angle, $0<\theta^{\circ}<180$. 
As it is seen from the error-bar, a higher difference between minimum and maximum values of the phase angles $(\phi)$ are observed for the azimuthal angles of $5^{\circ}$ and $175^{\circ}$. This is because of the larger transducer head distortions into the measurement path at these critical angles, as discussed earlier.

Here, the phase angle, $\phi$, is calculated by a third order polynomial function of the tested azimuthal angle, $\theta$, as it is given in the Eq. (6.5):

$$
\phi=-0.1560 \theta^{3}+0.7406 \theta^{2}-1.1284 \theta+0.5381
$$

Eq. (6.5) works for $0<\theta<\pi$, in order to find a phase angle function which works for a complete revolution of the UA signal path with wind direction, the measurements from the static tests for $0<\theta<2 \pi$ should be evaluated. Hence, due to the symmetrical position of each stationary azimuthal angle with the longitude axis of the test section, the UA wind speed measurement at each azimuthal angle from 0 to $\pi / 2$ would be equal to the measurement at its symmetric azimuthal angle from $3 \pi / 2$ to $2 \pi$. Similarly, the UA measurements for azimuthal angles from $\pi$ to $3 \pi / 2$ are obtained from the measurements in their symmetrical positions from $\pi / 2$ to $\pi$. On this basis, the mean phase angle for the azimuthal angles from 0 to $2 \pi$ can be calculated by Eq. (6.3) and Eq. (6.4). In Fig. 6.8, the calculated mean phase angle for the azimuthal angles from 0 to $2 \pi$ along with its polynomial fitting are illustrated.

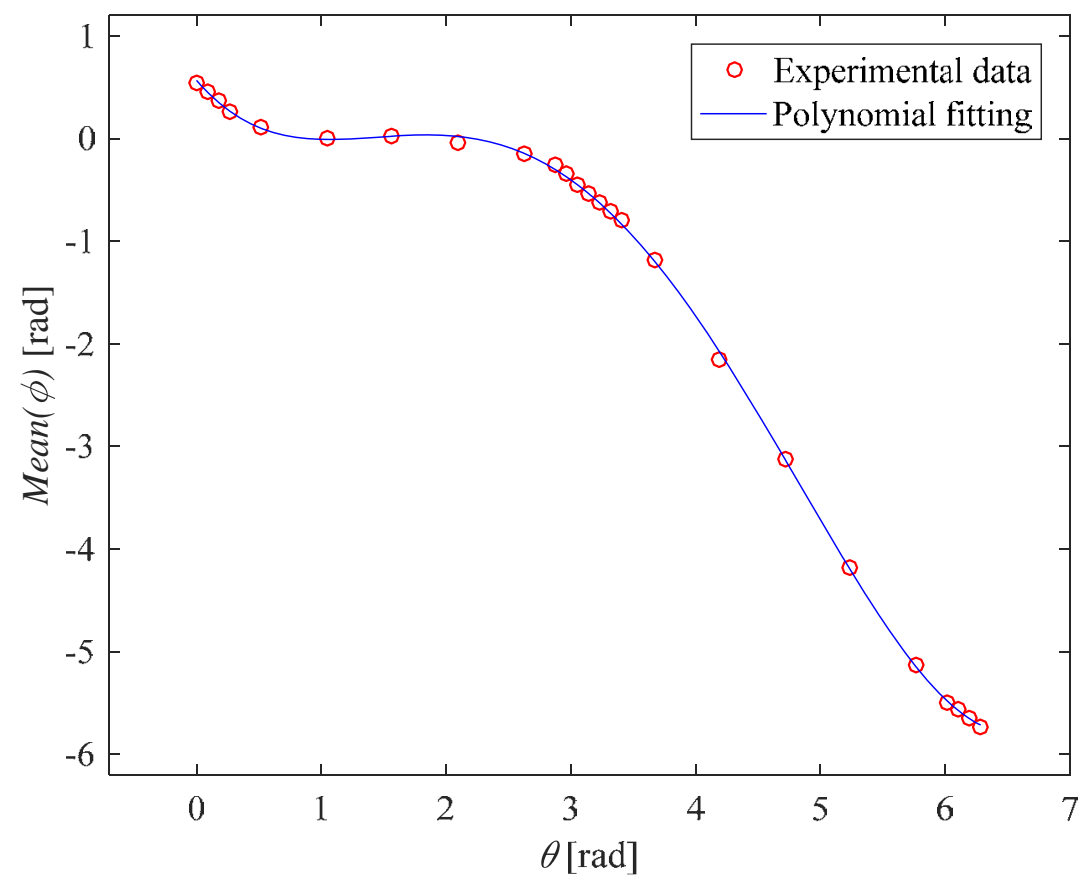

Fig. 6.8: Phase angle, $\phi$, as a function of the azimuthal angle, $0<\theta^{\circ}<360$. 
Therefore, the phase angle, $\phi$, for each UA signal path orientation, $0<\theta<2 \pi$, can be obtained by a fourth-order polynomial function as shown in Eq. (6.6):

$$
\phi=0.0275 \theta^{4}-0.3456 \theta^{3}+1.1507 \theta^{2}-1.4069 \theta+0.5661
$$

The calculated phase angle from Eq. (6.6) is substituted into Eq. (6.2), to obtain Eq. (6.7).

$$
\begin{gathered}
U_{S}=U_{\infty} \times \cos (\theta+\phi)=U_{\infty}[\cos (\theta) \cos (\phi)-\sin (\theta) \sin (\phi)] \\
=U_{\infty} \cos (\theta)[\cos (\phi)-\tan (\theta) \sin (\phi)]
\end{gathered}
$$

The calibrated measurement, $U_{c}$, is supposed to be equal to the reference velocity, $U_{r}$. The calibration function can thus be derived in Eq. (6.8):

$$
U_{c}=U_{\infty} \cos (\theta)=\frac{U_{s}}{\cos (\phi)-\tan (\theta) \sin (\phi)}
$$

where $U_{s}$ is the velocity measured by UA, $\theta$ is the azimuthal angle between UA signal path with wind direction, and $\phi$ is the phase angle as a function of $\theta$.

Shown in Fig. 6.6 (right), the calibrated values $\left(U_{c}\right)$ are in a good agreement with the reference velocities $\left(U_{r}\right)$ at different stationary azimuthal angles.

\subsubsection{CFD Simulations}

The wind-tunnel test section and UA are simulated in Ansys-Fluent and the flow-field wind speed is calculated for different stationary azimuthal angles of the signal path. The CFD results also shows a discrepancy between the free stream wind speed and the velocity calculated in the transducer's shadow regions where the measurements are affected by the transducers' wakes and distortions.

According to the results, the wind speeds calculated with CFD and experimental tests for different stationary azimuthal angles of the signal bath are in a very good agreement. Therefore, it is concluded that the transducer shadow effect is the major source of uncertainty in the ultrasonic wind speed measurements.

Since the CFD calculations is not defined in the objectives of this thesis, a short description on how to simulate the static tests and CFD calculations by Ansys-Fluent are described in the Appendix B for the interested readers. 


\subsection{Dynamic tests}

The outputs of the dynamic tests are the wind velocity measured by the UA, $U_{s}(t)$, the instantaneous orientation of the signal path regarding the wind-tunnel flow, $\theta(t)$, and the reference wind-tunnel flow velocities, $U_{\infty}$, measured by pitot-tube. Since all the measurements were recorded simultaneously with the same time base, it is possible to analyze the ultrasonic measurements as a function of its orientation.

The test plan consisted of 156 runs, as illustrated in Fig. 6.9 For each test a wind speed for wind tunnel, $U_{\infty}$, and a rotational speed, $\omega$, for ultrasonic anemometer were set. The rotational velocities for the UA were selected due to the acceptance working range of the slip-ring-capsule which is up to $250 \mathrm{rpm}$ or $26.17 \mathrm{rad} / \mathrm{s}$.

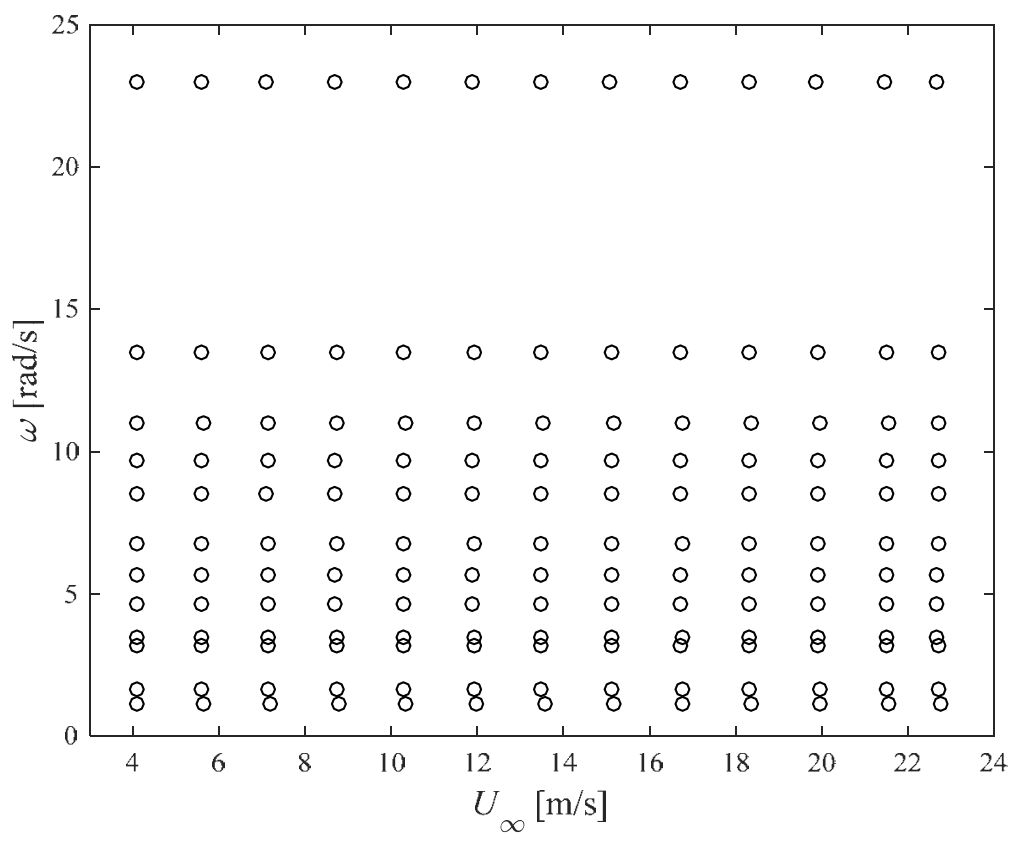

Fig. 6.9: Entire experimental tests in which every circle indicates a dynamic test.

Figures 6.10 and 6.11 show the effects of free stream velocity and UA rotational speed on variations of the UA measured velocity at different azimuthal angles, respectively. Some irregular behavior can be observed at $\theta=0,180$ and 360 degrees where the measurements are affected by the transducer head distortion. This effect gets more prominent at higher wind speeds. Furthermore, the impact of the wind velocity is evidently more pronounced than that of the rotational speed on the velocity measured by the UA. 


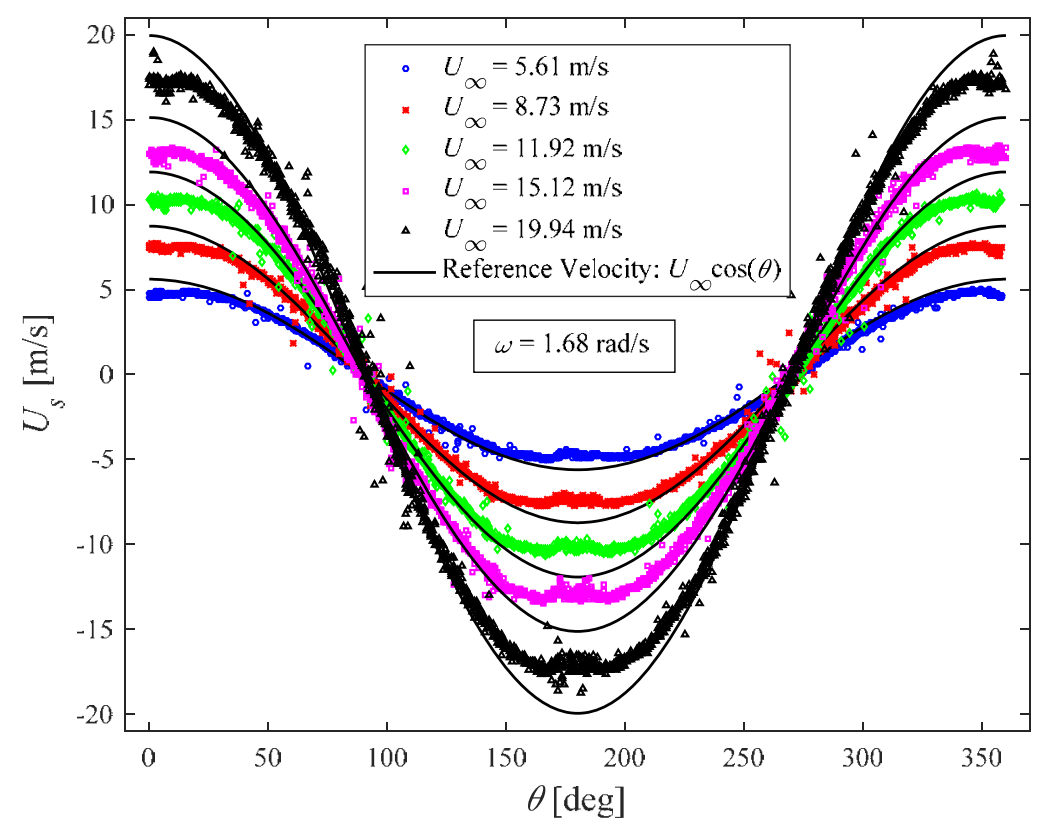

Fig. 6.10: Effects of free stream wind speed on variations of the UA measured velocity.

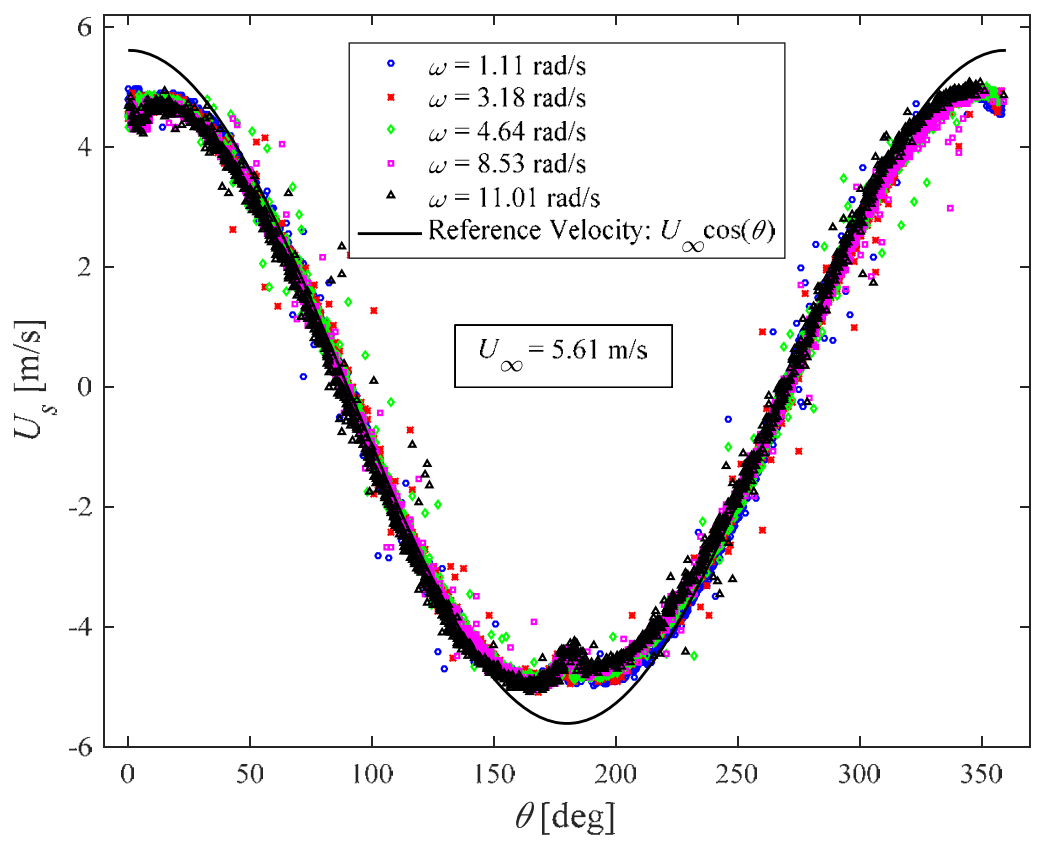

Fig. 6.11: Effects of UA rotational speed on variations of the UA measured velocity.

\subsubsection{Accuracy of measurements}

As mentioned earlier, the flow uniformity inside the section has been calculated during the wind-tunnel calibration process and was found to be better than $0.2 \%$. According to the technical specifications provided by the manufacturer, the resolution of the velocity indicated by the UA in the present experiments was $0.01 \mathrm{~m} / \mathrm{s}$. 
Basically, the instruments and systematic errors affect the output velocity signals from the UA. Fig. 6.12 shows a typical dynamic test results in which, the UA was rotating at an angular velocity of $5.69 \mathrm{rad} / \mathrm{s}$ and a wind speed of $16.73 \mathrm{~m} / \mathrm{s}$. The instantaneous velocity measured by the UA is plotted for each instantaneous azimuthal angle for 10 revolutions.

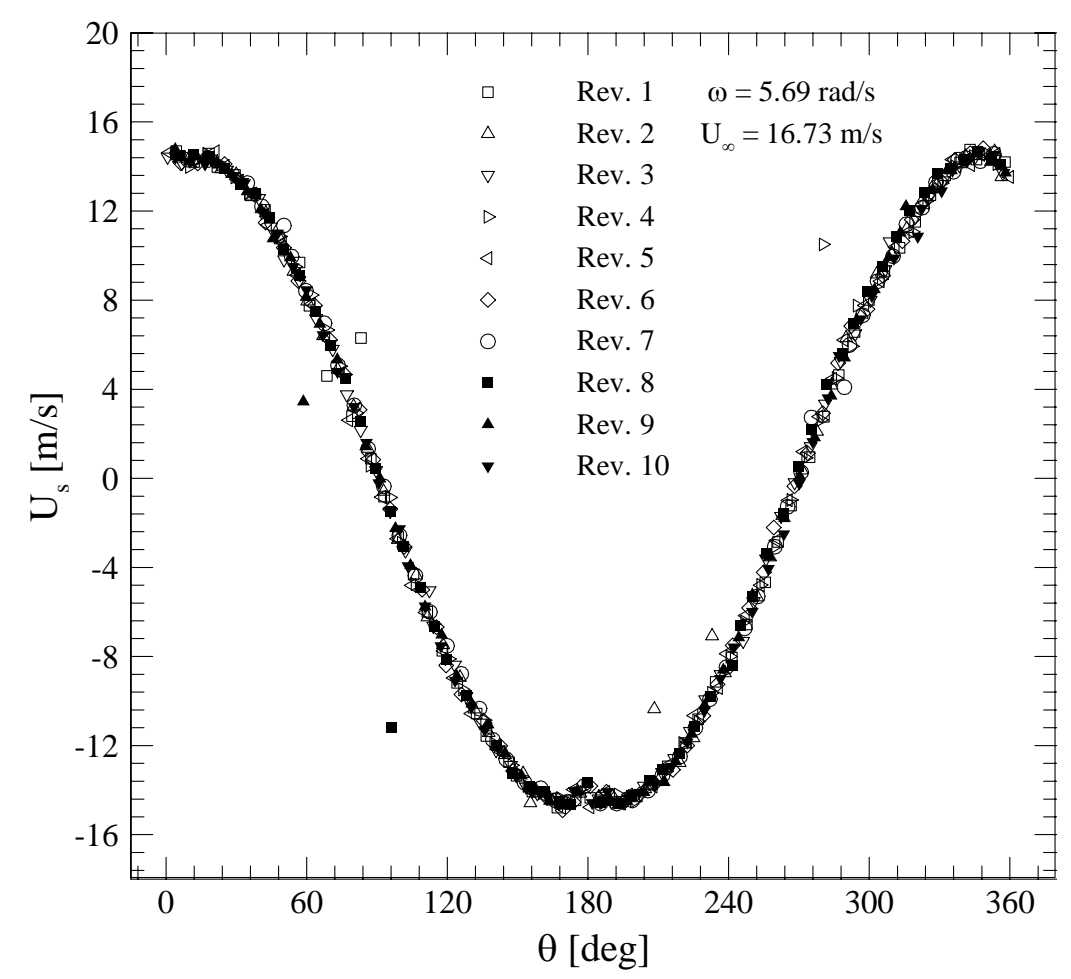

Fig. 6.12: UA indicated velocity at different instantaneous azimuthal angles for 10 revolutions.

Since the rotation is sinusoidal, the indicated velocity at different azimuthal angle is also of sinusoidal form except at the shadow regions, as discussed earlier. Good repeatability can be observed from this figure. However, some bad points can also be observed which can be due to UA reading and position sensor errors.

Nevertheless, the pattern must theoretically be symmetric and this is a criterion to check the reliability and repeatability of data. Data skewness, mean and median are the statistical parameters that quantitatively describe the overall behavior.

Skewness is the degree to which a data set is not symmetrical. As data becomes more symmetric, its skewness value approaches zero. The mean value describes an entire set of the measured velocities with a single value representing the center of the data. It is 
defined as the sum of all the measured velocities divided by the total number of samples. Median is the middle of the range of data. Half of the measured velocities are less than or equal to it and the rest are greater than or equal to it [96].

The values of these three parameters for 10 revolutions are tabulated in Table 9 for a typical spinning test with $U_{\infty}=16.73 \mathrm{~m} / \mathrm{s}$, and $\omega=5.69 \mathrm{rad} / \mathrm{s}$. Both the mean and median are nearly zero indicating that the measured velocities are symmetrically distributed between the positive and negative values corresponding to $\pi / 2<\theta<3 \pi / 2$ for negative velocities, and $3 \pi / 2<\theta<5 \pi / 2$ for positive ones. The value of the skewness is also small enough to make sure that the measured velocities are nearly symmetrical about zero mean. Histogram is a bar graph to represent the distribution of measured data [68]. The histogram of the UA measured velocity values, is shown in Fig. 6.13 and approves that the errors associated with the spinning mechanism, position sensor, and UA sensor are within acceptable range.

Table 9: Calculated statistical parameters for 10 revolutions of a typical spinning test.

\begin{tabular}{|c|c|c|}
\hline Mean & Median & Skewness \\
\hline 0.0256 & 0.1 & -0.0105 \\
\hline
\end{tabular}

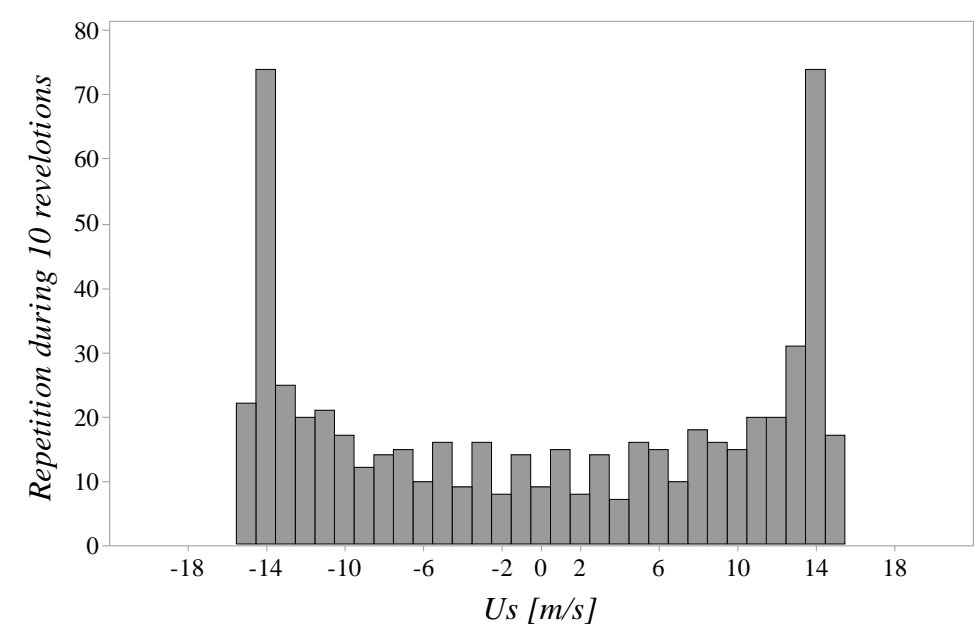

Fig. 6.13: Histogram of the UA velocity measurements during 10 revolutions.

In addition to the abovementioned uncertainty analysis, the statistical information about the instantaneous indicated velocities during 7 revolutions are tabulated in Table 10 for another typical test $\left(U_{\infty}=11.89 \mathrm{~m} / \mathrm{s}\right.$, and $\left.\omega=5.69 \mathrm{rad} / \mathrm{s}\right)$.

The mean standard error, SE Mean, measures how precisely a cycle mean velocity estimates the mean velocity of all cycles, and is used to create confidence intervals to perform an uncertainty analysis. This parameter has been calculated for each cycle as the 
standard deviation of the velocities in that cycle divided by the square root of the number of measurements [68]. The rather low value for the mean standard error in each cycle, as tabulated, shows that the overall mean has been estimated with a good accuracy. The Mean and Standard deviation values for all of the 7 cycles examined here are nearly identical. This approves the repeatability of the results, and indicates that the data acquired during each revolution can be re-produced within an acceptable accuracy range. Table 10 shows that the skewness value for each revolution is very small, indicating that the measured velocities are normally distributed. A normal distribution of data guarantees that there is little or no systematic error in the instrumentations and measurement processes. Thus, most of the errors encountered in the present experiments can be deemed to be of random type.

Table 10: Statistical parameters of the indicated velocities during 7 revolutions for a typical test with $U_{\infty}=11.89 \mathrm{~m} / \mathrm{s}$, and $\omega=5.69 \mathrm{rad} / \mathrm{s}$.

\begin{tabular}{cccc}
\hline Cycle & SE Mean & Skewness & Standard Dev \\
\hline $\mathbf{1}$ & 1.04 & -0.01 & 7.78 \\
$\mathbf{2}$ & 1.04 & 0.01 & 7.69 \\
$\mathbf{3}$ & 1.04 & 0.01 & 7.77 \\
$\mathbf{4}$ & 1.06 & 0.06 & 7.79 \\
$\mathbf{5}$ & 1.06 & 0.01 & 7.82 \\
$\mathbf{6}$ & 1.08 & 0.09 & 7.87 \\
$\mathbf{7}$ & 1.04 & 0.03 & 7.75 \\
\hline
\end{tabular}

Shown in Fig. 6.14 (a, and b) is the histogram plot of the velocity measured by the spinning UA during two different revolutions as a sample for a typical test. This plot shows how frequent each value of velocity has been repeated during a revolution. According to Fig. 6.15, the measured velocity for each revolution of UA exhibits a normal and symmetric distribution with nearly zero mean. This makes sure that the errors encountered in the present problem are mostly of random type. 

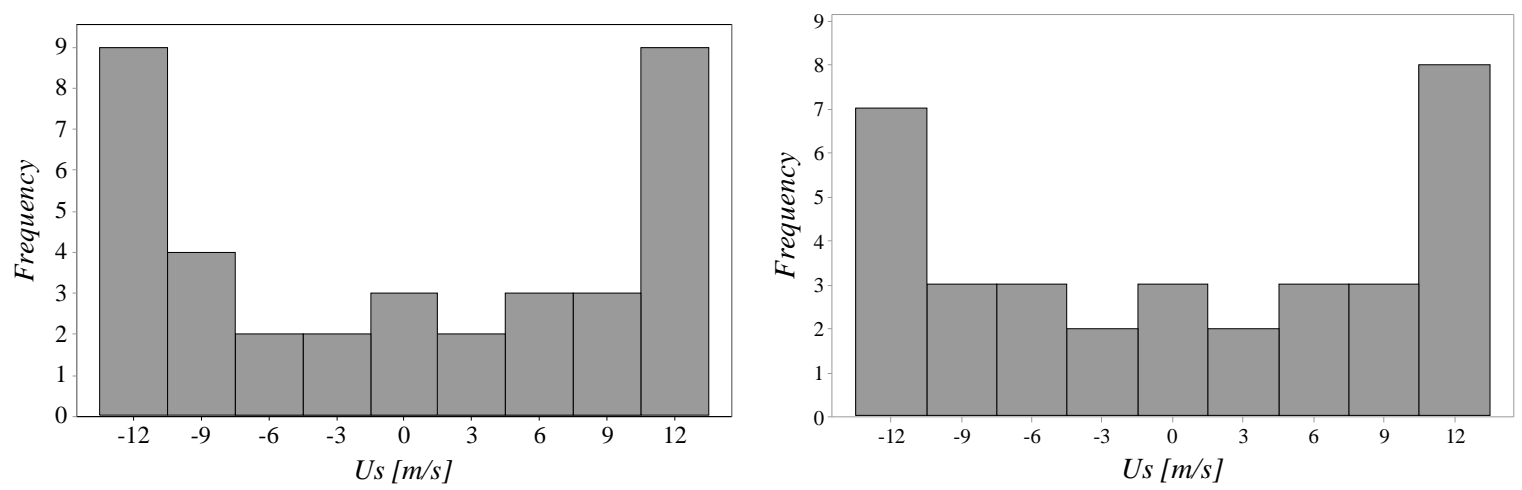

Fig. 6.14: Histogram of the velocity measured by the spinning UA for two cycles of a typical test ( $U_{\infty}=15.10 \mathrm{~m} / \mathrm{s}$ and $\left.\omega=8.53 \mathrm{rad} / \mathrm{s}\right)$ : (a) the $15^{\text {th }}$ revolution, (b) the $35^{\text {th }}$ revolution.

Systematic errors, in contrast, tend to have a sudden onset, since they're often due to a machine or process failure. They often tend to be on a larger scale than random errors and always occur as either high or low, rather than as a mixture of the two. In the present experiments, the UA itself and the other measuring devices such as the potentiometer have been checked in the pre-test calibration process and show a negligible systematic error.

For each combination of the wind speed and the angular velocity, the uncertainty in velocity signals measured by the UA was determined by calculating the pooled standard deviation for all cycles, which is a weighted average of each cycle's standard deviation [68] and calculated by:

$$
\sigma_{\text {pooled }}=\sqrt{\frac{\left(\mathrm{n}_{1}-1\right) \sigma_{1}^{2}+\left(\mathrm{n}_{2}-1\right) \sigma_{2}^{2}+\cdots+\left(\mathrm{n}_{\mathrm{k}}-1\right) \sigma_{\mathrm{k}}^{2}}{\mathrm{n}_{1}+\mathrm{n}_{2}+\cdots+\mathrm{n}_{\mathrm{k}}}}
$$

where $k$ is the point number, $n$ is the number of recorded samples at each point, and $\sigma$ is the standard deviation of $n$ samples at each point. For instance, for a typical test with $U_{\infty}=16.73 \mathrm{~m} / \mathrm{s}$ and $\omega=3.18 \mathrm{rad} / \mathrm{s}$, the statistical data (just for the first 8 points, $k=1,2, \ldots$, 8) are presented in Table 11. Therefore, for this case, the pooled standard deviation is calculated with Eq. (6.9) as 0.44 for the whole 360 points. On this basis the Fig. 6.15 shows the data uncertainty for the typical case treated earlier. 
Table 11: Statistical data for a typical test with $U_{\infty}=16.73 \mathrm{~m} / \mathrm{s}$ and $\omega=3.18 \mathrm{rad} / \mathrm{s}$.

\begin{tabular}{|c|c|c|c|c|}
\hline $\begin{array}{c}\text { Point } \\
\text { number } \\
(k)\end{array}$ & $\begin{array}{c}\text { Azimuthal } \\
\text { angle } \\
\left(\theta^{\circ}\right)\end{array}$ & $\begin{array}{c}\text { Number of } \\
\text { samples } \\
(n)\end{array}$ & $\begin{array}{c}\text { Mean velocity } \\
\text { measured by UA } \\
\left(U_{s}[\mathrm{~m} / \mathrm{s}]\right)\end{array}$ & $\begin{array}{c}\text { Standard } \\
\text { deviation } \\
(\sigma)\end{array}$ \\
\hline 1 & $0^{\circ}$ & 4 & 14.61 & 0.19 \\
\hline 2 & $1^{\circ}$ & 6 & 14.44 & 0.26 \\
\hline 3 & $2^{\circ}$ & 4 & 14.40 & 0.13 \\
\hline 4 & $3^{\circ}$ & 5 & 14.49 & 0.14 \\
\hline 5 & $4^{\circ}$ & 7 & 14.20 & 0.18 \\
\hline 6 & $5^{\circ}$ & 4 & 14.27 & 0.25 \\
\hline 7 & $6^{\circ}$ & 7 & 14.30 & 0.25 \\
\hline 8 & $7^{\circ}$ & 7 & 14.43 & 0.34 \\
\hline
\end{tabular}

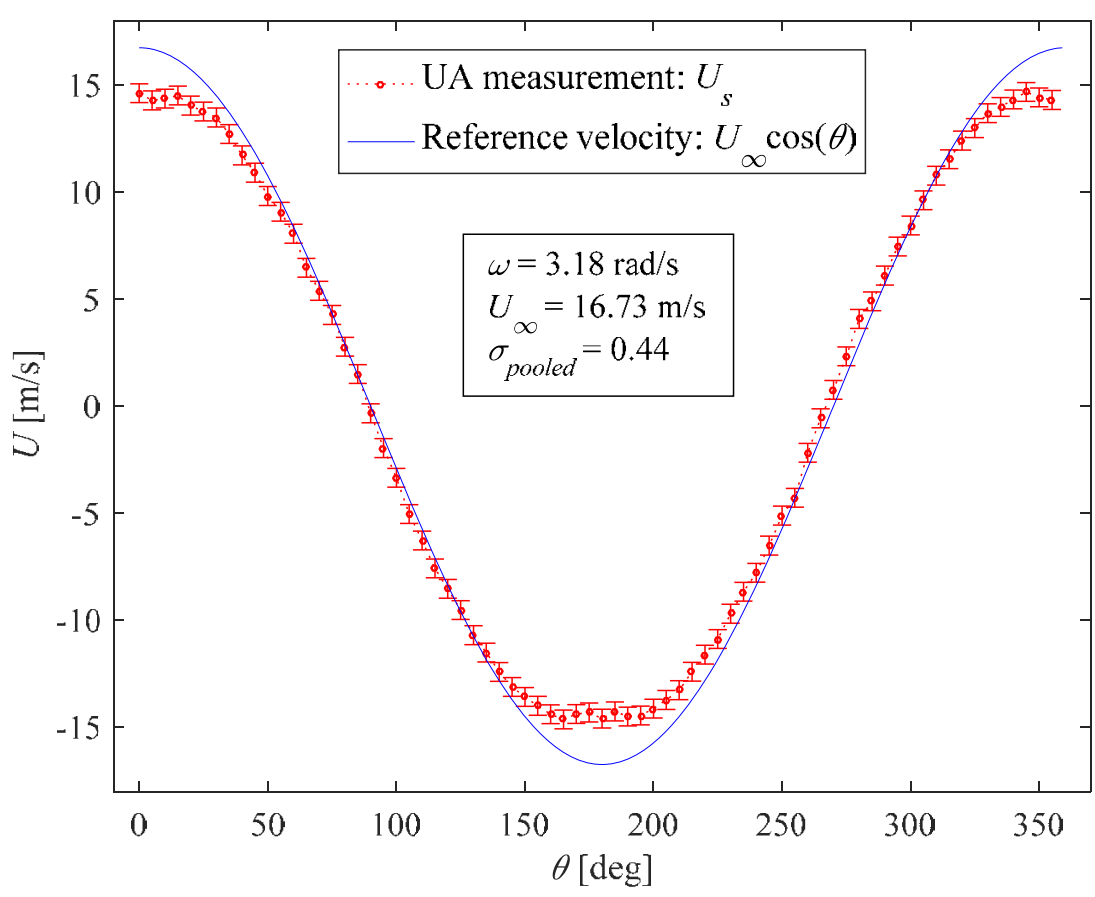

Fig. 6.15: Uncertainty measurement for a typical case.

The reference velocity is defined as the free stream wind velocity component along the UA signal path, $U_{r}=U_{\infty} \cos \theta$. The measurement accuracy is a diagnostic comparing ultrasonic velocity measurements, $U_{s}$, and reference velocities, $U_{r}$. The relative accuracy 
or velocity deficit is obtained by $\Delta U=\left(1-U_{s} / U_{r}\right)$, and illustrates for a typical test with $U_{\infty}=8.71 \mathrm{~m} / \mathrm{s}$ and $\omega=4.64 \mathrm{rad} / \mathrm{s}$ in Fig. 6.16. It is seen that at the azimuthal angles $90^{\circ}$ and $270^{\circ}$ when the signal path is perpendicular to the flow direction, the travelling times of the pulses are almost equal, $t_{-} \approx t_{+}$, and due to Eq. (3.2) the anemometer measures almost zero wind speed, i.e. $|\Delta U|=1$. Since, only noise is recorded by the sensors in such conditions, the uncertainties in the relative error considerably increases. In the transducer shadow regions, at the azimuthal angles $0^{\circ}, 180^{\circ}, 360^{\circ}$, the signal path is aligned to the flow direction so that the maximum wakes are shed into the measurement path and the velocity deficit is seen about $20 \%$.

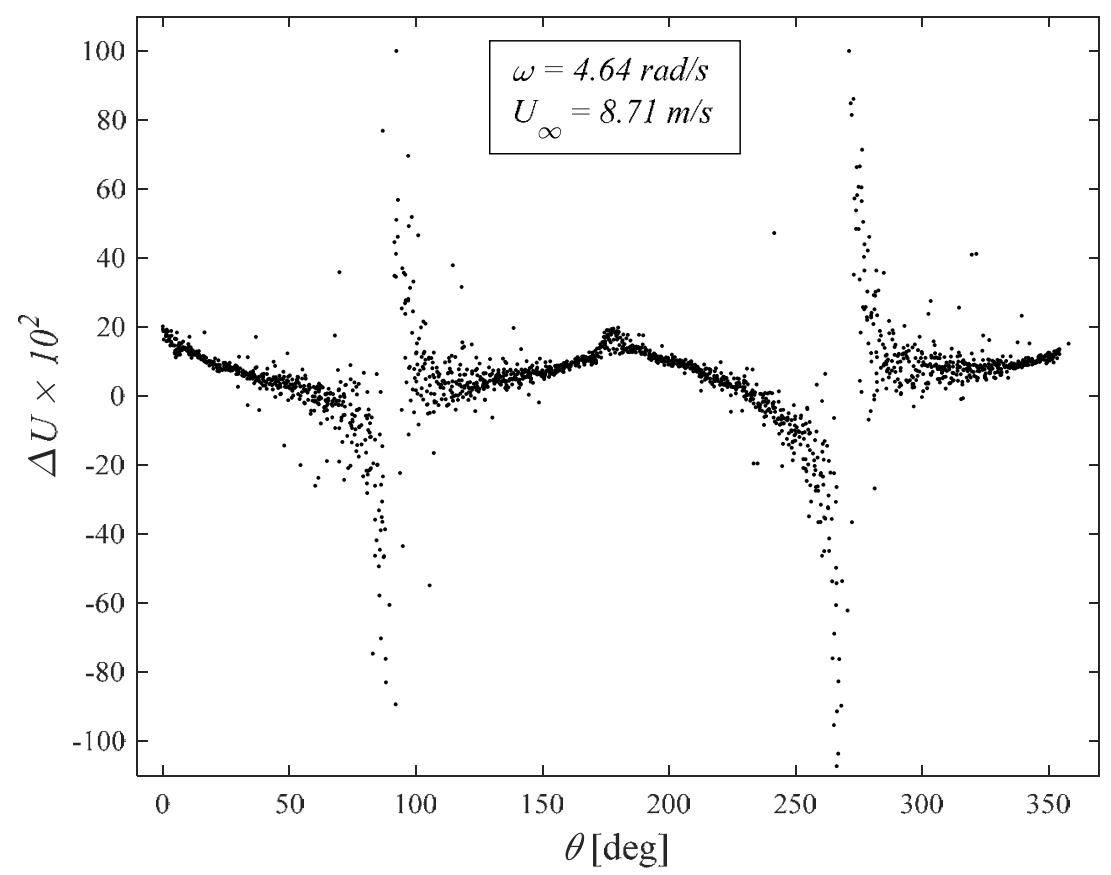

Fig. 6.16: Velocity deficit for a typical spinning test. 


\subsubsection{Phase shift in dynamic tests}

UA was rotating inside the wind-tunnel test chamber. The actual free stream velocity measured by pitot-tube, $U_{\infty}$, the wind velocity measured by sonic anemometer, $U_{s}$, and the instantaneous angular positions of the anemometer, $\theta$, have been recorded at the same time base during the tests. Output of the angular position sensor is voltage and denoted by $v$ in this literature. This voltage, according to the manufacturer's instructions, can be converted to angular position by Eq. (6.9):

$$
\theta=(v-2.5) \times 90
$$

Indeed, in each test the UA was rotating when the data-recording started. In such conditions, the velocity measurements recorded with a time lag due to the angular position measurements. This time lag or delay is observed in the form of a phase shift or misalignment in the signals measured by the UA.

Each test has its own phase shift which should be determined through the procedure below and added to the angular position data to correct for yaw misalignment. The procedure to determine the phase shift starts from Eq. (6.10). According to this equation, the position sensor in the azimuthal angle range of 90 and -90 gives a voltage between 3.5 and 1.5 , respectively which are named as ' $v_{i}$ ' onwards.

At these extreme angles, the signal path is perpendicular to the incoming flow so that the forward and backward travelling times $\left(t_{ \pm}\right)$of the acoustic pulses are almost equal and due to Eq. (3.2), the UA measures approximately zero wind speed. During the tests, the UA was rotating so that the first zero wind speed reading occurred at the azimuth angle of either 90 or -90 , depending on the sign of the ultrasonic velocity measurements, $U_{s}$. Fig. 6.17 illustrates the circular path that the transducers travel during the tests along with the sign of the velocity measured by UA at different yawing positions. 


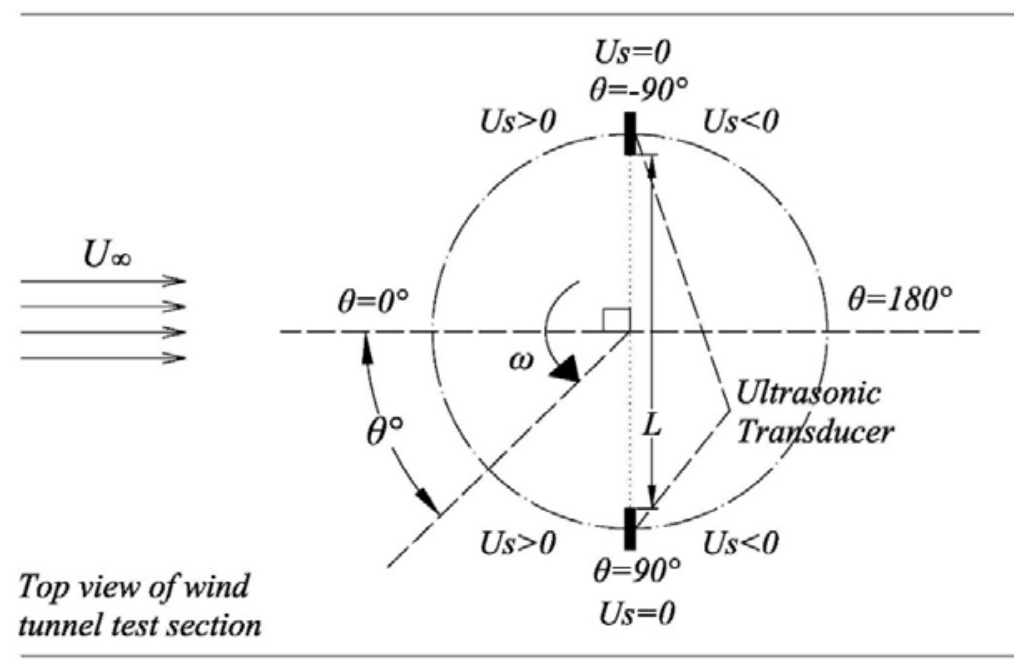

Fig. 6.17: Indication of the free stream wind and the instantaneous azimuthal angle for the spinner UA.

The voltage of the first zero indicated wind speed is then extracted from the output of the position sensor and named as ' $v e$ '. This is determined by interpolating the data in the region where the indicated speed changes sign. The phase shift is defined as ' $v_{e}-v_{i}$ ' and is designated by ' $\Delta v$ '. Expressing in terms of the instantaneous azimuthal angles, $\theta_{i}$ and $\theta_{e}$, the phase shift is obtained as: $\Delta \theta=\theta_{e^{-}} \theta_{i}=\Delta v \times 90$.

Fig. 6.18 shows the comparison of the velocity measured by the UA and the corresponding expected values for some typical tests. As can be observed there is a phase shifting in the angular positions between the measure and expected values. The expected response is $U_{\infty} \cos (\theta)$ and defined as the mean free stream wind speed component along the sonic path at each instantaneous azimuthal angle. However, some discrepancies are observed at the positions 0 and 180 degrees which are in the transducers' shadow zone.

According to Fig. 6.18 (a, b, and c), the phase shift is slightly decreased with wind speed for each rotational velocity, and the smaller phase shift is observed at a moderate UA rotational velocity. Fig. 6.18 (d) shows the impact of the rotational speed in a single plot for non-dimensional wind speeds. It is seen that the largest phase shift occurs at either low or high values of the rotational speeds, while for the moderate rotational velocities, the phase shift is observed to be minimum. 


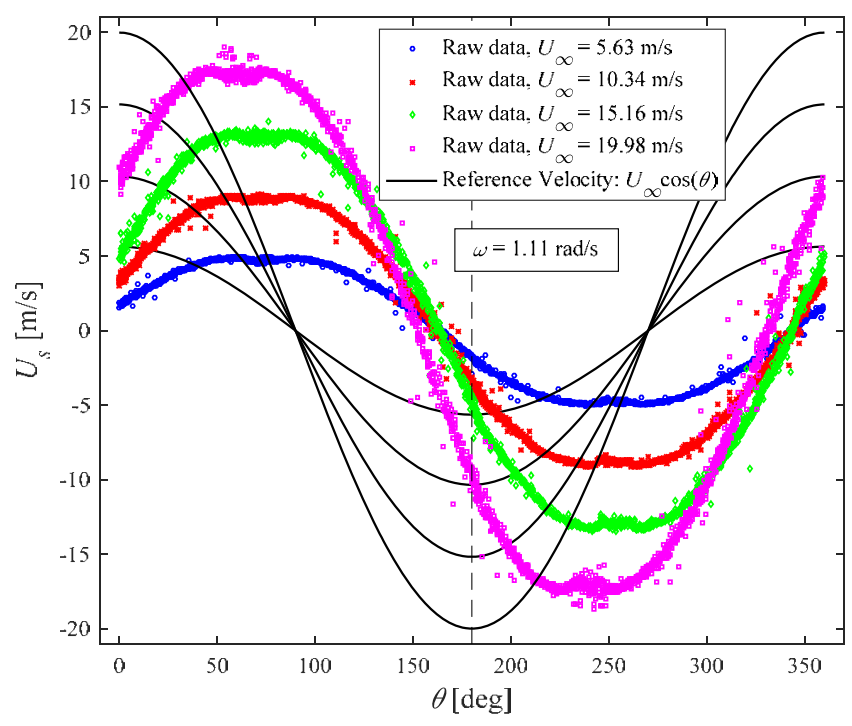

(a)

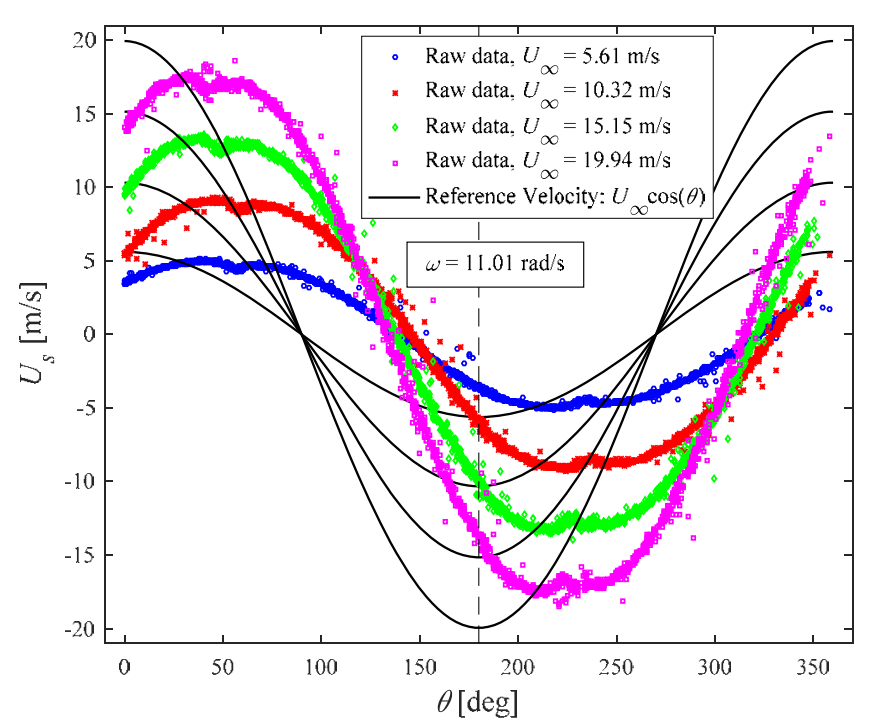

(c)

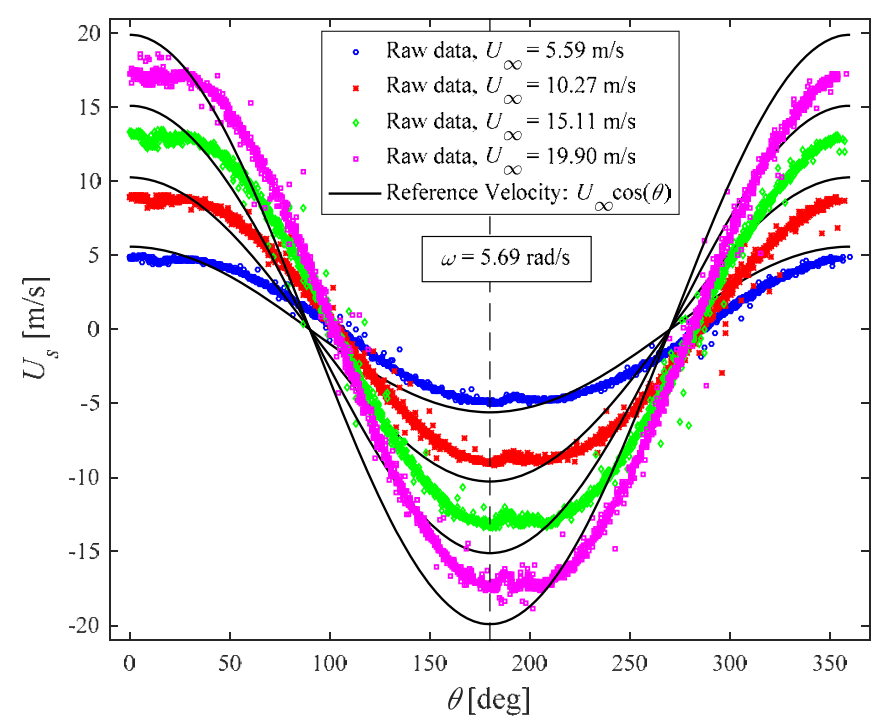

(b)

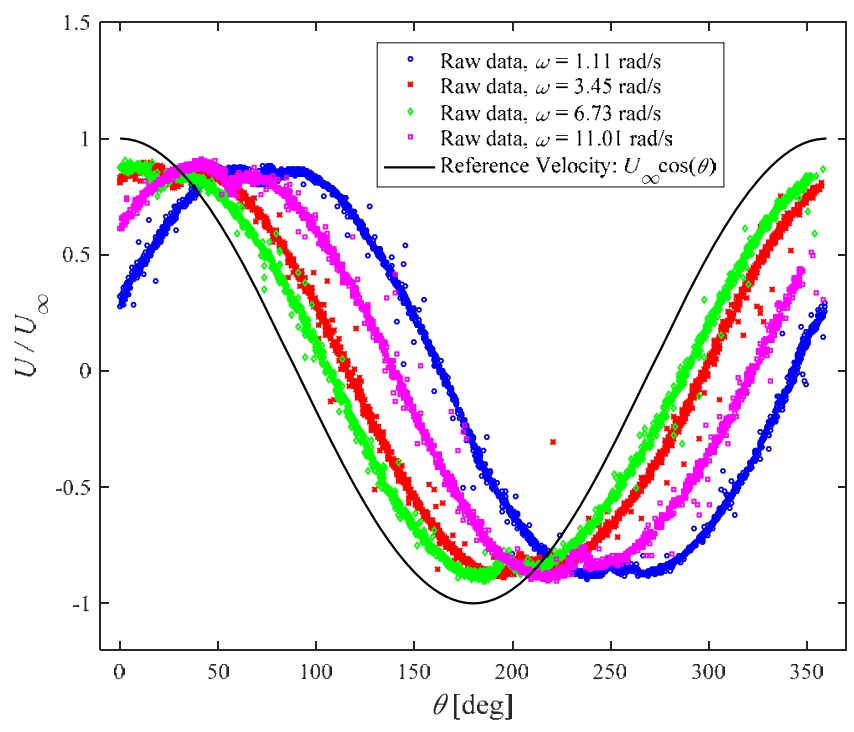

(d)

Fig. 6.18: Raw data of the velocities measured by UA for various incoming wind speeds at (a) low rotational speed, (b) moderate rotational speed, and (c) high rotational speed. And (d) non-dimensional velocity measurements for various UA rotational speeds. 
Shown in Fig. 6.19 (Left) is a monotonic behavior of the phase shift with wind speed for various UA rotational velocities. However, Fig. 6.19 (Right) illustrates remarkable fluctuations in phase shift changes with the rotational velocity for different wind speeds. According to this figure, the phase shift is nearly uniform and constant with the wind speed and is maximum for high and low rotational velocities. For a constant free stream velocity, on the other hand, the phase shift increases at the extreme values of the rotational speeds.
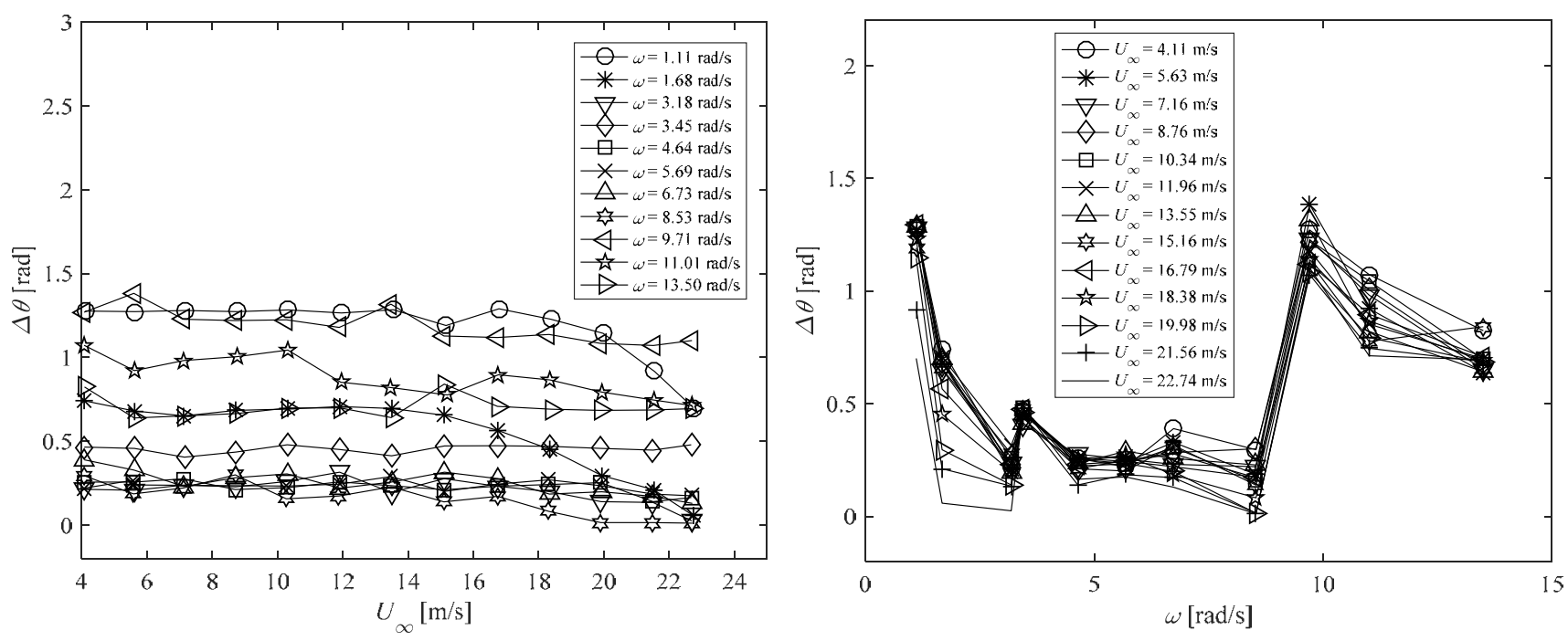

Fig. 6.19: Variation of phase shift for various UA rotational speeds (Left), and for various free stream wind speeds (Right).

The phase shift, $\Delta \theta$, is calculated for each rotational test and added into its azimuthal angle for correcting yaw misalignment in measurements. Fig. 6.20 shows the corrected measurements for phase shifting for some typical wind speeds at a UA angular velocity. It is observed that the velocity measured by the UA, once taken into account the correction for phase shift during the rotation, shows a remarkable agreement with the expected values which are $U_{\infty} \cos (\theta)$. 


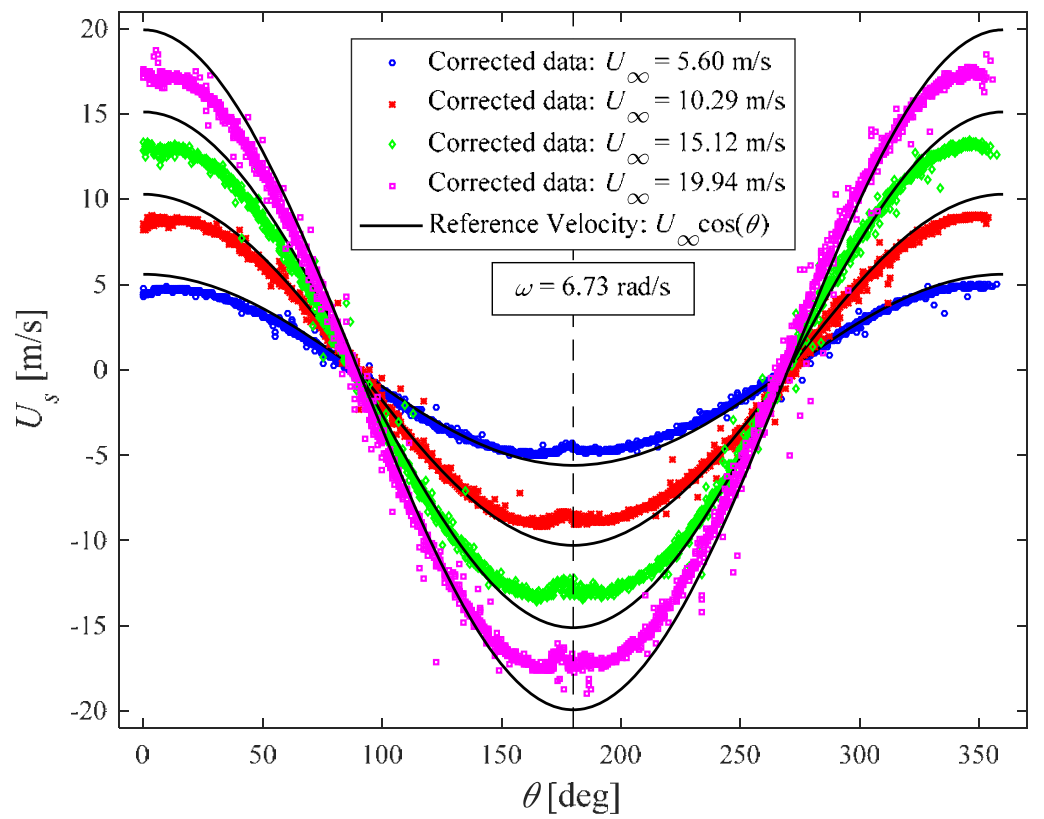

Fig. 6.20: Corrected data for yaw misalignment of the velocities measured by UA for various incoming wind speeds at a rotational speed.

\subsubsection{Calibration for dynamic tests}

In the static tests, the velocity measurements with ultrasonic anemometer were calibrated with the reference velocities. To be more exact, a linear approximation has been proposed by a first order polynomial function for correcting the ultrasonic velocity measurements with the reference velocity at different stationary angles of attack. According to the methodology and calibration process, the calibration function already obtained from the static tests, Eq. (6.1), is used to correct the UA measurements in dynamic cases. The calibration coefficients, $A$ and $B$ tabulated in Table 8 , are obtained for all the instantaneous azimuthal angles, $\theta(t)$, in rotational motion and is used to correct the velocity measurements in the form of $U_{c}(t)=A(t) U_{s}(t)+B(t)$.

Figure 6.21 (a) illustrates the non-dimensional velocity measured by the UA, $U_{s}(t) / U_{\infty}$., and the reference velocity, $U_{r}(t)$, for a typical dynamic test. Reference velocity is the ideal response that is expected to be measured by the spinning UA at each instantaneous azimuthal angles, $U_{r}(t)=U_{\infty} \cos \theta(t)$. Remarkable discrepancy in the measurements can be observed when the anemometer is crossing the azimuthal angles of $0^{\circ}, 180^{\circ}$ and $360^{\circ}$ where the transducer head distortions are at their maximum level and considerably affect the measurement path. 
Figure 6.21 (b) shows the non-dimensional calibrated wind speed measurements, $U_{c}(t) / U_{\infty}$, and the reference velocity, $U_{r}(t)$, for the same presented typical test. This figure shows that the calibrated measurements are in a good agreement with the reference velocity.

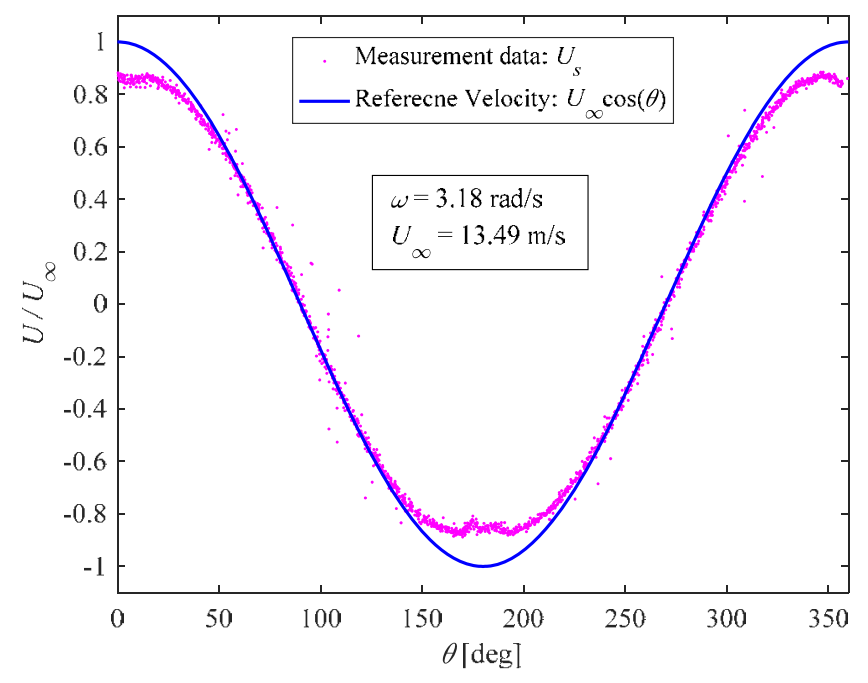

(a)

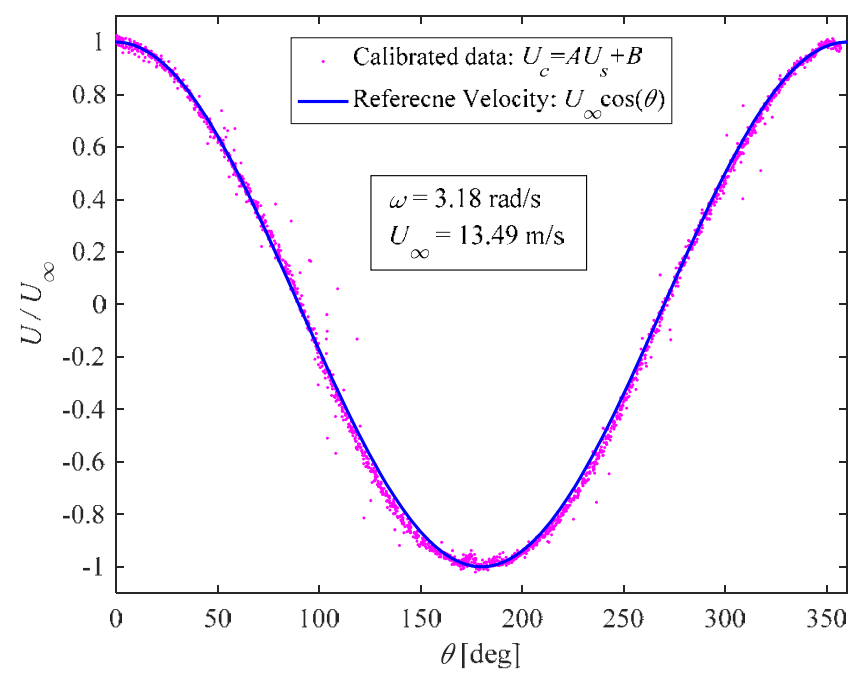

(b)

Fig. 6.21: Comparison of ultrasonic velocity measurements with reference velocities

(a), and calibrated velocity measurements with reference velocities (b).

To determine the accuracy of the calibration process, the coefficient of determination, $R^{2}$, is calculated to indicate the performance of the calibration function, $U_{c}(t)$, in comparison with the reference velocity. Shown in Fig. 6.22 is the $R^{2}$ for fitting the calibrated measurements and reference velocity, for various wind speeds and UA angular velocities. Even though for high rotational velocities a slight reduction is observed in $R^{2}$, an average value of $R^{2} \approx 0.997$ is achieved throughout the test conditions and approves the high accuracy of the calibration process. 

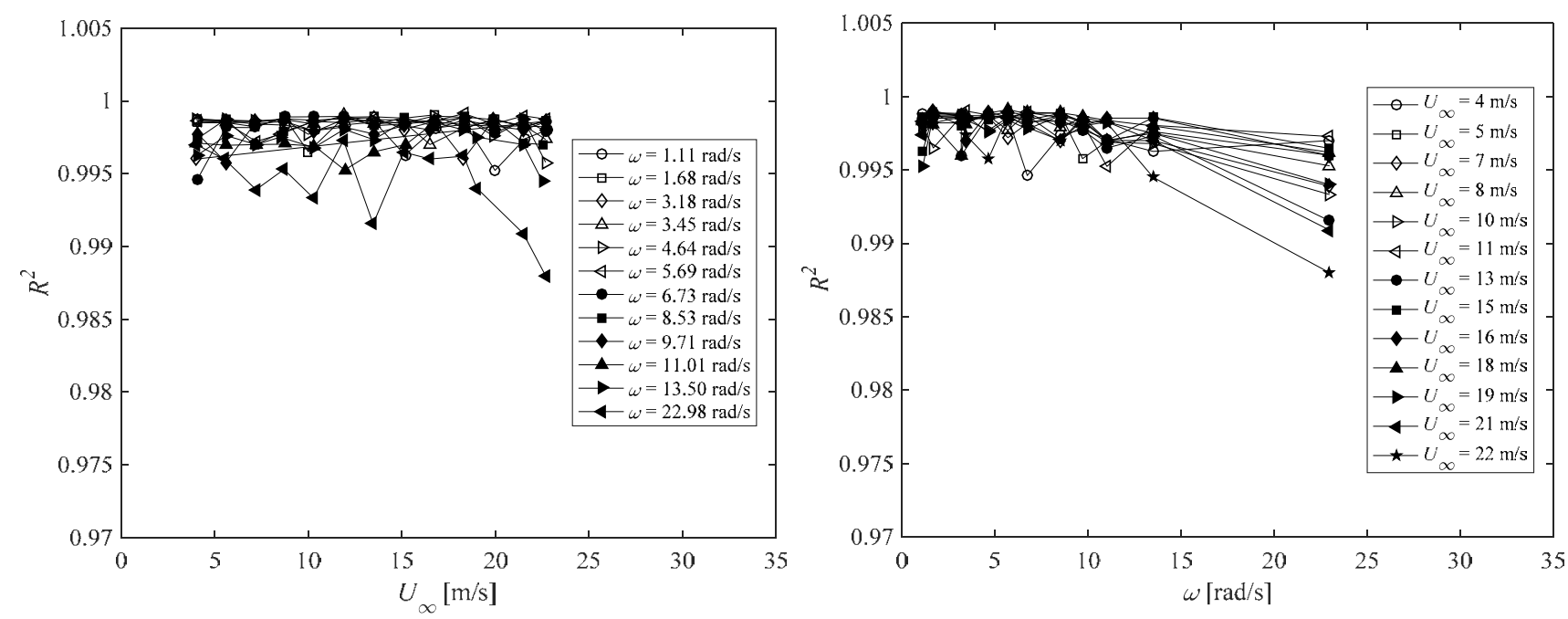

Fig. 6.22: Regression coefficient, $R^{2}$, for the fitting of the calibrated measurements, $U_{c}(t)$ with the reference velocities, $U_{r}(t)$ as a function of the rotating velocities (Left) and of the wind-tunnel flow velocities (Right).

\subsubsection{Phase angle in dynamic tests}

In this section, for validating the obtained calibration model in Eq. (6.8), some of the dynamic tests tabulated in Table 12 are chosen in order to see how the proposed calibration function corrects the UA velocity measurements during the rotation.

Table 12: Wind speeds and UA angular speeds of some typical dynamic cases.

\begin{tabular}{|c|c|}
\hline Wind speed, $U_{\infty}[\mathrm{m} / \mathrm{s}]$ & Angular speed, $\omega[\mathrm{rad} / \mathrm{s}]$ \\
\hline 5.61 & 1.68 \\
\hline 8.73 & 3.18 \\
\hline 13.50 & 5.69 \\
\hline 16.73 & 6.73 \\
\hline 22.73 & 11.01 \\
\hline
\end{tabular}

Fig. 6.23 (left) illustrates the velocity measured by the spinning $U A, U_{s}(t)$, for a typical test with $U_{\infty}=13.50 \mathrm{~m} / \mathrm{s}$ and $\omega=3.18 \mathrm{rad} / \mathrm{s}$. It is observed that in the shadow regions, as explained earlier, the velocity measured by the UA is differed with the reference velocity. This discrepancy is occurred when the anemometer is crossing the azimuthal angles of 
$0^{\circ}, 180^{\circ}$ and $360^{\circ}$ where the signal path is aligned to the wind flow direction and the maximum sensor head distortions are shed into the measurement path.

In each test, the phase angle, $\phi(t)$, for each instantaneous azimuthal angles, $\theta(t)$, can be calculated by Eq. (6.6) and is used to calibrate the velocity measurements in the form of $U_{c}(t)=U s(t) /[\cos (\phi(t)-\tan (\theta(t)) \times \sin (\phi(t))]$.

The calibrated velocity measurements by UA and the reference velocity for the same presented typical test are illustrated in Fig. 6.23 (right). Recall that the reference velocity is the ideal response that is expected to be measured by the spinning UA at each instantaneous azimuthal angles, $U_{r}(t)=U_{\infty} \cos \theta(t)$. From the Fig. 6.23 (right), it is seen that the calibrated values are in a very good agreement with the reference velocity.
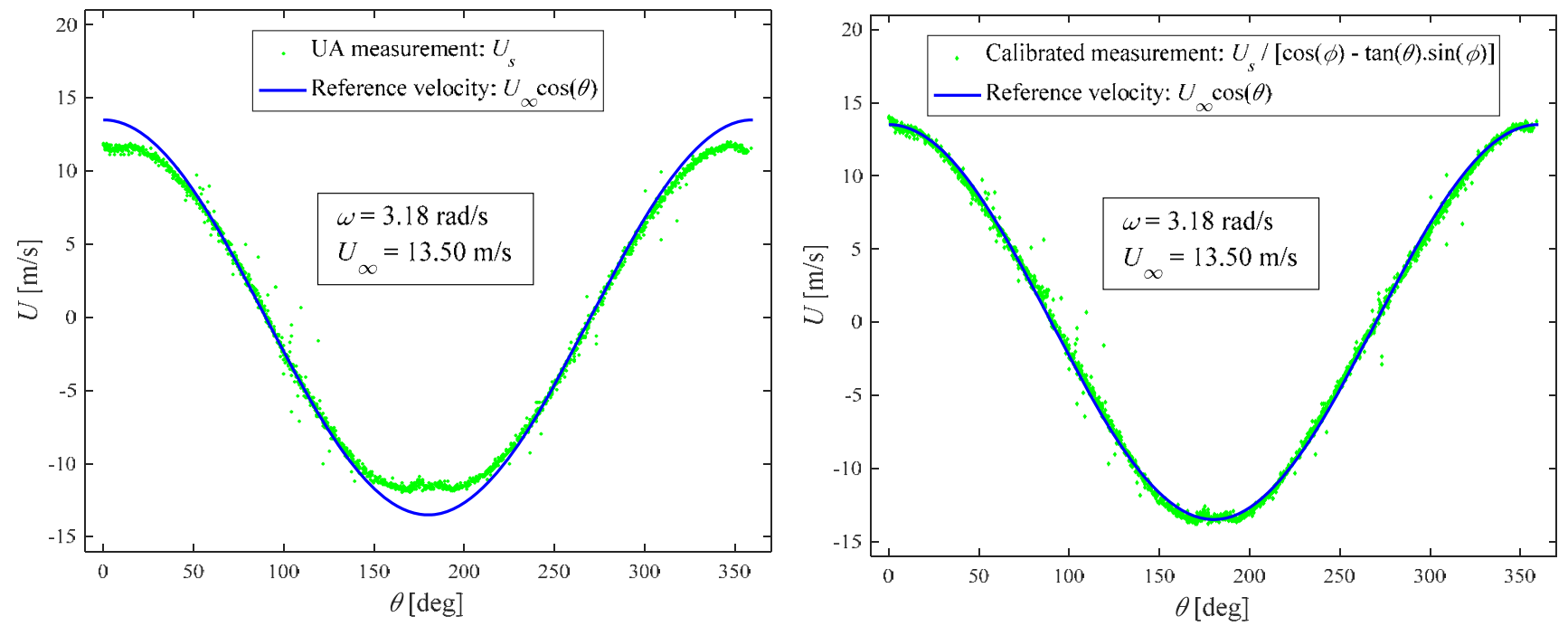

Fig. 6.23: UA measurements and reference velocity (left), and calibrated velocity measurements and reference velocity (right) for a typical spinning test.

To determine the accuracy of the calibration model, the Root Mean Square Error (RMSE), is calculated to indicate the performance of the calibration function, $U_{c}(t)$, to correct the UA measurements, $U_{s}(t)$.

To construct the RMSE, it is needed to determine the residuals. Residuals are the difference between the reference values and the corrected values. Here, they are denoted by $\widehat{y}_{l}-y_{i}$, where $\widehat{y}_{l}$ is the corrected values by the calibration model $\left(U_{c}\right)$, and $y_{i}$ is the reference values $\left(U_{r}\right)$. Residuals can be positive or negative as the corrected value under or over estimates the reference value. Squaring the residuals, averaging the squares, and taking the square root gives the RMSE. Then the RMSE is calculated by Eq. (6.11): 


$$
R M S E=\sqrt{\frac{\sum_{i=1}^{n}\left(\widehat{y}_{l}-y_{i}\right)^{2}}{n}}
$$

Shown in Fig. 6.24 is the RMSE for fitting the calibrated model and reference model, for various wind speeds and UA rotational velocities. It is seen that the UA rotational speeds do not influence on the accuracy of the calibration model. Although, the RMSE is increased slightly by the wind speed, its values are varied between 0 to 1 which approves the data is concentrated well around the calibration model. The small values for RMSE indicates the high accuracy of the calibration procedure and the obtained model for correcting the wind speed measurements with the spinning ultrasonic wind sensor in both static and spinning cases.
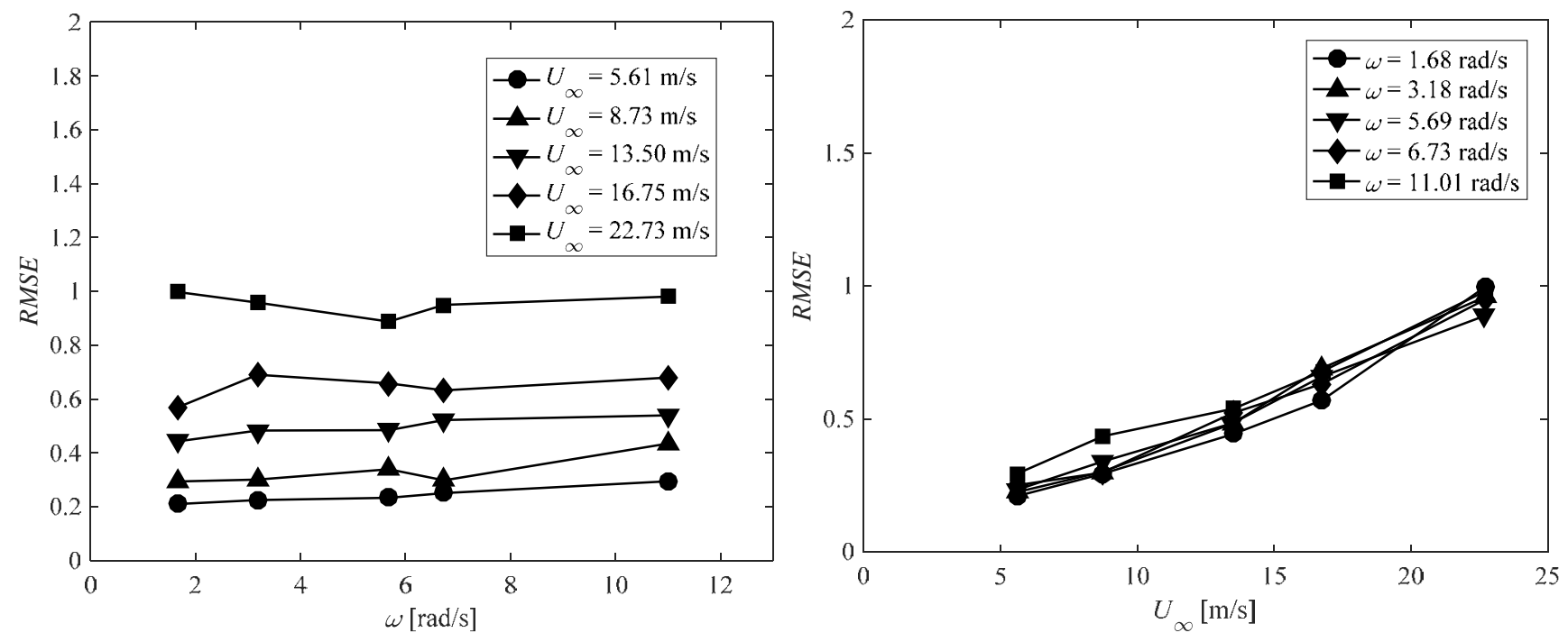

Fig. 6.24: RMSE for variation of the wind speed and UA angular speed. 


\subsection{Outlines of the chapter}

A modified single-axis ultrasonic anemometer was used to measure the wind speed in a subsonic wind tunnel. The experiments were performed for both static and dynamic oscillatory cases. In static tests, the velocity measured by the ultrasonic anemometer in different stationary azimuthal angles of signal path with respect to wind direction has been calibrated with wind-tunnel reference wind speed measured by two pitot-tubes.

The calibration coefficients obtained in the static tests were then extended to the dynamic cases where the UA was rotating with different angular velocities at various wind speeds. The calibrated measurements showed a good agreement with the reference velocity which is defined as the projection of the wind-tunnel wind speed vector onto the signal path orientation. An average of $R^{2}=99.7 \%$ was achieved so that the uncertainty in the dynamic tests due to transducer shadow effect is almost $0.3 \%$.

It is concluded that the measurements are affected mostly with the transducers shadow effect at each instantaneous azimuthal angle, and the shifting in acoustic pulse trajectory does not affect the UA wind speed measurements in rotational motion. This is actually confirming that the static shadow correction can be used for correcting the same anemometer's measurements in the spinning cases.

Besides, a phase angle as a function of the orientation of UA signal path is obtained from the static tests which is due to the transducer shadow effect. The phase angle can be used to calibrate the wind speed measured by UA in both static and dynamic cases. The results showed that the proposed calibration function for the transducer shadow correction works satisfactorily for the spinning UA and can correct its velocity measurements at each instantaneous azimuthal angles.

Therefore, the calibration function for a fixed single-axis UA in different stationary angles of signal path with wind direction at a plane can be used to the same anemometer in rotational motion at that plane. This procedure works for any other single axis UA as well. It must first be calibrated in various stationary yaw or pitch angles to determine the calibration constants. It has been shown that the same constants can be used to construct a correction model in the spinning case. 


\section{Chapter 7}

\section{Response Surface Methodology (RSM)}

The statistical method using response surface methodology (RSM) has become a popular tool to determine the influences of individual factors and their interactions on a dependent variable $[69,70]$. In this method, a response surface can be constructed based on experimental data so that the dependent variable is expressed in the form of a polynomial, usually a quadratic type, in terms of the independent variables. Other than providing an analytic equation between the dependent or response variable and the independent ones, RSM is a technique for designing experiments and evaluating the effects of several factors. RSM describes the way a particular response variable is influenced by a set of independent inputs [71].

In this thesis, extensive wind tunnel tests were performed on a spinning single-axis UA and the response surface method was exploited to construct a theoretical model from the experimental results to relate the velocity measured by the UA to the free stream velocity and the rotation parameters. With this expression, the output measurements of the anemometer can easily be calibrated to give the oncoming wind speed at each instantaneous azimuthal angle.

Besides, as it was discussed in the section 6.3.2, the present experimental results revealed a phase shift in the angular positions relative to wind speed measured by the spinning UA. This shifting phenomenon is occurred because of the time delay between 
UA and angular sensor measurements during the rotational motion. In this chapter, based on response surface methodology, a mathematical model is proposed to calculate the phase shifting phenomenon observed in spinning ultrasonic wind speed measurements, and correct the velocity read by the UA for this phase shift. In a broader sense, this provides a practical means to account for the phase shift in ultrasonic wind measuring technology and calibrate the yaw misalignment in spinner anemometry.

The Response Surface Methodology (RSM) encompasses a collection of mathematical and statistical techniques to model, analyze and optimize the problems in which a certain dependent variable is influenced by some independent variables [72, 75]. There exist various designs and models in a RSM structure to work with continuous treatments and are used to describe the system response to the independent input variables. It is also used in other applications such as the optimization problems. The main task in RSM is to model the changes in response variable for a given set of independent parameters [74].

For $q$ independent variables, $x_{1}, x_{2}, \ldots, x_{q}$, the dependent or response parameter denoted by $y$ can be expressed as an unknown function of the independent variables, i.e. $y=f\left(x_{1}, x_{2}, \ldots, x_{q}\right)$. Usually, in RSM problems, the function $f$ is unknown and to get a good approximation for $f$, a low-order, e.g. a first or second order polynomial in a narrow range of the independent variables should be exploited, depending on the complexity of the problem.

The approximated function $f$ is a first-order model when the response is a linear function of the independent variables. A first-order model with $N$ experimental runs encountering $q$ design variables and a single response $y$ can be expressed in the form of Eq. (7.1):

$$
y_{i}=\beta_{0}+\beta_{1} x_{i 1}+\beta_{2} x_{i 2}+\cdots \cdots+\beta_{q} x_{i q}+\varepsilon_{i} \quad(i=1,2, \ldots \ldots, N)
$$

The response $y$ is a function of the design variables $x_{1}, x_{2}, \ldots, x_{q}$, denoted as $f$, plus the experimental error. A first-order model is a multiple-regression model and the $\beta_{j}$ 's are regression coefficients. First-order model is used to describe the flat response surfaces.

Depending on complexity and nature of the problem under consideration, a first, second or higher order polynomial or any other appropriate mathematical function should be considered to describe $f$. This model is assumed to be an adequate approximation of the actual response in a small region of $x$ 's [Montgomery (2005)]. A second order- two variables model for example, can be considered in the form of Eq. (7.2): 


$$
y=\beta_{0}+\beta_{1} x_{1}+\beta_{2} x_{2}+\beta_{11} x_{11}^{2}+\beta_{22} x_{22}^{2}+\beta_{12} x_{1} x_{2}+\varepsilon
$$

The unknown constants $\beta_{\mathrm{i}}$ in Eq. (7.1) or $\beta_{\mathrm{ij}}$ in Eq. (7.2) are determined by the Least Square method based on the $N$ sets of experiments in which, the response variable has been measured for a certain set of the independent variables.

In General, most of RSM problems use either first or second order model or a combination of both [75]. The unknown constants $\beta_{\mathrm{i}}$ in Eq. (7.1) or $\beta_{\mathrm{ij}}$ in Eq. (7.2) are determined by the Least Square method based on the $N$ sets of experiments in which, the response variable has been measured for a certain set of the independent variables.

The classic RSM was first introduced by Box and Wilson [76]. They proposed a first degree polynomial model to approximate the response variable. This model despite being an approximation is easy to use even for fairly complicated applications such as highly nonlinear or time dependent problems in which, little is known about the process.

In most of the modern practical problems, the existence of a maximum or a minimum or a saddle point is of great importance, especially in engineering applications. This has highlighted the role of RSM in such problems. Also, in recent years more emphasis has been placed by the wind-tunnel testing to simulate the flow behavior on different bodies to find the regions where there is an improvement in response instead of finding the optimum response. As a result, application and development of RSM will continue to be used in many areas in the future.

In the past two decades, many attempts have been made to exploit the philosophy of the design of experiments and the response surface method in the wind-tunnel tests. Extensive research program has been commenced in NASA wind-tunnels since 1990 to reduce the time and cost of the experiments by reducing the number of tests [77] and further, to predict the aerodynamic and performance variables using the modest amount of runs by taking the advantages of RSM [78, 79].

In a response surface problem, it is desired to obtain an accurate model with minimum number of polynomial coefficients. The traditional process to find the proper polynomial terms to be included in the model for a given set of data is usually performed by trial-anderror, or by just including all polynomial terms that could possibly be identified from the experiment [74]. 


\subsection{RSM to calibrate UA for Yaw Misalignment}

The present experimental results revealed a phase shift in angular positions relative to wind speed measured by the spinning UA. This shifting phenomenon is occurred because of the time delay between UA and angular sensor measurements along with DopplerEffect during the rotational motion appearing as a yaw misalignment in each test.

The phase shift, once expressed in terms of the convenient independent variables, can easily be modeled to correct the UA output signal. Here, the response surface method has been employed to construct a regression model from the experimental data.

The independent parameters responsible for the phase shift are identified to be the freestream wind velocity, $U_{\infty}$, the rotational speed, $\omega$, the time delay between two consecutive acoustic pulses, $z$, an azimuthal angle, $\theta_{o}$, which is defined earlier as $\theta_{e}$ where UA measures the first zero wind speed, and of course the UA geometry, i.e. its acoustic path length, $L$. However, only one UA has been examined in the present experiments with a fixed geometry. Re-grouping and combining the independent variables, the phase shift, $\Delta \theta$, is considered to be a function of three independent variables as given below:

The reduced frequency, $k$, which is the ratio of the UA rotational velocity to the wind speed. Alternatively, it can be thought as the ratio of the time required for a fluid particle to travel between the UA heads to that required for the UA to rotate 360 degrees in a complete cycle.

Second is the time delay parameter that normally exist between pulses firings. Cuerva et al. [80] suggested that both the acoustic path length, $L$, and the speed of sound, $c$, are responsible for the time delay between two consecutive pulses firings. He expressed the time delay in terms of these variables as $z=L /\left[c \times\left(1-U_{\infty} / c\right)\right]$, where $U_{\infty}$ is the mean freestream wind speed and $L$ and $c$ have already been defined as the length of the acoustic path, and the speed of sound.

A tertiary parameter, playing a vital role in phase shift, has been inspired from the corrections proposed in previous researches [2, 53, 55] for shadow effect corrections, which contain an exponential sin-function $\left(\exp \left(\sin ^{2} \theta\right)\right)$. Note that the rotation of the UA heads has a sinusoidal behavior and the corresponding rotational speed would be the time derivative of this sine function, i.e. in a cosine form. The expected velocity read by the UA is also equal to $U_{\infty} \cos (\theta)$. Thus, it is believed that both the sine and cosine functions of the azimuthal angle, $\theta_{o}$, are responsible for this time shift. Retaining the exponential function, the authors suggest that the time shift can be deemed to be partially a function of $\exp \left(\tan \theta_{o}\right)$ as a single combinational independent variable. 
Figure 7.1 (a, b, and c) illustrates the surface plot of the response variable, $\Delta \theta$, for various reduced frequencies, time delay and $\exp \left(\tan \theta_{0}\right)$ parameters. The non-smooth and wavy surface is observed in three plots. This implies that high interactions exist between the independent variables, $k, z$ and $\exp \left(\tan \theta_{0}\right)$.

A response surface model for phase shift, based on foregoing arguments, can be proposed in the form of:

$$
\Delta \theta=\beta_{1} k+\beta_{2} z+\beta_{3} e^{\tan \left(\theta_{o}\right)}
$$

Using a multiple regression on the experimental data, the unknown parameters are determined:
$\beta_{1}=-0.397$
$\beta_{2}=421.2$
$\beta_{3}=1.4276$ 
(a):

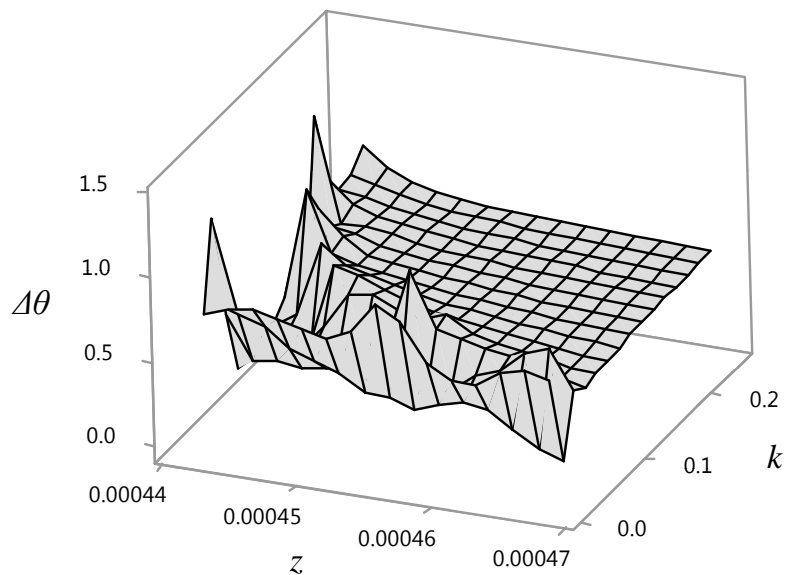

(b):

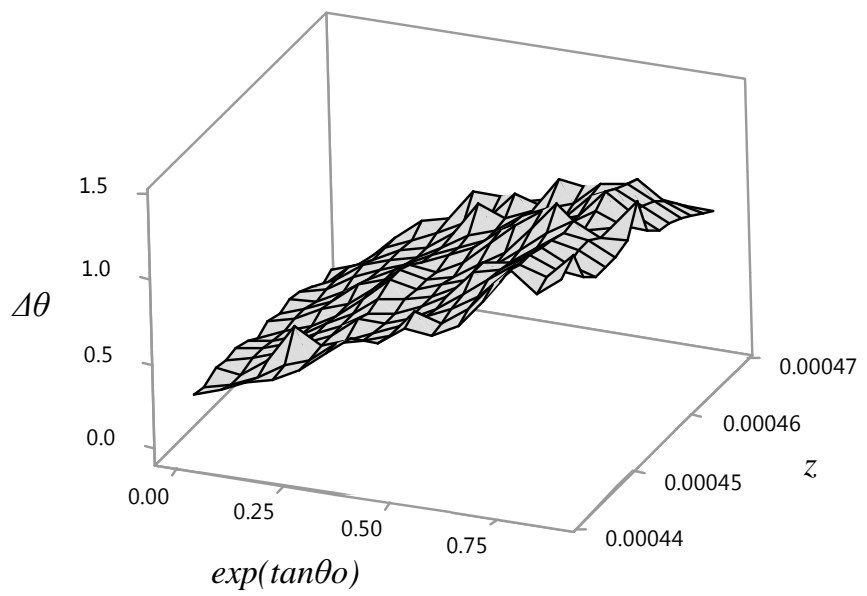

(c):

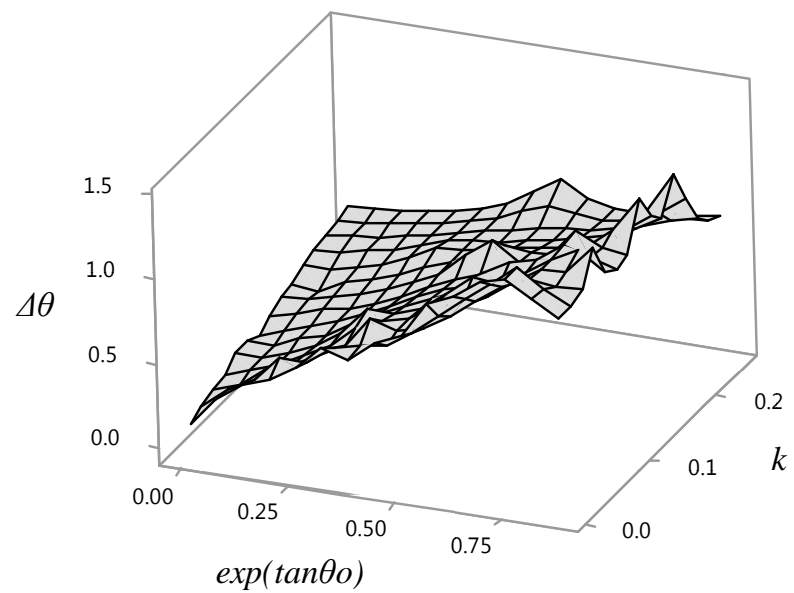

Fig. 7.1: Response surface of the phase shift based on the independent variables. 
Eq. (7.3) gives an estimation of the phase shift for given values of $z, k$ and $\theta_{0}$. Basically, the error term or residual is defined as the difference between an observed value and the corresponding fitted value predicted by the response surface. In the present work, the residual is expressed as $e_{i}=\Delta \theta_{i}-\Delta \hat{\theta}_{i}$ where $\Delta \theta_{i}$ is the phase shift measured from the experiment and $\Delta \hat{\theta}_{i}$ is the one predicted by Eq. (7.3). This error must be normally and independently distributed with zero mean. The normal probability plot is a measure of how normally the residuals are distributed [72]. Shown in Fig. 7.2 is the normal probability distribution for Eq. (7.3). A linear distribution can be observed approving the accuracy of the model in comparison to the actual data obtained in the wind-tunnel.

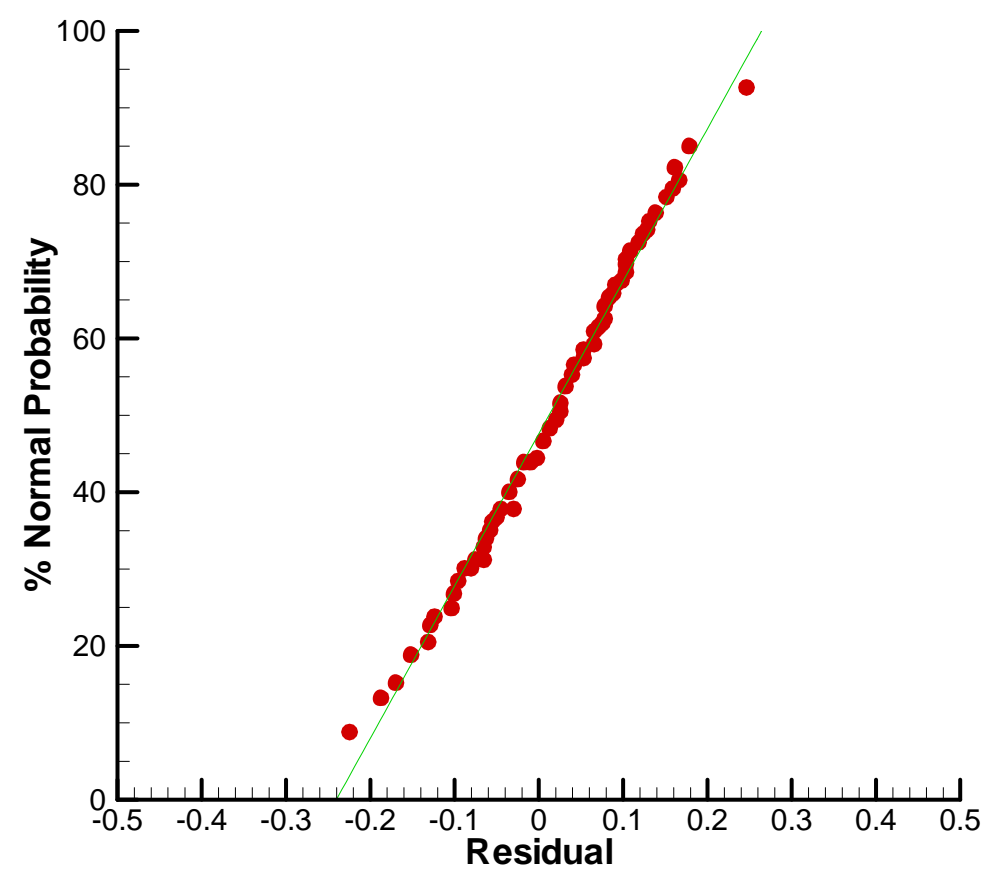

Fig. 7.2: Normal probability distribution of Eq. (7.3).

The estimation residuals of the fitted values have been illustrated in Fig. 7.3. According to this figure, the residuals, which are the difference between the measured value and the corresponding fitted value predicted by the response surface in Eq. (7.3), are symmetrically located around zero mean. This confirms that the estimation errors encountered in the regression model are not of systematic type and can really be considered as noises in the model. 


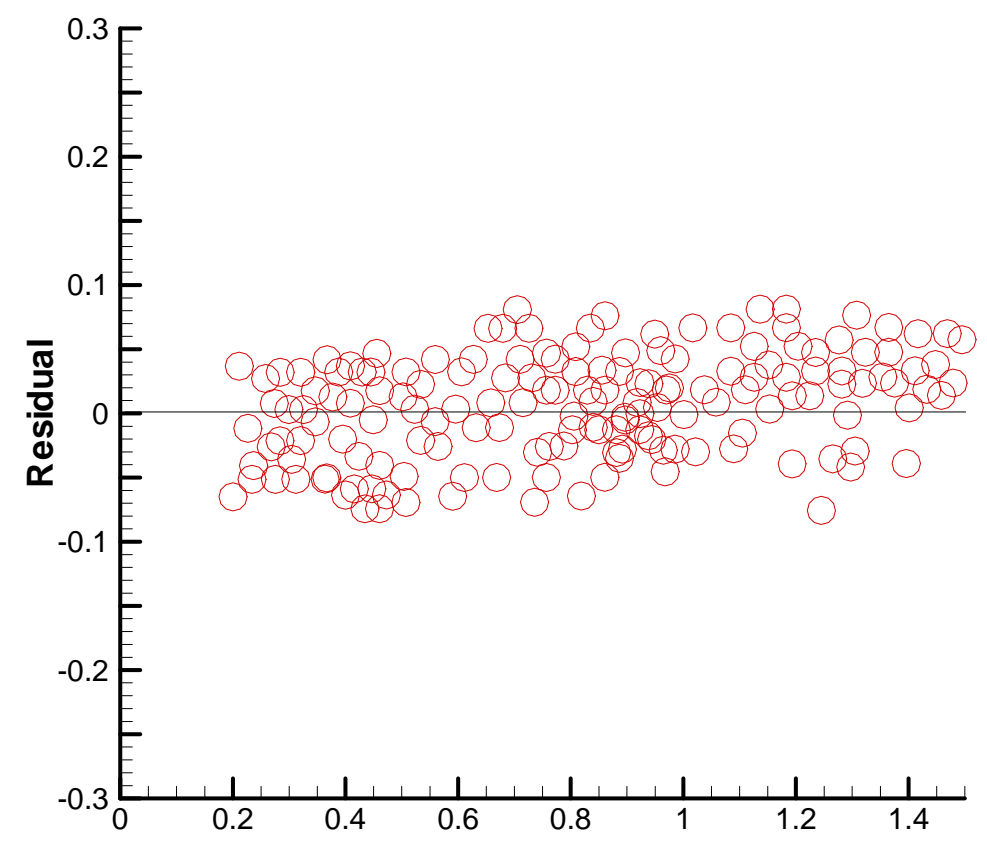

Fitted Value

Fig. 7.3: Distribution of the residuals in Eq. (7.3).

To check the model adequacy, all of the terms added to the response surface model of Eq. (7.3) must be verified to be the major contributors to the response variable. The hypothesis test to determine the p-value [73] can approve it. For each term in the regression, the null hypothesis implies that the term under consideration does not have any significant effect on the response variable. If the p-value from the Anderson-Darling test [81] for that term is less than a certain significance level, (usually 0.05), there would be enough evidence to reject the null hypothesis. In this case, the term for which, the pvalue has been determined, must be thought to be effective on the response.

Variance inflation factors (VIF), is also another parameter to check the degree of correlation between the independent variables [82]. It is simply calculated from Eq. (7.3):

$$
V I F=\left(1-R^{2}\right)^{-1}
$$

where $R^{2}$ is the coefficient of determination to be discussed in the next paragraphs. Actually, VIF shows the variance inflation of the estimated regression coefficients in comparison to the case when there is not a linear dependence between the independent variables. In a regression analysis it is used to determine how much multi-collinearity, i.e. correlation between the independent variables exists [83]. 
If VIF $=1$, the predictors are not correlated. For the values of VIF between 1 and 5 , the predictors are moderately correlated and VIF larger or equal to 5 indicates that the predictors are highly correlated.

For a significance level of 0.05 , the p-value and VIF for each term have been tabulated in Table 13. On this basis, within 95\% confidence level, all of the terms in Eq. (7.3) can be considered to be highly correlated and have significant effect on the response variable, i.e. the phase shift.

Table 13: P-values and VIF for each term in the regression mode.

\begin{tabular}{ccc}
\hline Term & P-value & VIF \\
\hline $\mathbf{k}$ & 0.008 & 2.13 \\
$\mathbf{z}$ & 0.000 & 2.55 \\
$\mathbf{e}^{(\tan \theta \boldsymbol{o})}$ & 0.000 & 1.90 \\
\hline
\end{tabular}

The coefficient of determination in a response surface model, $R^{2}$, which is between 0 and 1 , indicates how well the estimated model fit the data. Theoretically, the closer be $R^{2}$ to the unity, the better would be the estimation of regression equation. However, in many practical applications of a response surface model, the large value of $R^{2}$ cannot necessarily approve the regression quality. In most cases, $R^{2}$ tends to increase when the response model structure changes or a new variable, regardless of its significance, is introduced in the regression model.

As an alternative, adjusted regression, $R^{2} a d j$ is frequently used instead. If unnecessary variables are added to the response model, the value of $R^{2}$ adj often decrease. Basically, $R^{2}$ indicates how much of the observed variability in the data was accounted for, by the model while the $R^{2}$ adj modifies $R^{2}$ by taking into account the number of covariates or predictors in the model.

In a set of data, the total sum of squares, SST, the regression sum of squares, SSR, and the error sum of squares, SSE, are the key statistical parameters to describe the performance of a response variable prediction. These parameters show the difference between the observed response variable, $y_{i}$, and that predicted by the regression model, $\widehat{y}_{l}$, about a mean value, $\bar{y}[84]$ : 


$$
S S T O=\sum_{i=1}^{n_{S}}\left(y_{i}-\bar{y}\right)^{2} . S S R=\sum_{i=1}^{n_{S}}\left(\widehat{y}_{l}-\bar{y}\right)^{2} . S S E=\sum_{i=1}^{n_{s}} \varepsilon_{i}
$$

$R^{2}$ is defined as:

$$
R^{2}=\frac{S S R}{S S T O}=1-\frac{S S E}{S S T O}
$$

And the $\operatorname{Radj}^{2}$ is:

$$
R_{a d j}^{2}=1-\frac{n-1}{n-p}\left(1-R^{2}\right)
$$

where $n$ is the number of the observations or measurements and $p$ is the number of predictors (terms) in the model, excluding the constant term [85]. For the response surface model proposed in Eq. (7.3) the $\operatorname{Radj}^{2}$ has been measured to be $99.03 \%$ which approves the accuracy and performance of this model to predict the phase shift in the velocity read by the UA.

The response surface model for phase shift, constructed from the experimental data, was used to correct the velocity read by the UA. Fig. 7.4 shows the corrected measurements for phase shifting for some typical tests as their raw data were presented in Fig. 6.18. The velocity measured by the UA, once taken into account the correction for yaw misalignment during rotation, shows a remarkable agreement with the expected values which are $U_{\infty} \cos (\theta)$. The RSM satisfactorily works for various free stream wind speeds and UA rotational speeds. However, some discrepancies are observed at $\theta=0$ and 180 degrees which is due to transducer shadow effect, discussed earlier.

Even though the RSM model proposed in this paper was constructed from windtunnel tests for a specific UA, the authors believe that the approach works as well for any other UA in different conditions and different geometries, with the same model structure and evidently with different regression coefficients, $\beta$ i. 


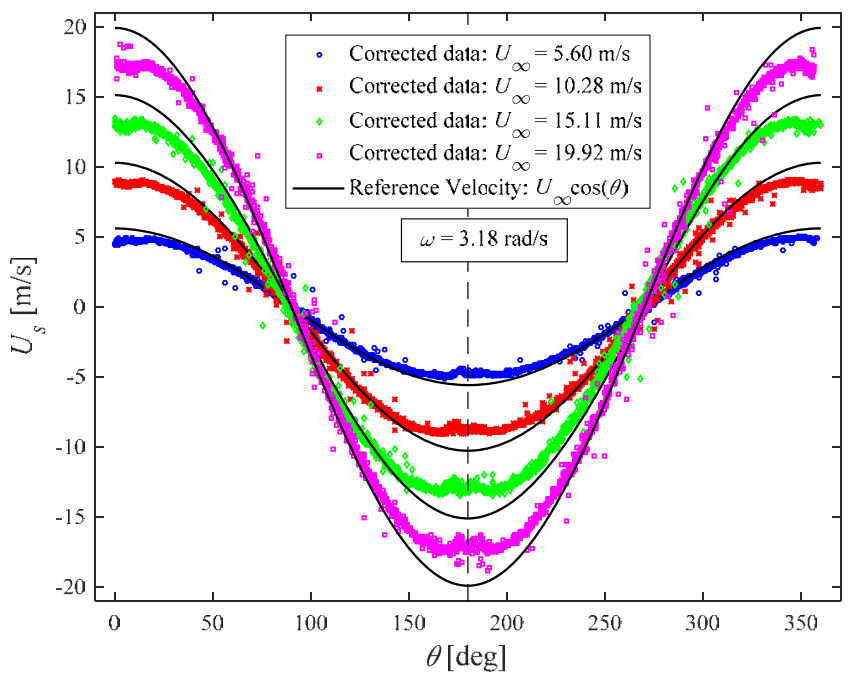

(a)

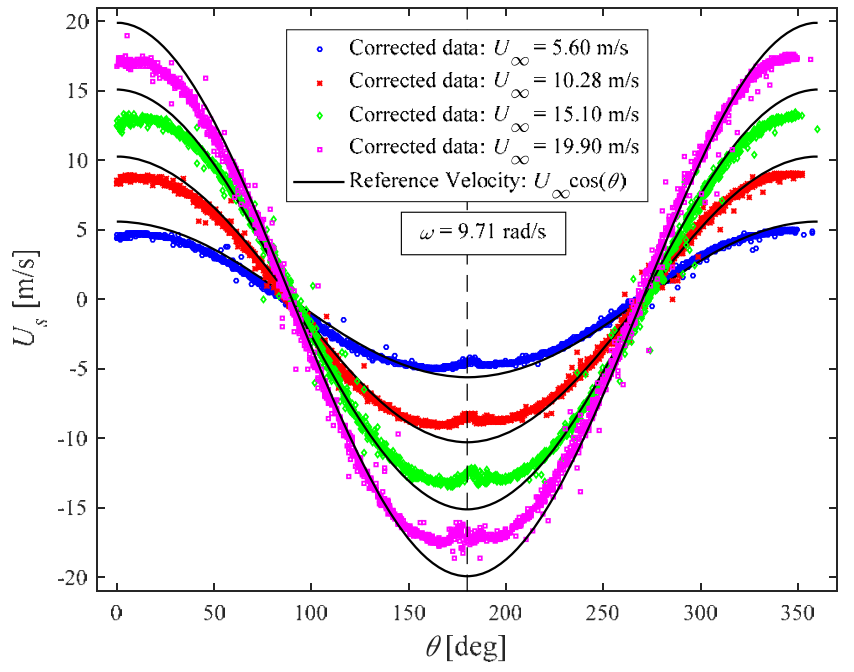

(b)

Fig. 7.4: Corrected data for yaw misalignment of the velocities measured by UA for various incoming wind speeds at (a) low rotational speed and (b) high rotational speed. 


\subsection{RSM to calibrate UA for wind speed measurements}

A sonic anemometer was rotating inside the wind-tunnel test chamber. Wind speed measured by the UA, instantaneous angular position of the UA, and wind speed measured by the pitot-tube have been recorded at the same time during the experiments. Several replications have been performed during the test to reduce the uncertainty of the data and check the performance of the theoretical model extracted from the present experiments.

In the present experiments, the velocity measured by the sonic anemometer, $U_{s}$, is the response and is found to be a function of free stream wind speed, $U_{\infty}$, rotational speed, $\omega$, instantaneous azimuthal angle, $\theta$, and of course, the UA geometry, i.e. its acoustic path length, $L$ [53]. Fig. 7.5 (a, and b) illustrates the response surface plot of the dependent (response) variable, $U_{s}$, as a function of the independent variables: UA angular positions $(\theta)$, UA angular velocity $(\omega)$, and free stream wind speed $\left(U_{\infty}\right)$. The non-smooth and wavy surface in this figure suggests that the response variable is not a function of the linear combination of each of the independent variables and the products of them are also important. Thus, high interactions between the independent variables, $U_{\infty}, \theta$ and $\omega$ should be considered. For this reason, a second order response surface has been established. In this model, the products of the independent variables are included to the regression as well as the linear combination of them.

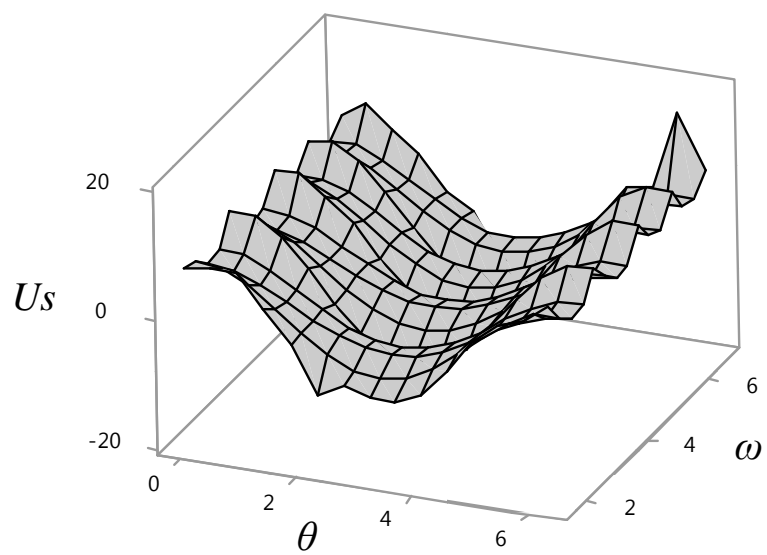

(a)

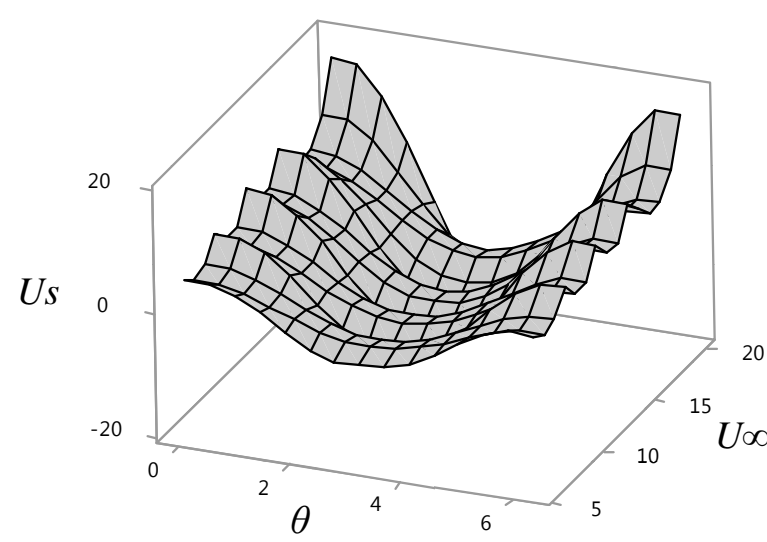

(b)

Fig. 7.5: Response surface plot of the UA indicated velocity, $U_{s}$, as a function of the independent factors: azimuthal angles $\theta$, angular velocities $\omega$, wind speeds $U_{\infty}$.

Note that the UA measurements in general is a function of the radial component of the wind speed, i.e. $U_{\infty} \cos \theta$, while in the shadow regions, the tangential component, $U_{\infty} \sin \theta$, 
seems to play a major role in the velocity measured by the UA. Since both the UA angular velocity and the tangential component of wind speed are dominant parameters in the shadow region, the second order model is proposed in the form of Eq. (7.8) to predict the velocity measured by the UA. Re-grouping and combining the independent variables, the angular velocity has been expressed in terms of the reduced frequency, $k=\omega L / 2 U_{\infty}$.

$$
\begin{gathered}
U_{s}=\beta_{0}+\beta_{1} U_{\infty} \cos \theta+\beta_{2} U_{\infty} \sin \theta+\beta_{3} k+\beta_{12} U_{\infty}^{2} \cos \theta \sin \theta \\
+\beta_{13} k U_{\infty} \cos \theta+\beta_{23} k U_{\infty} \sin \theta
\end{gathered}
$$

Using a multiple regression on the experimental data, the unknown constants $\beta_{i j}$ are determined:

$$
\begin{array}{llll}
\beta_{0}=0.0724 & \beta_{1}=0.91750 & \beta_{2}=-0.01058 & \beta_{3}=-1.567 \\
\beta_{12}=0.000086 & \beta_{13}=-0.099 & \beta_{23}=-0.106 &
\end{array}
$$

The prediction error is defined as $e_{i}=y_{i}-\hat{y}_{i}$, where $y_{i}$ is measured from the experiment and $\hat{y}_{i}$ is predicted by the RSM. In an ideal prediction problem, the mean value of $e_{i}$ is zero.

The normal probability plot shows the distribution of the estimated residuals [86]. Residual is defined as the difference between an observed value and the corresponding fitted value predicted by the response surface model. The estimation residuals have been shown in Fig. 7.6 and illustrates that the response model considered in Eq. (7.8) are not linearly distributed. This shows a poorly formulated response model because the residuals have not fallen on a straight line in the normal probability plot. It can be inferred that the regression terms in Eq. (7.8) could not properly model the response. Furthermore, the residuals shown in this figure are not symmetrically located around zero, violating a key assumption of linear regression. 


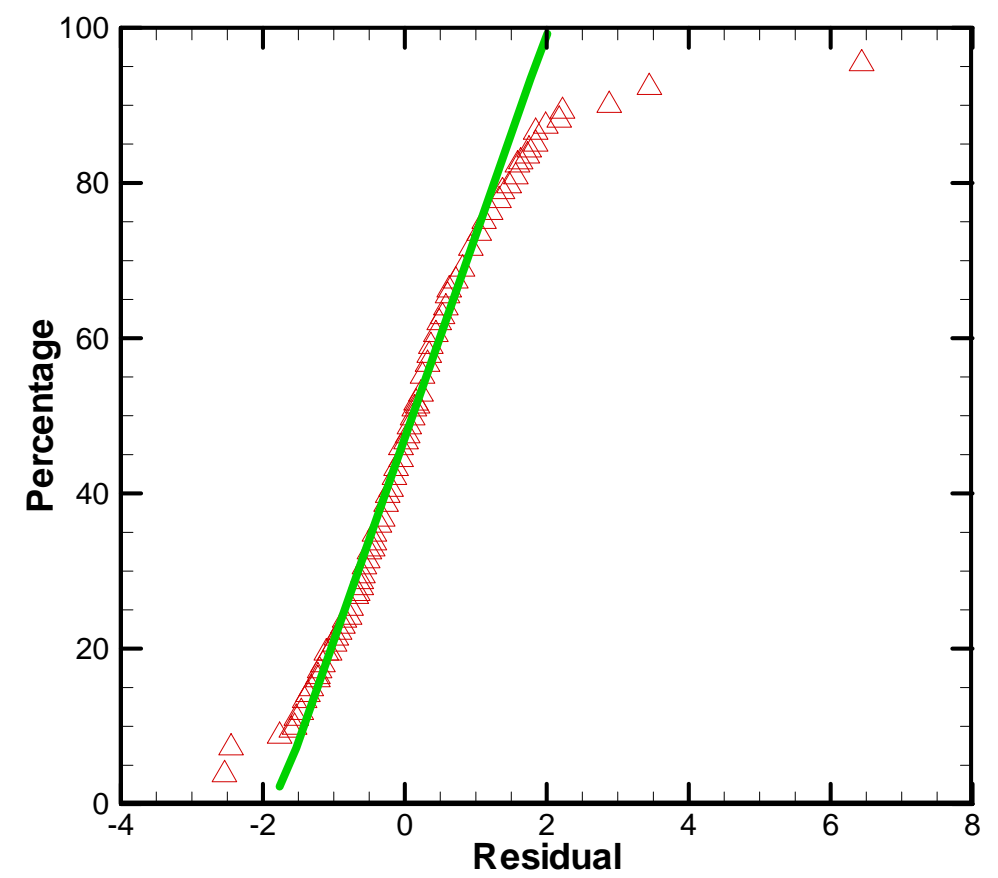

Fig. 7.6: The normal probability of the residuals in Eq. (7.8).

To make sure that all of the terms added to the response surface model of Eq. (7.8) were the major contributors to the response variable, the hypothesis test is conducted to determine the p-value [73]. For each term in the regression, the null hypothesis implies that the term under consideration does not have any significant effect on the response variable. From the Anderson-Darling test [81], if the p-value for that term is greater than a certain pre-defined value, known as significance level and is usually set to 0.05 , the null hypothesis is accepted. This means that the term under consideration does not have any significant on velocity measured by the UA.

On the other hand, if the p-value is less than the significance level, there would be enough evidence to reject the null hypothesis. In this case, the term must be considered to be effective on the UA measurement. The p-value for each term in Eq. (7.8) has been tabulated in Table 14 for a significance level of 0.05 .

Table 14: P-values for the regression terms in Eq. (7.8).

\begin{tabular}{|l|l|l|l|l|l|l|l|}
\hline Term & Constant & $U_{\infty} \cos \theta$ & $U_{\infty} \sin \theta$ & $k$ & $U_{\infty}{ }^{2} \cos \theta \sin \theta$ & $k U_{\infty} \cos \theta$ & $k U_{\infty} \sin \theta$ \\
\hline p-value & 0.001 & 0.000 & 0.000 & 0.017 & 0.586 & 0.344 & 0.310 \\
\hline
\end{tabular}

On this basis, within $95 \%$ confidence level, the $5^{\text {th }}, 6^{\text {th }}$ and $7^{\text {th }}$ terms in Eq. (7.8) can be considered to have no significant effect on the velocity measured by the UA and can 
be removed from the response model. The modified response model can thus be proposed in the form of Eq. (7.9):

$$
U_{s}=U_{s}=\beta_{0}+\beta_{1} U_{\infty} \cos \theta+\beta_{2} U_{\infty} \sin \theta+\beta_{3} k
$$

With the following coefficients:

$$
\beta_{0}=0.0719 \quad \beta_{1}=0.91571 \quad \beta_{2}=-0.01248 \quad \beta_{3}=-1.562
$$

Fig. 7.7 shows a more linear residuals distribution than that for Eq. (7.8) and approves the better performance of the response surface proposed in Eq. (7.9). The p-value for each term in Eq. (7.9) is tabulated in Table 15.

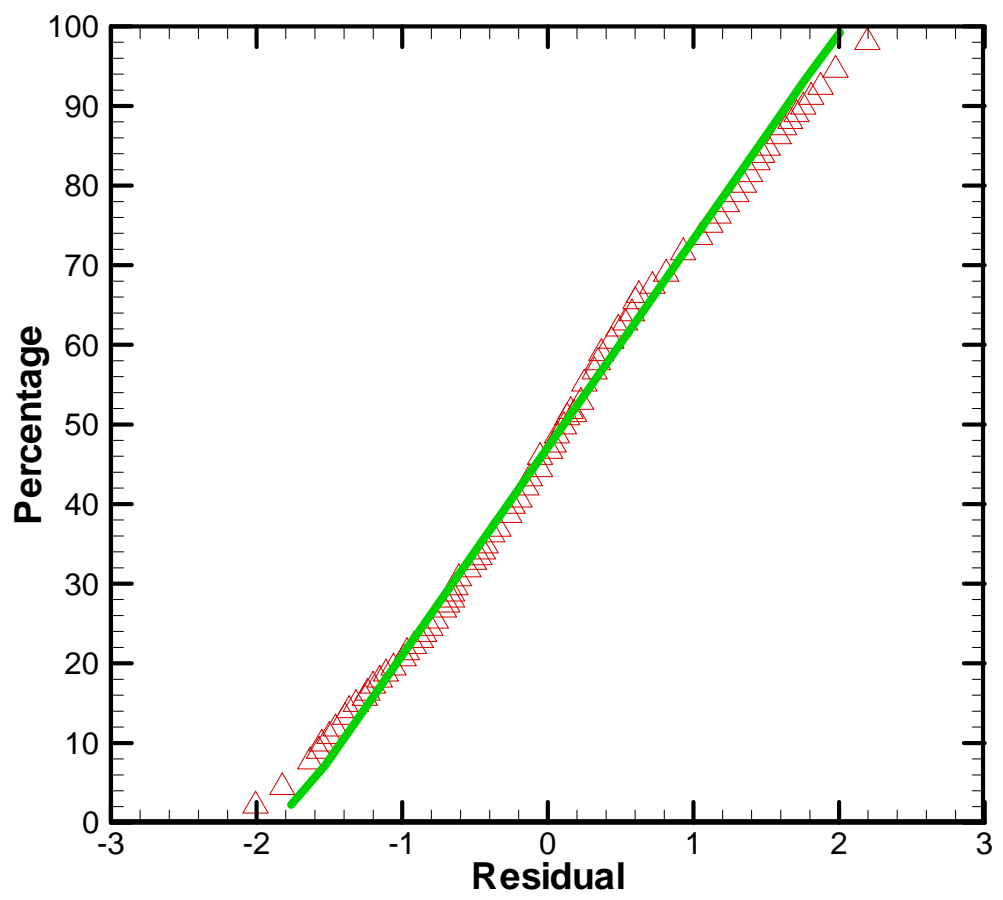

Fig. 7.7: The normal probability of the residuals in Eq. (7.9).

Table 15: P-values for the regression terms in Eq. (7.9).

\begin{tabular}{|l|l|}
\hline Term & p-value \\
\hline Constant & 0.001 \\
\hline$U_{\infty} \cos \theta$ & 0.000 \\
\hline$U_{\infty} \sin \theta$ & 0.000 \\
\hline$k$ & 0.017 \\
\hline
\end{tabular}


The coefficient of determination in a response surface model, denoted by $R^{2}$, indicates the performance of the model in fitting the data and is between 0.0 and 1.0. Theoretically, the closer be $R^{2}$ to the unity, the better would be the estimation of regression. However, in many practical applications of a response surface model, the large value of $R^{2}$ cannot necessarily approve the regression quality. If more variables are added to the model the value of $R^{2}$ increases, regardless of their statistical significance.

As an alternative, the $\operatorname{Radj}^{2}$ is frequently used instead. If unnecessary variables are added to the response model, the value of $\operatorname{Radj}^{2}$ often decrease. Basically, $R^{2}$ indicates how much of the observed variability in the data was accounted for by the model while the $\operatorname{Radj}^{2}$ modifies $R^{2}$ by taking into account the number of covariates or predictors in the model [84, 85]. The formula of the $R_{a d j}{ }^{2}$ is defined earlier in the section 7.1, Eq. (7.7).

For the response surface model proposed in Eq. (7.9) the $R_{a d j}{ }^{2}$ has been measured to be 99.5\% which approves the accuracy and performance of this model to predict the velocity measured by the UA.

The performance of the response surface model proposed in Eq. (7.9) has been compared with the anemometer measurements in two different typical tests which are shown in Fig. 7.8. For the wind speeds and UA rotational velocities examined in the present experiments, the response surface model constructed based on the present experiments has shown a good performance. The present approach while is simple and easy to use, has taken care the shadow regions by a reasonable accuracy. However, it can even be improved to enhance the prediction accuracy in the shadow regions by conducting more experiments in these areas and adding more product terms in the regression.

Note that the empirical relations proposed by previous researches are based on an extensive experimental data bank along with some corrections for shadow effects. Evaluating these correction terms is a try and error process to find the values giving the best fit. One can infer from Fig. 7.8 that the present method, despite not especially been corrected for shadow effect, has a good overall $R^{2}$, indicating a good performance with a simpler process. 

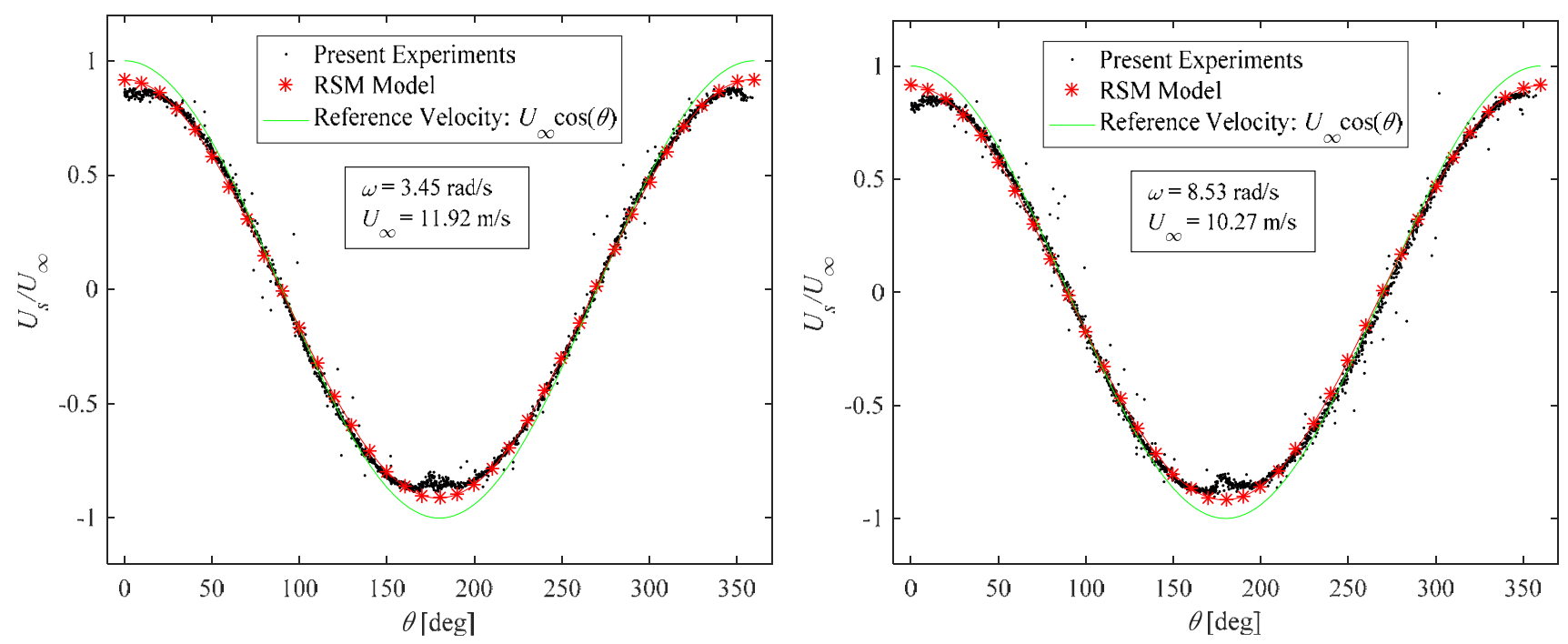

Fig. 7.8: Performance of the present model obtained from RSM with present experiments for two typical spinning tests. 


\subsection{Outlines of the chapter}

An in depth survey has been undertaken to measure the response of an ultrasonic singleaxis anemometer in a wind-tunnel for different wind speeds and anemometer rotational velocities. A simple theoretical model was then proposed based on response surface method to predict the output velocity signal indicated by the anemometer for given wind speed and the anemometer rotational velocity.

Another theoretical model was also proposed based on response surface method to correct the phase shift in the velocity measured by the anemometer for given wind speed and the anemometer rotational velocity. In this model a phase shift was proposed to be a linear function of reduced frequency, time delay between pulse firings, and an exponential parameter including the tangent of the azimuthal angle to account for both the sinusoidal rotation of the UA and the cosine dependency to the expected velocity read by the UA. The output signals read by the sensor, once corrected for this time shift, shows a good agreement with the values actually expected from the UA.

The response surface models proposed in this chapter, can be used to calibrate an ultrasonic transducer in both static and spinning cases. For any other UA of the same type as the one used in this experiment, the regression constants should be determined in an individual calibration process and will evidently be different from those obtained in this thesis. However, for the transducers with different applications, using similar approach, a different regression model can be obtained including the suitable parameters involved in the new application. 


\section{Chapter 8}

\section{Conclusions and future work}

A modified single-axis ultrasonic anemometer was used to measure the wind speed in a subsonic wind tunnel. The experiments were performed for both static and dynamic oscillatory conditions. The objective of the thesis is to find out how a sonic anemometer measures wind speed and direction during the rotational motion, and therefore, propose a calibration process and theoretical model for its velocity measurements during the rotation.

Due to the quality of measurements in the experimental tests, it is reminded that the experiments were performed in IDR/UPM institute which is a member of MEASNET, Measuring Network of Wind Energy Institutes, whose aims are the harmonization of measurements related to wind energy, and the calibration of anemometers among them. The calibration wind-tunnel (S4) in IDR is designed to fulfil the specific requirements of industrial anemometer calibration in accordance with MEASNET. According to MEASNET rules, members also need to have an ISO/IEC17025 accreditation for the specific measurements they perform. The entire calibration process is subject to external audits by ENAC and internal procedures every 12 months in order to ensure the quality of the anemometer calibration process. 


\subsection{Calibration with least square regression}

Firstly, the velocity measured by ultrasonic anemometer in different stationary azimuthal angles of transducers' signal path with wind direction is calibrated with wind-tunnel reference wind speed.

The major source of uncertainty in ultrasonic wind speed measurements is the transducers shadow effect. It is seen that as the misalignment increases, the wake of the upstream transducer is projected out of the ultrasonic path, reducing the distortion that occurs in the measurements.

At the critical angles, $\theta= \pm 5^{\circ}$, the upstream sensor is slightly deviated towards the wind direction so that its cross section faced up with the incoming flow increases, and therefore stronger wakes and vortices are shed into the measurement path. This confirms the role of the aerodynamic design and geometric dimensions in reducing the sensor shadow effect onto the signal path.

From the static tests, the relationship between the wind reference velocities, projections of the incoming wind onto the signal path, and the UA indicated wind speed expressed as a calibration function: $U_{r}=A U_{s}+B$. $A$, and $B$, are the calibration coefficients which depend on the azimuthal angle of the sonic path with respect to the wind direction. The values of $A$ and $B$ are obtained by the least squares regression method.

In the dynamic tests, the anemometer was rotating with different rotational speeds inside the test chamber of the wind tunnel, and different wind speeds were set for each rotating test. The wind speeds measured by UA are synchronized with the instantaneous azimuthal angles measured by angular position sensor at the same time.

In each spinning test, the UA was rotating when the data-recording started. In such conditions, the velocity measurements recorded with a time lag due to the angular position measurements. This time lag or delay is observed in the form of a phase shift or misalignment in the signals measured by the UA. Each test had its own phase shift which was determined and added to the angular position data to correct for yaw misalignment.

The calibration function obtained from the static tests are used to calibrate the UA measurement data in dynamic tests in the form of $U c(t)=A(t) U s(t)+B(t)$. The calibrated measurements in the spinning tests showed a good agreement with the reference velocities. The uncertainty of the calibration process for the spinning tests was found to be about $0.3 \%$. 
It is observed that the measurements were affected mostly with the transducers shadow effect at each instantaneous azimuthal angle of signal path, and the shifting in acoustic pulse trajectory does not affect the wind speed measurements in rotational motion. Therefore, the calibration process for a fixed single-axis ultrasonic anemometer in different stationary angles of signal path with wind direction at a plane can be used for the same anemometer in rotational motion at that plane.

\subsection{Calibration with phase angle}

The signal path of the anemometer was placed at various azimuthal angles with respect to the wind direction. It has been observed that at each angular position, the wake of the transducer imparts a phase angle to the signal direction. A polynomial function was then obtained to calculate this phase shift at each azimuthal angle. On this basis, a calibration model was proposed by calculating the projection of the wind vector onto the signal path in which the azimuthal angle was corrected for the aforementioned phase shift obtained by the polynomial function.

For the single-axis sonic anemometer, the calibration model was obtained in the form of $U_{c}=U_{s} /[\cos (\phi)-\tan (\theta) \times \sin (\phi)]$ where $U_{s}$ is the UA measurements and $\phi$ was obtained with a forth order polynomial function at each azimuthal angle of the signal path with wind direction, $\theta$. The calibrated measurements showed a very good agreement with the reference velocities in both static and spinning tests.

The approach to obtain the calibration model can be used for any other ultrasonic anemometer. Firstly, the signal path of the anemometer should be placed in different stationary azimuthal angles with wind direction (like the directional calibration process), and then the fitting function of the calculated phase angles can be used to construct a calibration model for the same anemometer in the spinning conditions.

\subsection{RSM models}

Remarkable distortion in the measured velocity pattern has been observed which is due to the transducer shadow effect, occurring in the areas where the acoustic path gets parallel to free stream wind direction. The shadow effect in an ultrasonic transducer leads to an underestimation of wind components due to velocity deficits in the wake of the transducer along the acoustic paths. Very limited attempts have been made to model the shadow effect and correct the anemometer indicated velocity in the shadow zones. 
Here, experimental results were conducted to construct a response surface for the velocity indicated by the UA, as the response of interest which is a function of the independent factors: free stream wind velocity, $U_{\infty}$, the angular velocity of the UA, $\omega$, and the instantaneous azimuthal angle, $\theta$, between signal path and inflow direction. Using the wind-tunnel results, a response surface has been fitted to the model and can be used to easily establish a relationship between the UA indicated velocity and the free stream flow velocity.

The RSM model proposed in this thesis has shown to be in a good agreement with the experimental data. While having higher accuracy, the present model is much easier to use than the previously found empirical correlation. The proposed theoretical model can be used for the calibration of such anemometers in rotational conditions and works as well for the stationary condition, by setting the angular velocity in the model to be zero.

In the previously found empirical correlations for calibrating wind speed measured by a spinning ultrasonic anemometer, some constants in the equation should be found from the empirical charts and by try and error to achieve the best fit. However, the proposed method offers a simple polynomial equation in which there is no need to evaluate the coefficients by try and error and can thus be claimed to have less trouble to use while preserving acceptable accuracy.

Besides, for a spinning anemometer, the velocity outputs have a time lag with the angular position outputs which is observed in the form of a phase shift or misalignment in the measurements. Each spinning test has a phase shift which should be calculated and added to the position data for correcting the yaw misalignment. In this thesis, the experimental data were used to construct a theoretical model, based on Response Surface Method, to correct the phase shift for various wind speeds and anemometer rotational velocities. In this model, the phase shift was proposed to be a linear function of reduced frequency, time delay between pulse firings, and an exponential parameter including the tangent of the azimuthal angle to account for both the sinusoidal rotation of the UA and the cosine dependency to the expected velocity read by the UA. The output signals read by the sensor, once corrected for this time shift, shows a good agreement with the values actually expected from the UA. 


\subsection{Calibration of UA in spinner anemometry}

A challenge in wind turbine design is how to achieve an accurate and cheap measurement of the wind that flows into the wind turbine rotor. An accurate knowledge of the incoming wind is important in order to regulate yaw and pitch control for optimized wind power. The spinner anemometry was described as a recently technique in which three single-axis UAs are mounted over the spinner of a wind turbine for measuring the free horizontal and vertical wind speed and direction. Therefore, accurate wind speed measurement, as

performed by a properly calibrated spinner ultrasonic anemometer, would be extremely needed to achieve the maximum performance from energy saving viewpoint.

The calibration process proposed for the phase 1 of the calibration chain in spinner anemometry is that, firstly, the UA used in spinner anemometry is calibrated in various stationary yaw or pitch angles to determine the calibration constants. And then the obtained constants are used to correct the UA measurement data in rotational motion of the signal path. As the wind direction changes in horizontal plane of the UA, the calibration function obtained from the stationary yaw angles will be used for correcting the measurements. As the wind direction changes in vertical plane of the UA, the calibration function obtained from the stationary pitch angles will be used for correcting the measurements. This would be done by the calibration processes proposed in this thesis either with least square regression method or phase angle method.

Additionally, the RSM approach proposed in this thesis can be employed, not only in phase 1 of the calibration chain, in which a calibration model for the isolated sensor is to be determined, but also in more general cases such as a UA sensor installed on a spinner hub. The latter, necessitates that new parameters depending on the nature of the problem under consideration come into play and a new regression model would be achieved. 


\subsection{Future work}

In this thesis, a calibration process for wind speed and direction measurements with a modified single-axis ultrasonic anemometer has been performed in static and spinning conditions. A similar calibration process for the sonic anemometer type used in spinner anemometry would suggest to be performed in the future.

In this dissertation, the results obtained from two typical static tests have been compared with CFD simulations, however, the results in spinning tests would suggest to be compared with CFD as a future work.

Additionally, the theoretical model obtained by RSM approach to predict the wind speed measurements can be compared with CFD simulations for the sonic anemometer of the same type in both static and spinning conditions.

A deeper survey on ultrasonic velocity measurements specifically in the transducer shadow regions would suggest to be performed with the help of response surface methodology.

The author suggests that once the independent parameters responsible for the UA reading are properly identified, the RSM can be a powerful tool to obtain the calibration equation. For any applications, including spinner anemometry, there are some new variables or combination of several variables which must be added to the regression equation to specifically calibrate the UA sensor under consideration. The starting point in RSM approach is to identify the independent factors that affect the response. Appropriate independent variables are thus needed to be chosen for constructing the RSM model which can cover the entire calibration phases of spinner anemometry. 


\section{References}

[1] Suomi VE, (1957) Sonic Anemometer, In Exploring the Atmosphere's First Mile. Instrument, Vol. 1, Pergamon, New York, pp. 256-266

[2] Kaimal JC, (1979) Sonic anemometer measurement of atmospheric turbulence, Proceedings of the Dynamic Flow Conference, Skovlunde, Denmark (DISA Electronic A/S), pp. 551-565

[3] Kaimal JC, Wyndgaard JC, (1990) The Kansas and Minnesota experiments. Boundary Layer Meteorol. 50: 31-47

[4] Cuerva A, Sanz-Andres A, (2000) On sonic anemometer measurement theory. Journal of Wind Engineering and Industrial Aerodynamics 88: 25-55

[5] Pedersen TF, (2008) Method and apparatus to determine the wind speed and direction experienced by a wind turbine. United States Patent, Patent No.: US 7,347,668 B2

[6] Frandsen S, (2006) Nacelle anemometry. Proceedings of EWEA 2006, Bruxelles

[7] Zahle F, Srensen NN., (2011) Characterization of the unsteady flow in the nacelle region of a modern wind turbine. Wind Energy 14: 271-283

[8] Pedersen TF, Demurtas G, Sommer A, Hojstrup J, (2014) Measurement of rotor centre flow direction and turbulence in wind farm environment. Journal of Physics, Conference series: 524-012167

[9] Kristensen L, (2002) Can a cup anemometer under speed. Boundary-Layer Meteorology 103:163-172

[10] Pedersen TF, (2004) On wind turbine power performance measurements at inclined airflow. Journal of Wind Energy 7:163-176

[11] Jorgen H, (2014) Increased energy production by optimization of yaw control. VGBCongress, Power Plant, Hamburg, Germany

[12] Pedersen TF, Demurtas G, and Zahle F, (2015) Calibration of a spinner anemometer for yaw misalignment measurements. Wind Energy, 18(11): 1933-1952

[13] Demurtas G, Pedersen TF, and Zahle F, (2016) Calibration of a spinner anemometer for wind speed measurements. Wind Energy, 19(11): 2003-2021

[14] Standard: IEC 61400-12-2: (2012) Power Performance of Electricity Producing Wind Turbines Based on Nacelle Anemometry. $1^{\text {st }}$ Edition, International Electro Technical Commission: Geneva, Switzerland

[15] Report from ROMO Wind A/S (2015), Review of the spinner anemometer from ROMO Wind iSpin. Report No.: 113605-DKAR-R-0-1, Rev. 3

[16] Everest FA, (2001) The master handbook of acoustics. $4^{\text {th }}$ edition, McGraw-Hill, New York

[17] Bruneau M, Scelo T, (2006) Fundamentals of acoustics. ISTE Ltd, London

[18] Auld BA, (1990) Acoustic Fields and Waves in Solids. $2^{\text {nd }}$ Edition, Vol. 1 \& 2, Krieger Publishing, Florida

[19] Krautkramer J, Krautkramer H, (1990) Ultrasonic Testing of Materials. $4^{\text {th }}$ Revised Edition, Springer-Verlag, Berlin 
[20] Jewett JW, Serway RA, (2004) Physics for scientists and engineers. 6th edition, Thomson, Toronto

[21] Cheeke J, David N, (2002) Bulk waves in fluids, Principles and applications of ultrasonics. CRC, Boca Raton, 39-58

[22] Colin H Hasan, Fundamentals of acoustics. Department of mechanical engineering, University of Adelaide, South Australia

[23] Kuttruff H, (1991) Ultrasonics - fundamentals and applications. London, Elsevier Applied Science

[24] Elmore WC, Heald MA (1985) Physics of Waves, Dover Publications. New York

[25] Thurston RN, Pierce AD, (1999) Ultrasonic Instruments and Devices I \& II, Academic Press, San Diego

[26] Arnau A, (2004) Piezoelectric Transducers and Applications. Springer-Verlag, Berlin

[27] Carlin B (1960) Ultrasonics, $2^{\text {nd }}$ edition. McGraw-Hill, New York

[28] Lempriere BM, (2002) Ultrasound and Elastic Waves: Frequently Asked Questions. Academic Prerss, San Diego

[29] Suslick, KS, (1998) Kirk-Othmer Encyclopedia of Chemical Technology. $4^{\text {th }}$ edition, J. Wiley \& Sons: New York, 1998, vol. 26, 517-541

[30] Edwards J, Frequency (2006) Domain Theory and Applications. BEng. (Hons), CEng., MIEE, MIEEE, UK

[31] Jayanthy AK, Sujatha N, Ramasubba M, (2011) Measuring blood flow: techniques and applications. Journal of IJRRAS: 6(2)

[32] Arlotto P, Grimaldi M, Naeck R, Ginoux JM, (2014) An ultrasonic contactless sensor for breathing monitoring. Journal of Sensor: 14(8): 15371-15386

[33] Curry GB, White DN, (1978) Color-coded ultrasonic differential velocity arterial scanner (echo flow). Ultrasonic in Medicine and Biology, vol. 4, p. 27

[34] Kasai C, Namekawa K, Koyama A, Omoto R, (1985) Real-time two-dimensional blood flow imaging using an autocorrelation technique. IEEE Trans. Son. And Ultrason., vol. 32, pp. 458-463

[35] Strohrmann G, (1994) atp-Marktanalyse Durchfluß- und Mengenmeßtechnik (Teil 2), Atp, 36, 9-29

[36] Williams J, (2003) Introduction to Fluid mechanics. PWS-Kent Publishing Company, Boston

[37] Neil Colin, (2002) Optimization of an ultrasonic flow meter based on experimental and numerical investigation of flow and ultrasound propagation. Doctoral Thesis in Mechanical and Manufacturing Engineering, University of New South Wales

[38] Swengel RC, Hess WB, Waldorf SK, (1955) Principles and Application of the Ultrasonic flowmeter. Winter General Meeting American Institute of Electrical Engineers, Power Apparatus and Systems, No. 17, PP 112-118.

[39] Swengel RC, Hess WB, Waldorf SK, (1954) Demonstration of the Principles of the Ultrasonic Flowmeter. American Institute of Electrical Engineers, Conference Paper, AIEE Middle Eastern District Meeting, Electrical Engineering, Vol. 73, No. 12, PP 1082-1084 
[40] Shangari B, Meleschi, (2004) Ultrasonic technique in determination of GridGenerated turbulent flow characteristics and caustic formation. Master thesis in Mech. Dept. of Worcester Polytechnic Institute

[41] Walker IJ, (2004) physical and logistical consideration of using ultrasonic anemometers in Aeolian sediment transport research. Journal of Geomorphology 68: 57-76

[42] Cuerva A, Benzdenejnykh N, Parales JM, Sanz-Andres A, (1998) Assessment of performances of ultrasonic anemometers as one step ahead in wind measurements of energy production of a wind turbine. Universidad Politécnica de Madrid

[43] Mortarini L, Ferrero E, Richiardone R, Falabino S, Anfossi D, Trini Castelli S, and Carretto E, (2009) Assessment of dispersion parameterizations through wind data measured by three sonic anemometers in a urban canopy. Adv. Sci. Res., 3, 91-98, 2009

[44] Rodenhiser RJ, Durgin WW, Johari H, (2007) Ultrasonic method for aircraft wake vortex detection. Journal of Aircraft, Vol. 44, No. 3

[45] Johari H, Durgin W, (1998) Direct Measurement of Circulation Using Ultrasound. Experiments in Fluids, Vol. 25, Nos. 5-6, pp. 445-454

[46] Mandard E, Kouame D, Battault R, Remenieras JP, Patat F, (2005) Transit time ultrasonic flowmeter: velocity profile estimation. IEEE Ultrasonic Symposium, PP 763-766

[47] Hans V, Windorfer H, (2003) Comparison of pressure and ultrasound measurements in vortex flow meters. Measurement: 121-133

[48] Franchini S, Sanz-Andre's A, Cuerva A (2007) Effect of the pulse trajectory on ultrasonic fluid velocity measurement. Journal of Exp Fluids, Vol. 43: 969-978

[49] Franchini S, (2006) Source of uncertainty in sonic anemometry. PhD dissertation, ETSI Aeronáuticos, Universidad Politécnica de Madrid

[50] Miller DO, Tong C, Wyngaard JC, (1999) The effects of probe-induced flow distortion on velocity covariance: Field observations. Bound.-Layer Meteor., 91, 483-493

[51] Wieser A, Fiedler F, Corsemeier U, (2001) The influence of the sensor design on wind measurements with sonic anemometer systems. J. Atmos. Oceanic Technol., 18, PP 1585-1608

[52] Wyngaard JC, (1981) The effect of probe-induced flow distortion on atmospheric turbulence measurements. J. Appl. Meteor., 20, 784-794

[53] Wyngaard JC, Zhang SF, (1985) Transducer Shadow Effects on Turbulence Spectra Measured by Sonic Anemometers. Journal of Atmospheric and Oceanic Technology 2: PP 548-558

[54] Cuerva A., Pedersen T.F., Luengo S., Pastor J.A., Sanz-Andrés A., and Franchini S., 2008: A theoretical model on spinner anemometry based on ultrasonic paths, European Wind Energy Conference and Exhibition 2008. ISBN: 978-1-61567-1151, Brussels, Belgium 31 March - 3 April 2008. 
[55] Mortensen NG, (1994) Flow-Response Characteristics of the Kaijo Denki OmniDirectional Sonic Anemometer (TR-61B). RISØ-R-704(EN), DTU, Roskilde, Denmark

[56] Kaimal JC, Wyngaard JC, Haugen D, (1968) Deriving power spectra from a threecomponent sonic anemometer. J Appl Meteor, 7: PP 827-837

[57] Silverman B, (1968) The effect of spatial averaging on spectrum estimation. J Appl Meteor, 7: PP 168-172

[58] Kristensen L, Fitzjarrald D, (1984) The effect of line averaging on scalar flux measurements with a sonic anemometer near the surface. J Atmos Ocean Technol, 1: PP 138-146

[59] Cuerva A, Sanz-Andres A, Navarro FJ, (2003) On multiple-path sonic anemometer measurement theory. Exp. in Fluids, 34: PP 345-357

[60] Pedersen TF, Sorensen NN, Vita L, Enevoldsen P, (2008) Optimization of wind turbine operation by use of spinner anemometer. RIS $\varnothing-R-1654(E N)$, DTU, Roskilde, Denmark

[61] Pedersen TF, Sorensen NN, Enevoldsen P, Egedal P, (2007) Spinner Anemometry an innovative wind measurement concept. EWEC2007 Conference, 7-10 May, Milan, Italy

[62] Pedersen TF, Sorensen NN, Enevoldsen P, (2007) Aerodynamics and characteristics of a spinner anemometer. Journal of Physics, Conference series: 75-012018

[63] Pedersen TF, Vita L, Sorensen NN, Enevoldsen P, (2008) Operational experiences with a spinner anemometer on a MW Size Wind Turbine. Conference of EWEC2008, 31 March - 3 April, Bruxelles

[64] Sorensen NN, Pedersen TF, (2007) CFD computation around wind turbine spinner and nacelle. Risø-I-2579 (EN)

[65] Tammelin B, Heimo A, Leroy M, (2000) "Ice-free sensors - The EUMETNET SWS II project", Finnish Meteorological Institute, Helsinki

[66] Demurtas G, Pedersen TF, (2014) Summary of the steps involved in the calibration of a spinner anemometer. DTU Wind Energy Report I-0364, Roskilde

[67] Jorgen H, (2013) Power curve measurements using the ROMO Wind Spinner Anemometer. ROMOWind Company, Wind Farm Owner's Advocate and Contract Developer

[68] Smith MJ, (2014) Statistical Analysis Handbook. A comprehensive handbook of statistical concepts, techniques and software tools, (Winchelsea: Winchelsea Press)

[69] Keane AJ, (2003) Wing Optimization Using Design of Experiment, Response Surface, and Data Fusion Methods. Journal of Aircraft, Vol. 40, No. 4, PP. 741-750

[70] Bettinger RA, Black JT, and Agte JS, (2015) Design of Experiment Approach to Atmospheric Skip Entry Maneuver Optimization, Journal of Spacecraft and Rockets, Vol. 52, No. 3, PP. 813-826

[71] Box GEP, and Draper NR, 2007: Response Surfaces, Mixtures, and Ridge Analyses, $2^{\text {nd }}$ Edition, John Wiley and Sons, New York

[72] Montgomery DC, (2005) Design and Analysis of Experiments: Response surface method and designs. New Jersey: John Wiley and Sons, Inc. 
[73] Khuri AI, (2006) Response Surface Methodology and Related Topics. Singapore, World Scientific Publications. ISBN: 978-981-4479-58-5

[74] Myers RH, Montgomery DC, and Anderson-Cook CM, (2016) Response surface methodology: process and product optimization using designed experiments. 4th edition, John Wiley and Sons Inc. ISBN: 978-1-118-91601-8

[75] Bradley N, (2007) The Response Surface Methodology, MS Thesis, Indiana University at South Bend

[76] Box GEP, and Wilson KB, (1951) On the Experimental Attainment of Optimum Conditions. Journal of the Royal Statistical Society, Series B (Methodological) Vol. 13, No. 1, PP. 1-45

[77] DeLoach R, (1998) Applications of Modern Experiment Design to Wind-tunnel Testing at NACA Langley Research Center. AIAA 98-0713, 36th AIAA Aerospace Sciences Meeting and Exhibit, Reno, NV

[78] DeLoach R, (2000) The Modern Design of Experiments: A Technical and Marketing Framework. AIAA 2000-2691, 21st AIAA Advanced Measurement Technologies and Ground Testing Conference, 19-21 June 2000, Denver, Co.

[79] DeLoach R, and Micol JR, (2010) Analysis of Wind-tunnel Polar Replicates Using the Modern Design of Experiments (Invited). AIAA 2010-4927, 27th AIAA Aerodynamic Measurement Technology and Ground Testing Conference 28 June 1 July, Chicago, Illinois

[80] Cuerva A, and Sanz-Andrés A, (2003) Sonic anemometry of planetary atmospheres. JOURNAL OF GEOPHYSICAL RESEARCH, VOL. 108, NO. E4, 5029

[81] Stephens MA, (1974) EDF Statistics for Goodness of Fit and Some Comparisons. Journal of the American Statistical Association. Vol. 69, No. 347, PP 730-737

[82] Kutner MH, Nachtsheim CJ, and Neter J, (2004) Applied Linear Regression Models, $4^{\text {th }}$ ed. McGraw-Hill Irwin

[83] Longnecker MT, and Ott RL, (2004) A First Course in Statistical Methods. Thomson Brooks/Cole. p. 615

[84] Haber A, and Runyun RP, (1977) General statistics. $3^{\text {rd }}$ edition, Reading, MA: Addision-Wesley

[85] Mason RL, Gunst RF, and Hess JL, (2003) Statistical Design and Analysis of Experiments: With Applications to Engineering and Science. $2^{\text {nd }}$ Edition, John Wiley \& Sons Publications

[86] Milliken GA, and Johnson DE, (2009) Analysis of Messy Data Volume 1: Designed Experiments. $2^{\text {nd }}$ Edition, CRC Press., Taylor \& Francis Group, LLC. 


\section{Appendix A}

\section{Specifications of belt and pulley}

\section{A.1. Belt and Pulley Pitch}

Belt pitch, $P$, is defined as the distance between the centrelines of two adjacent teeth and is measured at the belt pitch line as shown in the Fig. A.1. The belt pitch line is identical to the neutral bending axis of the belt and coincides with the centre line of the cords. Pulley pitch is measured on the pitch circle and is defined as the arc length between the centrelines of two adjacent pulley grooves, Fig. A.1. (a) and Fig. A.1. (b). The pitch circle coincides with the pitch line of the belt while wrapped around the pulley. In timing belt drives the pulley pitch diameter, $d$, is larger than the pulley outside diameter, $d_{0}$. The pulley pitch diameter is given by:

$$
d=\frac{P \times Z_{p}}{\pi}
$$

where $P$ is the nominal pitch and $Z_{p}$ the number of pulley teeth.

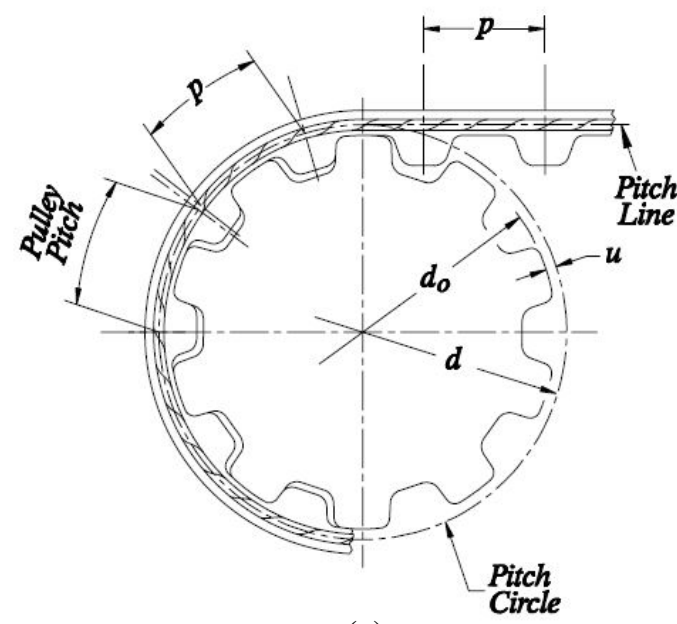

(a)

Belt and pulley mesh for inch series and metric

T-series, HTD and STD series geometry.

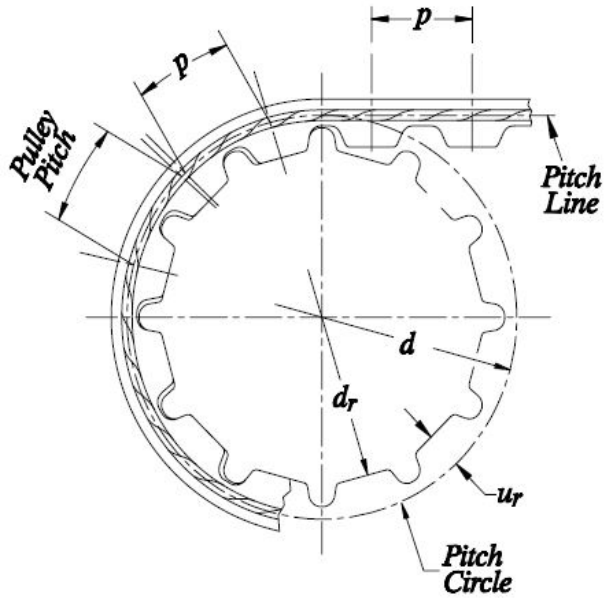

(b) series geometry.

Fig. A.1: Pitch definition in belt and pulley. 
The radial distance between pitch diameter and pulley outer diameter is called 'pitch deferential', $u$, and has a standard value for a given belt section of inch pitch and metric $T$-series belts. The pulley outside diameter can be expressed by:

$$
d_{0}=d-2 u=\frac{P \times Z_{p}}{\pi}-2 u
$$

\section{A.2. Belt Length and Centre Distance}

Belt length, $L$, is measured along the pitch line and must equal a whole number of the belt pitches or belt teeth, $Z$ :

$$
L=P \times Z_{b}
$$

Most linear actuators and conveyors are designed with two equal diameter pulleys. The relationship between belt length ' $L$ ', centre distance ' $C$ ', and pitch diameter ' $d$ ' is given by:

$$
L=2 \times C+\pi \times d
$$

For drives with two unequal pulley diameters as shown in the Fig. A.2, the following relationships can be given:

Angle of wrap $\left(\theta_{1}\right)$ around the small pulley:

$$
\theta_{1}=2 \times \arccos \left(\frac{d_{2}-d_{1}}{2 \times C}\right)
$$

where, $d_{1}$ and $d_{2}$ are the pitch diameters of the small and the large pulley, respectively. Therefore, the angle of wrap $\left(\theta_{2}\right)$, around the large pulley is calculated as: $\theta_{2}=2 \times \pi$ - $\theta_{1}$ Span length $\left(L_{s}\right)$ is calculated as:

$$
L_{s}=C \times \operatorname{Sin}\left(\frac{\theta_{1}}{2}\right)
$$

Belt length $(L)$ is calculated as:

$$
L=2 \times C \times \operatorname{Sin}\left(\frac{\theta_{1}}{2}\right)+\theta_{1} \times \frac{d_{1}}{2}+\left(2 \pi-\theta_{1}\right) \times \frac{d_{2}}{2}
$$

Since angle of wrap $\left(\theta_{1}\right)$, is a function of the center distance $(C)$, and does not have a closed form solution for $(C)$. It can be solved using any of available numerical methods. 


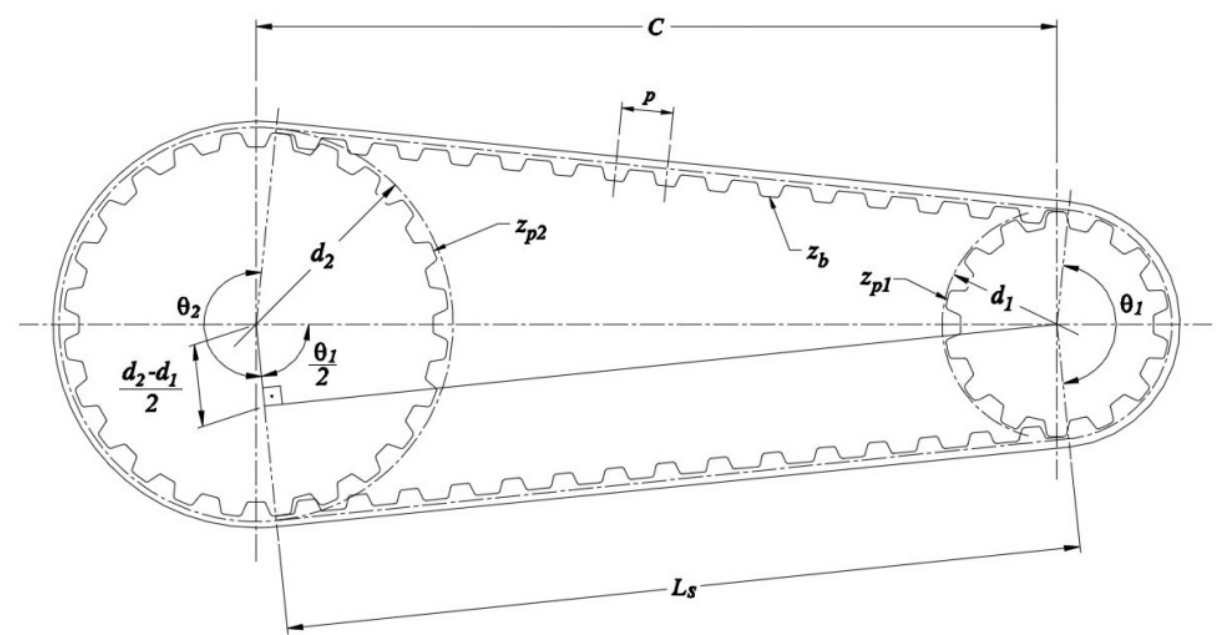

Fig. A.2: Belt drive with unequal pulley diameters.

The approximate belt circum-length $L_{p}{ }^{\prime}$ is determined in terms of temporary inter shaft distance $C^{\prime}$, diameter of large pulley $d_{2}$ and diameter of small pulley $d_{1}$, as below:

$$
L_{P}^{\prime}=2 C^{\prime}+\frac{\pi\left(d_{2}+d_{1}\right)}{2}+\frac{\left(d_{2}-d_{1}\right)^{2}}{4 C^{\prime}}
$$

Therefore, the belt circum-length $\left(L_{p}\right)$, can be obtained from the following procedure:

1. Guess the distance between two shafts, $C^{\prime}$ Temporary Inter-shaft distance

2. Obtain the $\left(L_{p}\right)$ approximate belt circum-length from the Eq. (A.8)

3. Choose the nearest value to approximated belt circum-length from the catalogue $\left(L_{p}\right)$

4. Calculate the correct inter-shaft distance using the following formula:

$$
b=2 L_{P}-\pi\left(d_{2}+d_{1}\right) ; C=\frac{b+\sqrt{b^{2}-8\left(d_{2}-d_{1}\right)^{2}}}{8}
$$

The inter-shaft distance $(C)$ is the key factor that is considered to choose the belt from the catalogues as it is used in section 5.4.4 to choose the transmission belt. 


\section{Appendix B}

\section{CFD Simulations with Ansys-Fluent}

In the static experimental tests, the ultrasonic anemometer was placed inside the wind tunnel test section in different stationary azimuthal angles between signal path and inflow direction, $\theta$. In order to compare experimental data and computational results, the $3 \mathrm{D}$ model of the ultrasonic anemometer is simulated by Ansys-Fluent with the same conditions as applied in the experiments. Hence, the CFD model is solved, using k-epsilon method, for some typical static azimuthal angles and wind speeds as applied in the experiments. Here, the results from the experimental tests in stationary azimuthal angles of $0^{\circ}$ and $60^{\circ}$ are compared with the CFD simulations.

\section{B.1. CFD simulations for the azimuthal angle of $0^{\circ}$}

Firstly, the UA geometry is created and five different meshing volumes are generated around the anemometer's signal path. The closest one to the transducers is the finest one and the farther one is the courser one, as shown in the Fig. B.1.

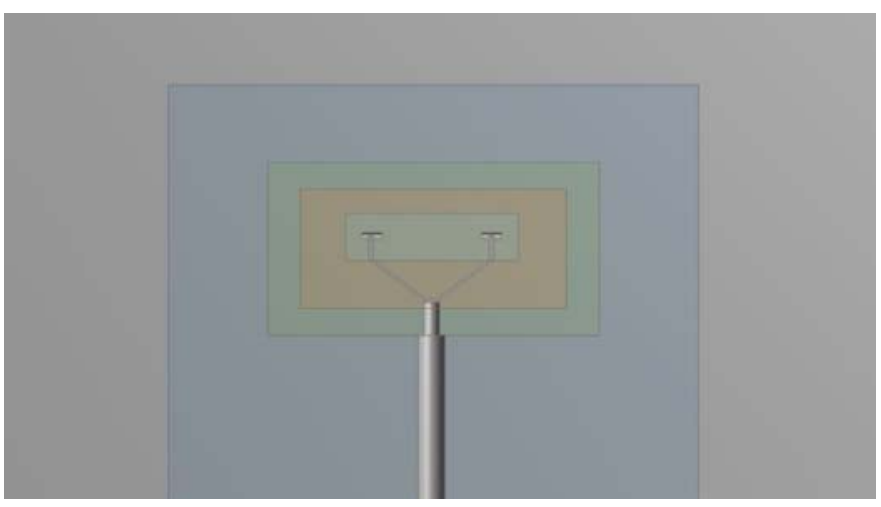

Fig. B.1: UA model and meshing volumes for the azimuthal angle of $0^{\circ}$.

Fig. B.2 illustrates the mesh grid of the test section, and specifically the area around the sonic transducers. It is seen that the mesh grid is finer in the flow field region between two transducers in order to increase the accuracy of CFD simulations. 


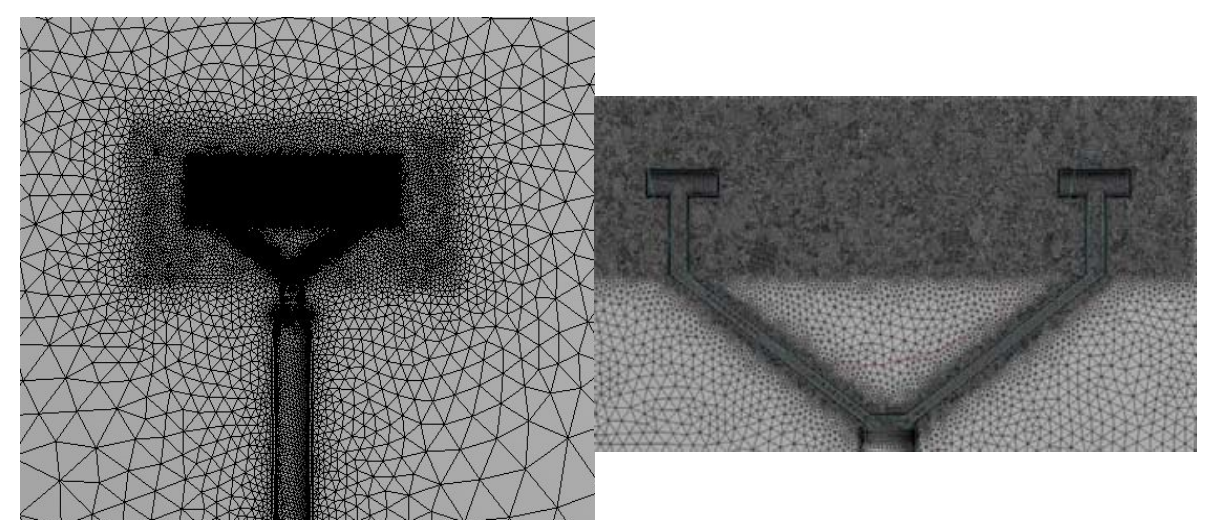

Fig. B.2: Mesh grid of the test section and around the sonic transducers.

The CFD model is solved for an inlet air speed of $13.8 \mathrm{~m} / \mathrm{s}$ which is the same as one of the wind-tunnel wind speeds applied in a static test. In that case, the wind speed was measured by pitot tube as $13.8 \mathrm{~m} / \mathrm{s}$ and the velocity measured by UA was $11.9 \mathrm{~m} / \mathrm{s}$. Fig. B.3 shows the results of the CFD simulations for the flow field velocities of the test section where the flow is coming from the left side.

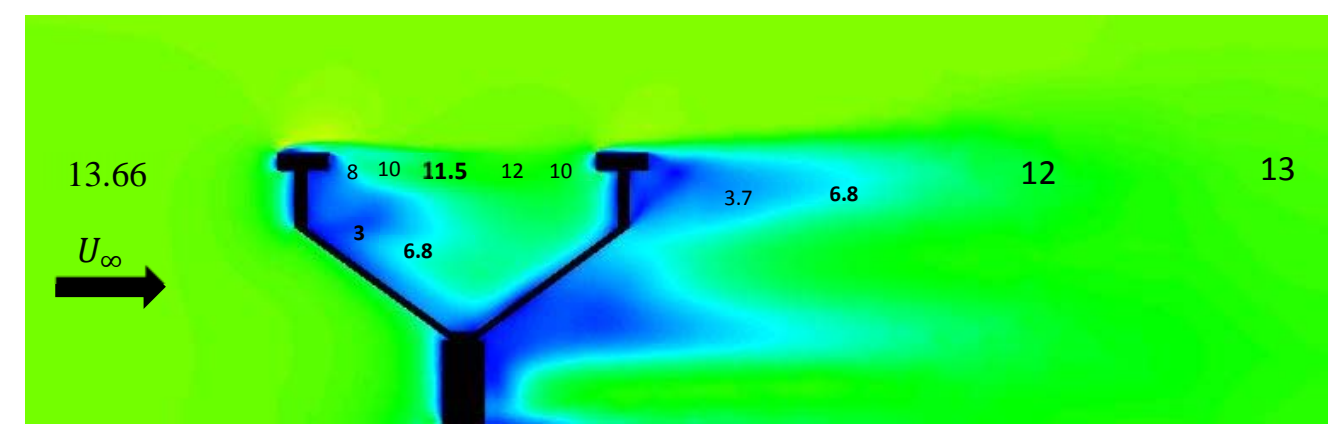

Fig. B.3: Contour of the velocity magnitude for a typical test with inlet velocity of 13.8 $\mathrm{m} / \mathrm{s}$ at the azimuthal angle of $0^{\circ}$.

As it is seen from the figure, the far field wind speed and the inlet velocity are equals, however, they are different in the regions under the wake effect of transducers and supports. The velocity in the middle of signal path between two transducers is computed $11.5 \mathrm{~m} / \mathrm{s}$ by Fluent which is approximately equal to the velocity measured by UA in the experiment, $11.9 \mathrm{~m} / \mathrm{s}$.

Additionally, at this azimuthal angle, $0^{\circ}$, the CFD model is calculated for the inlet air velocity of $4.08 \mathrm{~m} / \mathrm{s}$ (equals to the lowest wind speed applied in the experimental test). Due to the experimental data, the velocity measured by UA was $3.49 \mathrm{~m} / \mathrm{s}$. The velocity measured by CFD in the midpoint of the signal path is $3.45 \mathrm{~m}$, as shown in the Fig. B.4. 


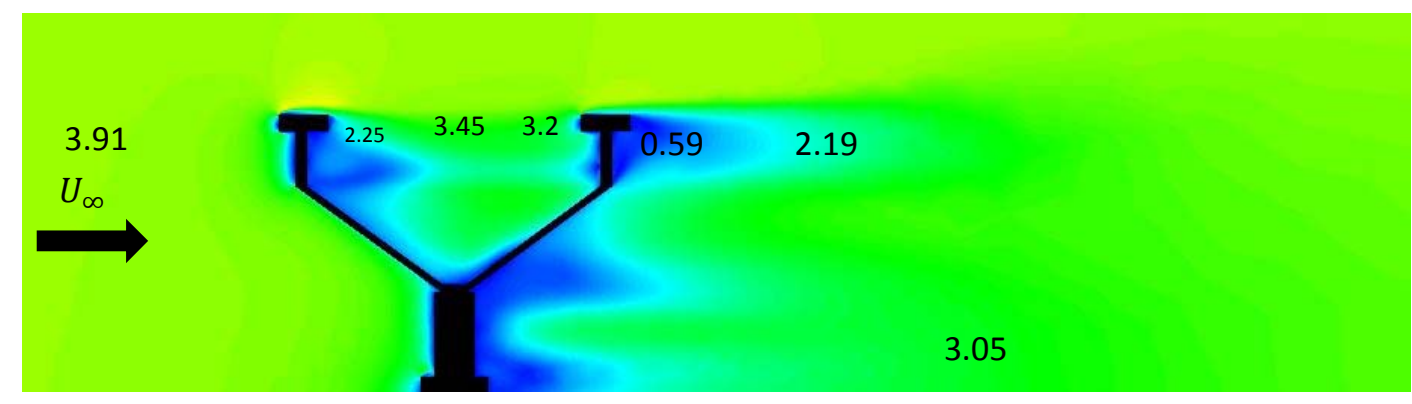

Fig. B.4: Contour of the velocity magnitude for a typical test with inlet velocity of 4.08 $\mathrm{m} / \mathrm{s}$ at the azimuthal angle of $0^{\circ}$.

\section{B.2. CFD simulations for the azimuthal angle of $60^{\circ}$}

It is shown in the Fig. B.5, the UA geometry installed in the test section at the azimuthal angle of $60^{\circ}$ with the meshing volumes around its signal path.

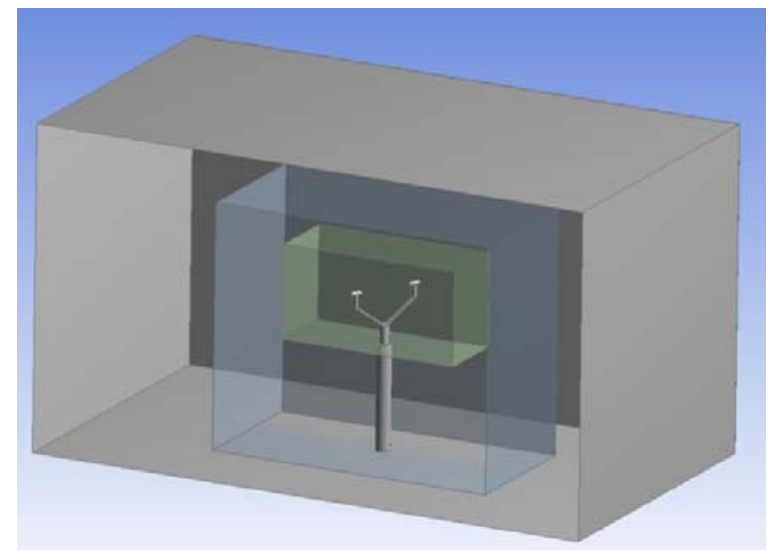

Fig. B.5: UA model and meshing volumes for the azimuthal angle of $60^{\circ}$.

The CFD model is solved for the inlet velocity of $13.8 \mathrm{~m} / \mathrm{s}$. The contour of the velocity magnitude at the horizontal plane crossing the anemometer's signal path is illustrated in the Fig. B.6. It is seen that the flow field in the downstream of the transducers is distorted by the wake of the transducers. However, there is no wake in the midpoint of the signal path between two transducers, and the velocity is computed as $13.44 \mathrm{~m} / \mathrm{s}$ at this region. From the experimental result, the velocity measured by UA was $6.88 \mathrm{~m} / \mathrm{s}$ at this azimuthal angle. If the UA experimental measurement is divided by cosines of $60^{\circ}$, the uniform velocity can be calculated as $13.76 \mathrm{~m} / \mathrm{s}$, which is quite similar to the velocity computed 
by CFD simulation in the midpoint of the signal path between transducers. This approves that as the misalignment increases, the less wake and vortices are shed into the anemometer signal path and the measurement are not affected by the transducers wake.

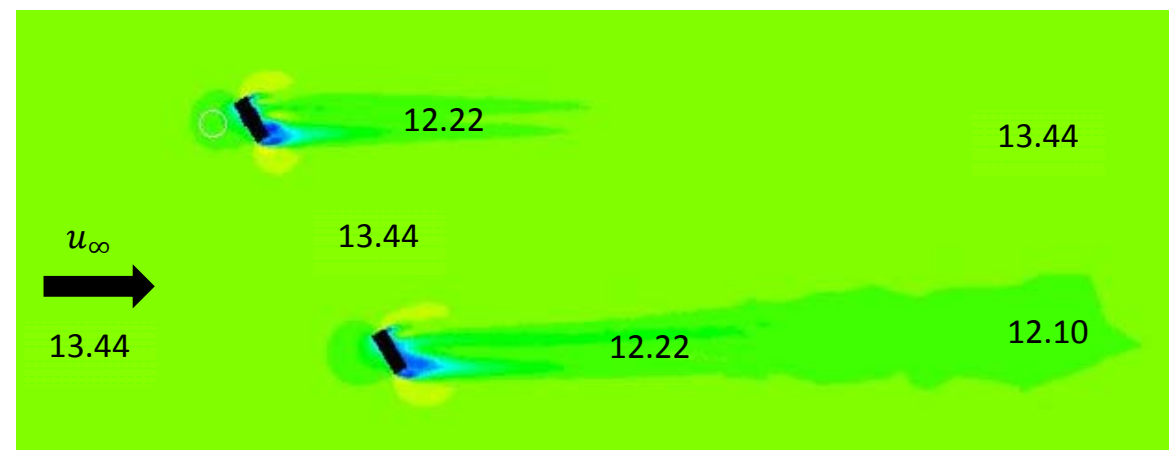

Fig. B.6: Contour of the velocity magnitude for a typical test with inlet velocity of 13.8 $\mathrm{m} / \mathrm{s}$ at the azimuthal angle of $60^{\circ}$.

\section{B.3. Outlines of the appendix}

It is observed that the computational and experimental results validate each other by having very similar measurements for the velocity magnitude in the midpoint of the transducers' signal path. The CFD solved the model while the only parameter that affects the flow field velocity is the presence of UA model inside the simulated test chamber. From the experimental tests, it was concluded that the UA wind speed measurements affected mostly by the transducer head distortion, as discussed in chapter 6. Also, the CFD results in this appendix have shown that the main source of uncertainty in the UA wind speed measurements is the transducer wake and distortion into the signal path. 